\title{
AN OPTICAL SPECTROSCOPIC STUDY OF T TAURI STARS. I. PHOTOSPHERIC PROPERTIES
}

\author{
Gregory J. HercZeg ${ }^{1,2,3,4}$ And LynNe A. Hillenbrand ${ }^{2}$ \\ ${ }^{1}$ Kavli Institute for Astronomy and Astrophysics, Peking University, Yi He Yuan Lu 5, Haidian Qu, Beijing 100871, China \\ ${ }^{2}$ Caltech, MC105-24, 1200 East California Boulevard, Pasadena, CA 91125, USA \\ ${ }^{3}$ Max-Planck-Institut für extraterrestrische Physik, Postfach 1312, D-85741 Garching, Germany \\ Received 2013 August 26; accepted 2014 March 4; published 2014 April 22
}

\begin{abstract}
Estimates of the mass and age of young stars from their location in the H-R diagram are limited by not only the typical observational uncertainties that apply to field stars, but also by large systematic uncertainties related to circumstellar phenomena. In this paper, we analyze flux-calibrated optical spectra to measure accurate spectral types and extinctions of 281 nearby T Tauri stars (TTSs). The primary advances in this paper are (1) the incorporation of a simplistic accretion continuum in optical spectral type and extinction measurements calculated over the full optical wavelength range and (2) the uniform analysis of a large sample of stars, many of which are well known and can serve as benchmarks. Comparisons between the non-accreting TTS photospheric templates and stellar photosphere models are used to derive conversions from spectral type to temperature. Differences between spectral types can be subtle and difficult to discern, especially when accounting for accretion and extinction. The spectral types measured here are mostly consistent with spectral types measured over the past decade. However, our new spectral types are one to two subclasses later than literature spectral types for the original members of the TW Hya Association (TWA) and are discrepant with literature values for some well-known members of the Taurus Molecular Cloud. Our extinction measurements are consistent with other optical extinction measurements but are typically 1 mag lower than near-IR measurements, likely the result of methodological differences and the presence of near-IR excesses in most CTTSs. As an illustration of the impact of accretion, spectral type, and extinction uncertainties on the H-R diagrams of young clusters, we find that the resulting luminosity spread of stars in the TWA is $15 \%-30 \%$. The luminosity spread in the TWA and previously measured for binary stars in Taurus suggests that for a majority of stars, protostellar accretion rates are not large enough to significantly alter the subsequent evolution.
\end{abstract}

Key words: stars: low-mass - stars: pre-main sequence

Online-only material: color figures, machine-readable table

\section{INTRODUCTION}

Classical T Tauri stars (CTTSs) are the adolescents of stellar evolution. The star is near the end of its growth and almost fully formed, with a remnant disk and ongoing accretion. The accretion/disk phase typically lasts $\sim 2-5 \mathrm{Myr}$, though some stars take as long as $10 \mathrm{Myr}$ before losing their disks and emerging toward maturity. Strong magnetic activity leads to pimply spots on the stellar surfaces. Some T Tauri stars (TTSs) are still hidden inside their disks, not yet ready to emerge. Manic mood swings change the appearance of the star and are often explained with stochastic accretion. Depression has been seen in light curves on timescales of days to years. Sometimes every CTTS seems as uniquely precious as a snowflake.

TTS properties were systematically characterized in seminal papers by, e.g., Cohen \& Kuhi (1979), Herbig \& Bell (1988), Basri \& Batalha (1990), Valenti et al. (1993), Hartigan et al. (1995), Kenyon \& Hartmann (1995), Gullbring et al. (1998). In the last decade, dedicated optical and IR searches revealed thousands of young stars, typically confirmed with spectral typing (e.g., Hillenbrand 1997; Briceno et al. 2002; Luhman 2004; Rebull et al. 2010). However, significant differences in extinction and accretion properties between different papers and methods has led to confusion in the properties of even the closest and best studied samples of young stars.

Some of this confusion is exacerbated by stochastic and rotation variability of TTSs. While manic and depressive periods

\footnotetext{
4 Visiting Astronomer, LERMA, Observatoire de Paris, ENS, UPMC, UCP, CNRS, 61 avenue de l'Observatoire, F-75014 Paris, France.
}

provide fascinating diagnostics of the stellar environment and star-disk interactions, they also pose significant problems for assessing the stellar properties and evolution of the star/disk system. How disk mass, structure and accretion rate change with age and mass requires accurate spectral typing and luminosity measurements (e.g., Furlan et al. 2006; Sicilia-Aguilar et al. 2010; Oliveira et al. 2013; Andrews et al. 2013). While median cluster ages provide an accurate relative age scale between regions (e.g., Naylor et al. 2009), age spreads within clusters may be real or could result from observational uncertainties (e.g., Hartmann et al. 1998; Hillenbrand et al. 2008; Preibisch 2012).

The uncertainties in stellar parameters affect our interpretation of stellar evolution. For example, Gullbring et al. (1998) found accretion rates an order of magnitude lower than those of Hartigan et al. (1995) and attributed much of this difference to lower values of extinction. The Gullbring et al. (1998) accretion rates of $10^{-8} M_{\odot} \mathrm{yr}^{-1}$ means that steady accretion in the CTTS phase accounts for a negligible amount of the final mass of a star. However, subsequent near-IR analyses have revised extinctions upward (e.g., White \& Ghez 2001; Fischer et al. 2011; Furlan et al. 2011). These higher extinctions would yield accretion rates of $10^{-7} M_{\odot} \mathrm{yr}^{-1}$, fast enough that steady accretion over the $\sim 2-3$ Myr CTTS phase would account for $\sim 20 \%-50 \%$ of the final stellar mass, or more with the older ages measured by Bell et al. (2013). The uncertainties in stellar properties introduce skepticism in our ability to use young stellar populations to test theories of star formation and pre-main-sequence evolution.

For CTTSs, minimizing the uncertainties in spectral type, extinction, and accretion (often referred to as veiling of the 
Table 1

Observation Setup and Log

\begin{tabular}{|c|c|c|c|c|c|c|c|c|c|}
\hline \multirow[t]{2}{*}{ Telescope } & \multirow[t]{2}{*}{ Dates } & \multirow[t]{2}{*}{ Instrument } & \multirow[t]{2}{*}{ Slit } & \multicolumn{3}{|c|}{ Blue Setup } & \multicolumn{3}{|c|}{ Red Setup } \\
\hline & & & & Grating & Wavelength & Res. & Grating & Wavelength & Res. \\
\hline Palomar & 2008 Jan $18-21$ & DoubleSpec & $1-4^{\prime \prime}$ & B600 & $3000-5700$ & 700 & R316 & $6200-8700$ & 500 \\
\hline Palomar & 2008 Dec $28-30$ & DoubleSpec & $4^{\prime \prime}$ & B600 & $3000-5700$ & 700 & R316 & $6200-8700$ & 500 \\
\hline Keck I & 2006 Nov 23 & LRIS & $0.7-1^{\prime \prime}$ & B400 & $3000-5700$ & 900 & R400 & 5700-9400 & 1000 \\
\hline Keck I & 2008 May 28 & LRIS & $1^{\prime \prime}$ & B400 & $3000-5700$ & 900 & R400 & 5700-9400 & 1000 \\
\hline
\end{tabular}

photosphere by accretion) requires fitting all three parameters simultaneously (e.g., Bertout et al. 1988; Basri \& Bertout 1989; Hartigan \& Kenyon 2003). In recent years, such fits have received increasing attention and have been applied to Hubble Space Telescope (HST) photometry of the Orion Nebula Cluster (da Rio et al. 2010; Manara et al. 2012), broadband optical/ near-IR spectra of two Orion Nebular Cluster stars (Manara et al. 2013a), and to near-IR spectroscopy (Fischer et al. 2011; McClure et al. 2013).

In this project, we analyze low-resolution optical blue-red spectra to determine the stellar and accretion properties of 281 of the nearest young stars in Taurus, Lupus, Ophiucus, the TW Hya Association (TWA), and the MBM 12 Association. This first paper focuses on spectral types and extinctions of our sample. The primary advances are the inclusion of blue spectra to complement commonly used red optical spectra and use of accretion estimates to calculate the effective temperatures and luminosities with a single, consistent approach for a large sample of stars. Discrepancies are found between our results and near-IR based extinction measurements. We then discuss how these uncertainties affect the reliability of age measurements. This work was initially motivated to calculate accretion rates from the excess Balmer continuum emission, which will be described in a second paper. A third paper in this series will discuss spectrophotometric variability within our sample.

\section{OBSERVATIONS}

We obtained low resolution optical spectra with the Double Spectrograph (DBSP; Oke \& Gunn 1982) on the Hale 200 inch telescope at Palomar Observatory on 2008 January 18-21 and 2008 December 28-30, and with the Low Resolution Imaging Spectrograph (LRIS; Oke et al. 1995; McCarthy et al. 1998) on Keck I on 2006 November 23 and 2008 May 28. The entire sample of the 2006 Keck observations was published in Herczeg $\&$ Hillenbrand (2008). The latest spectral types of the 2008 May run were published in Herczeg et al. (2009). The Atmospheric Dispersion Corrector (Phillips et al. 2006) was used for the 2008 May run but was not yet available in 2006 November. Both DBSP and LRIS use a dichroic to split the light into red and blue beams at $\sim 5600 \AA$. Details of the gratings and spectral coverage are listed in Table 1.

On DBSP, the blue light was recorded by the CCD 23 detector, with $15 \mu \mathrm{m}(0$ ('389) pixels in a $2048 \times 4096$ format. The red light was recorded by the Tektronix detector, with $24 \mu \mathrm{m}\left(00^{\prime \prime} 468\right)$ pixels in a $1024 \times 1024$ format. The red detector has since been replaced. On LRIS, the blue E2V and the red LBNL detectors both have $2048 \times 4096$ pixels with a plate scale of 0! 135 .

Our typical observing strategy consisted of 3-10 short (1-60 s) red exposures and 1-2 long (60-900 s) blue exposures obtained simultaneously. Most DBSP observations in 2008 January were obtained with the $2^{\prime \prime}$ width slit, though a few sources were observed with the $1^{\prime \prime}$ or $4^{\prime \prime}$ width slits, adjusted for seeing. All 2008 December observations were obtained with the $4^{\prime \prime}$ width slit. Our LRIS observations were obtained with the 0.7 and 1.0 slits. Seeing during both Palomar runs typically varied from $2^{\prime \prime}-4^{\prime \prime}$, though for a few hours the seeing reached $\sim 1^{\prime \prime}$. The seeing was $\sim 00^{\prime} 8$ and $\sim 0$ '7 during our 2006 November and 2008 May Keck runs, respectively. Seeing was often worse than these measurements for objects at high airmass. The position angle of the slit was set to the parallactic angle for all observations of single stars to minimize slit loss. For binaries, the position angle may be misaligned with the parallactic angle. These observations were timed to occur at low airmass or when the parallactic angle matched the binary position angle.

The images were overscan-subtracted and flat-fielded. Most DBSP spectra were extracted using a 21 pixel $\left(10^{\prime \prime}\right.$ window centered on the source, followed by subtracting the sky as measured nearby on the detector. Binaries with separations $<5^{\prime \prime}$ were extracted simultaneously by assuming a wavelength-dependent point-spread function determined from an observation of a single star observed close in time. The counts from one source are subtracted from the image, yielding a clean extraction of counts from the other source. In several cases, the counts are extracted on only half of the line spread function to further minimize contamination from the nearby component. Each spectrum is then corrected for light loss outside the slit and outside our extraction window based on the measured seeing as a function of wavelength and under the assumption that the point spread function is Gaussian. The light loss is typically $3 \%-10 \%$ and increases to short wavelengths.

\subsection{Flux Calibration}

To calibrate fluxes, spectrophotometric standards (G191B2B, LLT 3864, Hz 44, Feige 110, and Feige 34; see Oke 1990) were observed $\sim 8-13$ times on most nights. On 2008 January 21, G191B2B was observed twice and the night ended early because of snow. The 2006 Keck run included only two spectrophotometric standards and has a large uncertainty in the flux calibration. These spectra were also used to correct telluric features in the red, particularly $\mathrm{H}_{2} \mathrm{O}$ bands at 7200 and $8200 \AA$ A. Windows between $7580-7680$ and $6860-6890 \AA$ are severely contaminated by deep telluric absorption and not used. A different atmospheric transmission curve was calculated for every night and was applied to each spectrum. The correction at $3500 \AA$ ranged from $0.5-0.65 \mathrm{mag} /$ airmass at Palomar and $0.4 \mathrm{mag} /$ airmass at Keck.

The standard deviation in count rates and flux ratios for our 47 DBSP spectra of G191B2B and 9 DBSP spectra of LTT 3864 are listed in Table 2. The flux calibration is based on multiple G191B2B spectra each night, so the standard deviation in flux is not completely independent. The LTT 3864 spectra were observed at airmass $\sim$ three and are all independent data points. The flux calibration within the red channel is $<2 \%$. The absolute flux uncertainty, the cross-calibration between the red and blue spectra, and the relative flux calibration within the blue channel are accurate to $\sim 5 \%$. The quality of the calibration degrades 
Table 2

Flux Calibration

\begin{tabular}{lcc}
\hline \hline Wavelength & G191B2B & LTT 3864 \\
\hline$(\AA)$ & Absolute Scatter in Fluxes \\
\hline 3500 & 0.067 & 0.087 \\
4300 & 0.056 & 0.046 \\
5400 & 0.041 & 0.047 \\
6300 & 0.063 & 0.091 \\
8400 & 0.061 & 0.089 \\
\hline Flux Ratio & Scatter in Flux Ratios \\
\hline$F_{7020} / F_{7140}$ & 0.007 & 0.005 \\
$F_{8400} / F_{6300}$ & 0.016 & 0.014 \\
$F_{6300} / F_{5400}$ & 0.057 & 0.101 \\
$F_{4300} / F_{5400}$ & 0.034 & 0.051 \\
$F_{3500} / F_{5400}$ & 0.048 & 0.087 \\
\hline
\end{tabular}

to $\sim 10 \%$ at a high airmass. When extracting close binaries the absolute accuracy in flux is $\sim 30 \%$, particularly for secondaries that are much fainter than the primary or for observations where the seeing was larger than the binary separation.

Fringing is often apparent in DBSP spectra at $<3700 \AA$ for observations obtained at a high airmass and is likely a result of telescope vignetting. However, accurate continuum fluxes in this region are still measurable in large wavelength bins.

\subsection{Sample Selection}

At Palomar, we tried to observe all visually bright targets in Taurus with spectral type (SpT) between K0-M4 and targets that were known as of 2008 (see review by Kenyon et al. 2008). A few Taurus objects with spectral types earlier than M4 were missed due to clerical errors. Many new Taurus members were identified after 2008 and are not included here. For later spectral types, our sample is far from complete and is biased to the targets that were optically brightest because they had the best chance of having $U$-band detections. We also obtained a complete sample of the known objects in MBM 12 (Luhman 2001) and some of the TWA. In some cases, the membership of the star in the parent cloud is uncertain. The stars from the HBC with numbers between 352-357 that were observed here are consistent with low gravity but are likely not members of Taurus (Kraus \& Hillenbrand 2009).

During our Keck runs, we observed many brown dwarfs to measure accretion rates at the lowest mass end of the initial mass function. Our 2006 Keck run was focused on Taurus, while the 2008 run included objects in the Ophiucus, Lupus, and Corona Australus molecular clouds and the Upper Sco OB Association.

The source list and final properties for the young stars in our sample are listed in Appendix C (see Table 14). Multiple spectra were obtained for 59 targets, including $>3$ observations of 29 bright and famous targets. We also obtained spectra of 40 main sequence $\mathrm{K}$ and $\mathrm{M}$ dwarfs with known spectral type (Kirkpatrick et al. 1993). The spectra from these stars are used when describing field star spectra in Section 3 but are otherwise not discussed.

Our sample includes some brown dwarfs. For simplicity, all objects are referred to as stars regardless of their estimated mass.

\section{ESTABLISHING SPECTRAL TEMPLATES FOR T TAURI STARS}

The necessary ingredients for age and accretion calculations are the stellar mass, radius, and accretion luminosity. These

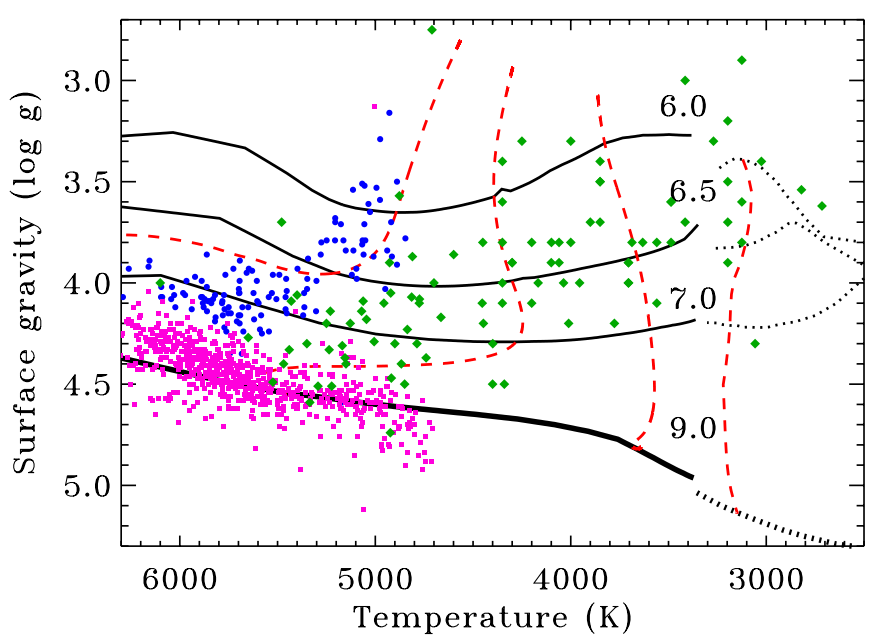

Figure 1. Isochrones of gravity vs. temperature from Tognelli et al. (2011) for $M_{*}>0.3 M_{\odot}$ (solid horizontal lines) and Baraffe et al. (2003) for $M_{*}<0.3 M_{\odot}$ (dotted horizontal lines), plotted analogous to an H-R diagram. Pre-mainsequence tracks of 2.0, 1.0, 0.5, and $0.2 M_{\odot}$ stars are shown from left to right (red dashed lines). The gravity increases as the star contracts during the pre-mainsequence evolution (log age as labeled). The gravities of pre-main-sequence stars (green diamonds from Stassun et al. 2007; Santos et al. 2008; Taguchi et al. 2009; D'Orazi et al. 2009, 2011; Biazzo et al. 2011, 2012) are similar to the gravities of luminosity class IV subgiants (blue circles, $\log g=3.5-4.5$, Valenti \& Fischer 2005) and less than the main sequence (thick horizontal line at the bottom, main sequence data points as purple squares from Valenti \& Fischer 2005).

(A color version of this figure is available in the online journal.)

parameters require measurements of the stellar effective temperature, the photospheric flux, and the extinction. While analysis of optical spectra of main sequence stars from photospheric features is usually straightforward, the lower gravity and presence of accretion complicates the measurement of stellar properties of young stars. Pre-main-sequence stars have similar surface gravity to cool subgiants of luminosity class IV and are offset from the luminosity class V field dwarfs (Figure 1; see also Gray 2005). However, gravity measurements for pre-main-sequence stars are challenging because unlike subgiants, they are fast rotators.

A typical weak lined TTS spectrum is covered with photospheric absorption in molecular bands and atomic lines, along with chromospheric emission in $\mathrm{H}$ Balmer and $\mathrm{Ca}$ II $\mathrm{H} \& \mathrm{~K}$ lines. Accretors usually show strong emission in those lines, along with weak emission in the Ca II infrared triplet, in He I lines, in an accretion continuum, and often in forbidden lines. Some accretors show many additional lines, mostly of Fe (e.g., Hamamn \& Persson 1992; Beristain et al. 1998). The accretion continuum reduces the depth of photospheric absorption lines, a process that is called "veiling." The veiling is defined as $r_{\lambda}=F_{\text {veil }} / F_{\text {phot }}$ at a given wavelength $\lambda$. The veiling at $5700 \AA$, $r_{5700}$, is typically between $0.1-1$, though in rare cases the veil may cover the photospheric emission (Hartigan et al. 1995; Fischer et al. 2011). The flux in the photospheric and emission lines are often reduced by extinction.

In this section, we describe our initial approach for measuring the properties of the stars in our sample, with an emphasis on quantifying the approach for measuring $\mathrm{SpT}, A_{V}$, and the accretion continuum flux. The analysis in this section results in a grid of extinction-corrected spectral templates and an approach for including the accretion in spectral type and extinction measurements, which are then applied to the full data set in Section 4. 
Table 3

Spectral Indices

\begin{tabular}{|c|c|c|c|c|c|c|c|c|}
\hline Name & $\begin{array}{c}\text { Continuum Range } \\
\text { (C) }\end{array}$ & $\begin{array}{l}\text { Band Range } \\
\text { (B) }\end{array}$ & Feature & $x$ & SpT & Range & Zero-pt & $\mathrm{rms}^{\mathrm{a}}$ \\
\hline$G$-band & $4550-4650$ & $4150-4250$ & $G$-band & $\mathrm{C} / \mathrm{B}$ & $-25.6+29.96 x$ & G & G0 & $\sim 1$ \\
\hline R5150 & $4600-4700$ & $5050-5150$ & $\mathrm{MgH}$ & $\frac{F(5100)}{F(4650)} \frac{F_{\text {line }}(4650)}{F_{\text {line }}(5100)} \mathrm{b}$ & $-29.7+28.3 x$ & K0-M0 & K0 & 1.0 \\
\hline $\mathrm{TiO} 6250$ & $6430-6465$ & $6240-6270$ & $\mathrm{TiO}$ & $\log \left(\frac{C}{B}-1\right)$ & $3.20-5.43 x+1.73 x^{2}$ & (M0-M4) & M0 & $\ldots$ \\
\hline TiO 6800 & $6600-6660,6990-7050$ & $6750-6900$ & $\mathrm{TiO}$ & $\mathrm{C} / \mathrm{B}$ & $-15.37+19.77 x$ & K5-M0.5 & K0 & $\ldots$ \\
\hline TiO 7140 & 7005-7035 & $7130-7155$ & $\mathrm{TiO}$ & $\log \left(\frac{C}{B}-1\right)$ & $4.36+6.33 x+1.57 x^{2}$ & M0-M4.5 & M0 & $0.42^{\mathrm{c}}$ \\
\hline TiO 7700 & $8120-8160^{\mathrm{d}}$ & $7750-7800$ & $\mathrm{TiO}$ & $\mathrm{C} / \mathrm{B}$ & $0.11+2.27 x$ & M3-M8 & M0 & 0.21 \\
\hline TiO 8465 & $8345-8385$ & $8455-8475$ & $\mathrm{TiO}$ & $\mathrm{C} / \mathrm{B}$ & $-0.74+4.21 x$ & M4-M8 & M0 & 0.18 \\
\hline
\end{tabular}

Notes. All spectral indices are calculated from the median flux in the spectral range.

${ }^{\text {a }}$ SpT rms calculated from the literature SpT (R5150, TiO 6800), Kirkpatrick SpT (TiO 7140), and Luhman SpT (TiO 7700, 8465).

b The observed flux ratio is divided by the same ratio obtained from a linear fit to the 4650-5300 A region, see the text.

${ }^{c} 0.3$ between $\mathrm{M} 1$ and M4, 0.8 earlier than M1.

d The 8120-8160 ̊̊ continuum range should be used only for spectra that are corrected for telluric $\mathrm{H}_{2} \mathrm{O}$ absorption.

\subsection{Quantification of Spectral Indices}

In this subsection, atlases of low-resolution optical spectra are used to establish a set of quantified spectral indices for young stars. The following descriptions are divided by spectral type, each of which is sensitive to a different spectral index. Spectral typing of young stars has typically relied on eyeball comparisons to a sequence of spectral standards. While that approach can be very accurate, a quantified approach allows for greater consistency between different sets of eyes. A quantified approach also readily accounts for accretion and extinction by calculating over a grid of values to find a best fit solution.

The full set of spectral indices discussed in this paper is listed in Table 3. By design, our focus is on $\mathrm{K}$ and $\mathrm{M}$ stars. The M-dwarf spectral types rely on the depth of TiO and VO absorption bands (hereafter referred to as $\mathrm{TiO}$ ), which start to become detectable at $\sim \mathrm{K} 5$. For $\mathrm{K}$ dwarfs, a spectral type index is developed based on the $5200 \AA$ absorption feature, which is a combination of $\mathrm{MgH}, \mathrm{Mg} \mathrm{b}$, and Fe I (e.g., Rich 1988). The spectral typing of BAFG stars relies on a visual comparison of the $G$ band and absorption in $\mathrm{H}$ and $\mathrm{Ca}$ lines.

The quantification of spectral typing provides an objective and repeatable method to measure spectral types with precision. The quantified prescriptions of $\mathrm{M}$ dwarfs are similar to those of Slesnick et al. (2006) and Riddick et al. (2007), while the prescriptions for earlier spectral types are similar to those developed by, e.g., Worthey et al. (1994) and Covey et al. (2007). The spectral indices described here are calculated from the median flux in the relevant spectral range and are tailored to low spectral resolution. These spectral indices are then combined with an accurate flux calibration and blue spectra to measure accretion and extinction simultaneously (see Section 4). In several cases, the spectral index is changed to a log scale to provide a better fit between spectral type and spectral index. The TiO-7700 spectral index defined here uses a continuum region that overlaps with telluric $\mathrm{H}_{2} \mathrm{O}$ absorption and should only be used when telluric calibrators are obtained contemporaneously. Use of indices can also be problematic if the spectrum is either not flux calibrated or not corrected for extinction. Converting spectral indices to accurate spectral types requires high signalto-noise ratio $(\mathrm{S} / \mathrm{N})$ in the $\sim 30 \AA$ integration bins and an accurate relative flux calibration (for example, see Table 2 for our flux calibration relevant to the TiO-7140 index). A $2 \%$ error in the $\mathrm{TiO}$ indices typically leads to an error of $0.1-0.2$ subclasses in spectral type.
Scatter in these quantified relationships are caused by metallicity and gravity differences between stars. The metallicity of nearby young associations is uniform (e.g., Padgett 1996; Santos et al. 2008; D'Orazi et al. 2011). Gravity differences between 1-10 Myr may be significant and are discussed but are not fully investigated.

In the following subsections, we describe how these spectral indices are used to measure spectral types. Each spectral index is sensitive to different spectral types and is discussed separately, beginning with the coolest stars in our sample.

\subsubsection{Spectral Types of M Stars}

The majority of stars in our sample are M stars. At optical wavelengths, $M$ stars are easily identified from the presence of strong TiO absorption bands. Kirkpatrick et al. (1991, 1993), hereafter Kirkpatrick, established a grid of M-dwarf spectral type standards from field stars. Reid et al. (1995), hereafter PMSU, quantified relationships between spectral type and the depth of TiO bands at $7100 \AA$ from moderate resolution optical spectra based on the Kirkpatrick et al. (1991) sequence.

Luhman (1999); Luhman et al. (2003), hereafter Luhman (also includes, e.g., Luhman 2004, 2006), recognized that for pre-main-sequence stars, the depth of $\mathrm{TiO}$ features deviates from dwarf stars because of lower gravity (see also Gullbring et al. 1998). Luhman developed a spectral type sequence for young M dwarfs later than M5 based on a hybrid of field dwarf and giant stars, since TTSs are typically luminosity class IV. For stars earlier than M5, Luhman relied on the Kirkpatrick grid along with the Allen \& Strom (1995) red spectroscopic survey of standards. Although the Luhman spectral sequence is well accepted and widely used, it has no standards or quantified conversions between spectral index and spectral type. As a consequence, spectral types based on the Luhman method are likely less precise when applied by authors other than Luhman himself.

Our quantified spectral type sequence is derived from the methods established in those seminal works. In the following analysis, the objects in the PMSU catalog are all assigned a spectral type based on their TiO5-SpT conversion, which is accurate to $\sim 0.5$ subclasses between $\mathrm{K} 7-\mathrm{M} 6$. The TiO5 spectral index, the flux ratio of 7130-7135 to 7115-7120 , requires flux measurements in narrow regions and is not possible to calculate from our low resolution spectra. The Luhman sequence discussed here is from a set of 54 young stars spanning M0.5-M9.5 provided by K. L. Luhman (2008, private communication). 


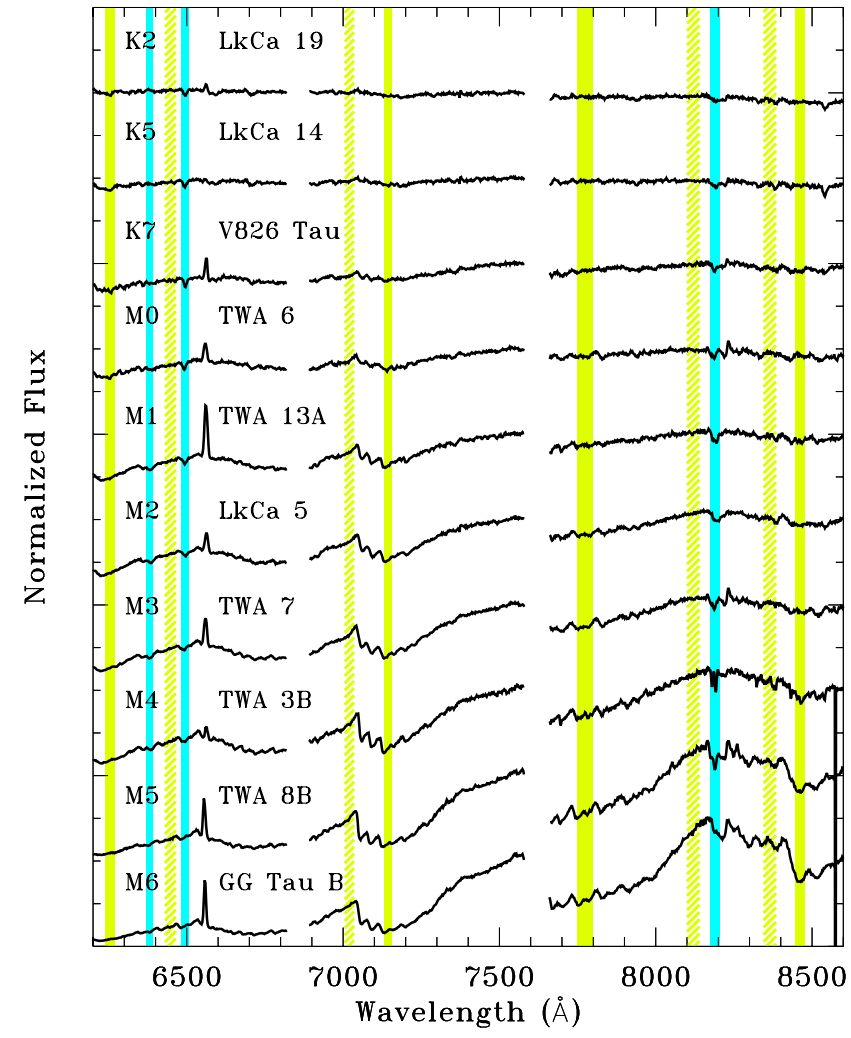

Figure 2. Red spectral sequence from K3-M5.5. Regions used for TiO band indices are highlighted in yellow. Selected gravity-sensitive absorption in $\mathrm{CaH} \lambda 6382, \mathrm{Fe}$ I $\lambda 6497$, and the $\mathrm{Na}$ I $\lambda 8189$ doublet are highlighted in blue.

(A color version of this figure is available in the online journal.)
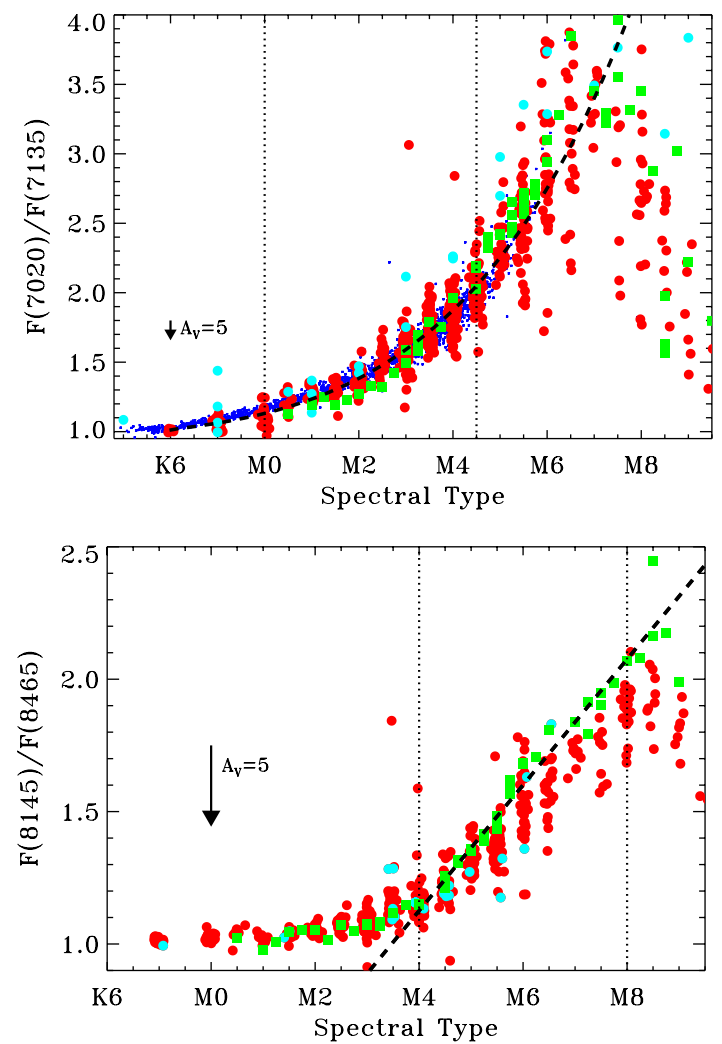

Four prominent $\mathrm{TiO}$ bands are present in our red spectra (see Figure 2 and Table 3). Figure 3 compares the spectral types and four spectral indices for the PMSU, Kirkpatrick, and Luhman samples. For stars earlier than M5, the Luhman relationship between SpT and TiO depth for young stars was intended to follow the Kirkpatrick et al. (1991) results. However, for objects from M0 to M3, the Luhman spectral types are $\sim 0.5$ subclasses later than the median Kirkpatrick spectral type (TiO7140 and TiO-6200 spectral indices). For the spectral types later than M5, gravity differences between field M dwarfs and pre-main-sequence $M$ dwarfs lead to the Luhman spectral types being slightly earlier than the median Kirkpatrick object (as discussed by Luhman). For M dwarfs earlier than M4, we adopt the spectral type sequence of Kirkpatrick, which may introduce a small offset between our spectral types and Luhman spectral types. For M dwarfs later than M4, we adopt the spectral type sequence of Luhman. Several additional $\mathrm{TiO} / \mathrm{VO}$ bands are detected at blue wavelengths and are not well studied (see Figure 4). While our initial approach does not consider these bands, the final spectral types are calculated from a best fit to a spectral sequence using the full optical spectrum. ${ }^{5}$

M4-M8. Objects later than M4 have spectral types assessed from the TiO 7700 and $8500 \AA$ bands, with a conversion from spectral index to spectral type calculated from the sequence of objects provided by Luhman. An uncertainty of 0.2 subclasses is assigned based on the change in feature strength versus subclass and on the standard deviation in the fits to the Luhman objects. This uncertainty is consistent with that assigned by Luhman.

\footnotetext{
5 The TiO 7140 index was developed by Slesnick et al. (2006). Our definition uses a slightly different continuum region.
}
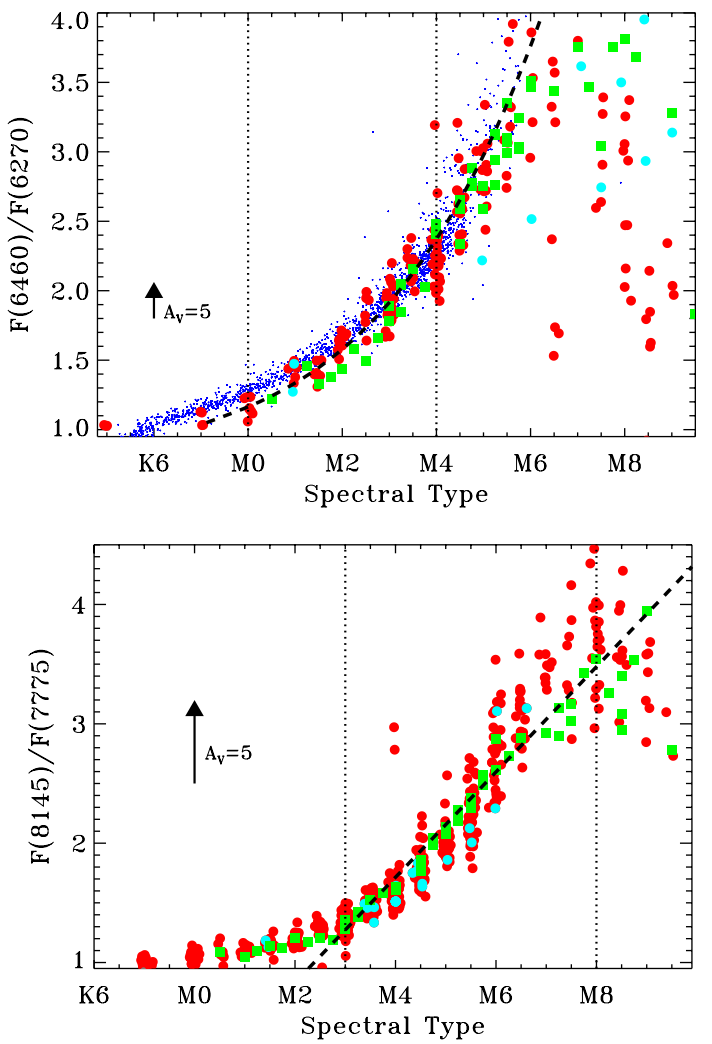

Figure 3. Relationships between spectral index and spectral type for four TiO bands. Large circles are calculated from Kirkpatrick (red are main sequence and cyan are giant stars), small blue dots are from PMSU, and green points are from spectra provided by Luhman. Best-fit conversions between spectral index and spectral type are shown as the dashed line and quantified in Table 3 . The vertical dotted lines show the spectral type range where these relationships are used. The arrows show how the listed extinction $A_{V}=5$ mag. would shift the index.

(A color version of this figure is available in the online journal.) 

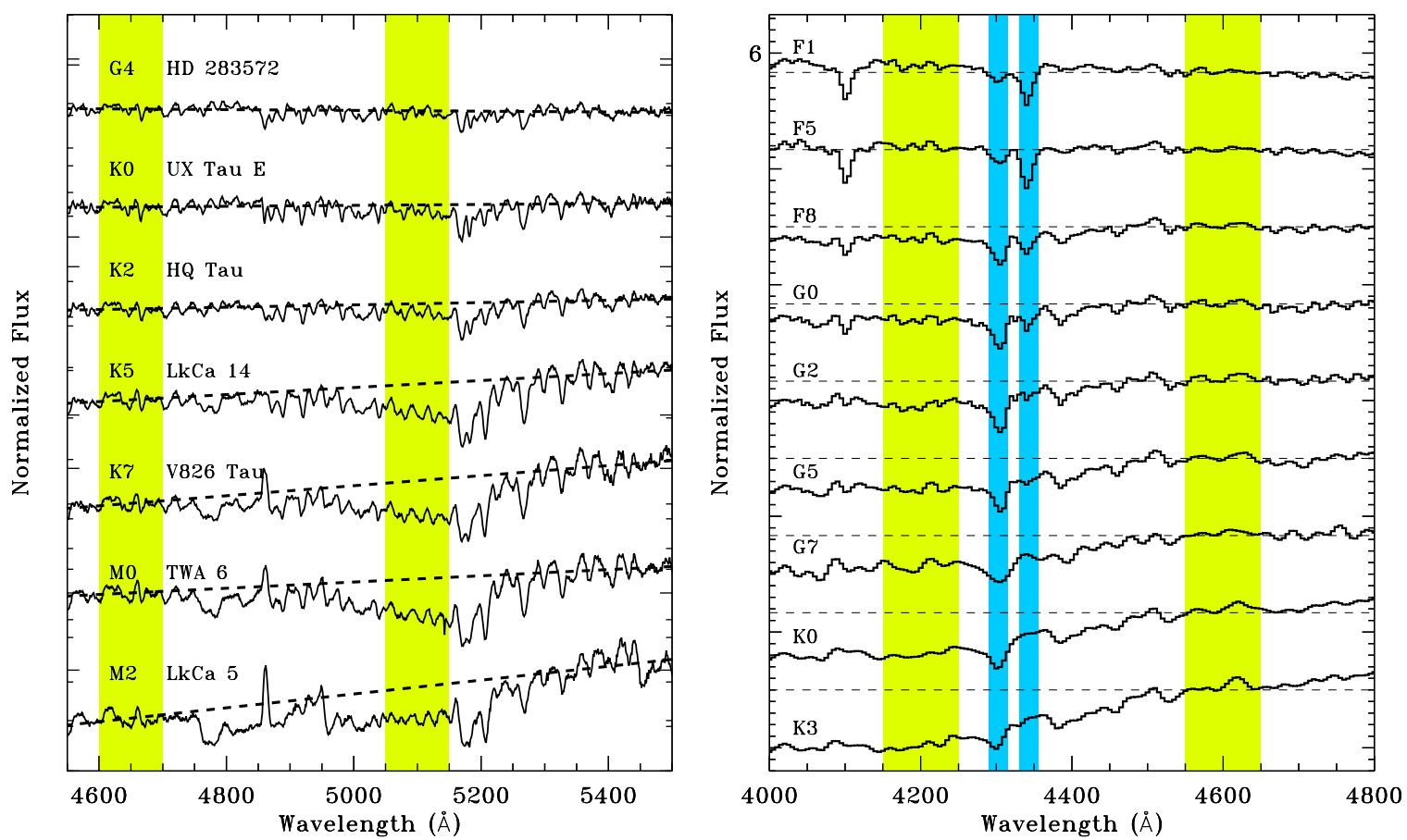

Figure 4. Blue spectral sequence used to measure SpT for K stars (left, from this work) and FG stars (right, luminosity class IV stars from Pickles). K-dwarf spectra show a dip in flux just shortward of the $\mathrm{Mg}$ I $\lambda 5150$ line, which gets stronger to later $\mathrm{K}$ and to higher gravity. The right panel shows how the $\mathrm{H} \gamma$ and the CaI $\lambda 4227$ lines (shaded blue regions) vary with SpT from F1 to K3. The shaded yellow regions show the ranges used to calculate spectral indices for G and K dwarfs.

(A color version of this figure is available in the online journal.)

MO-M4. The TiO band at $7140 \AA$ is most reliable for early-to-mid M-dwarfs. Within the Kirkpatrick sample between M0-M4.5, the standard deviation of the index-determined SpT and adopted $\mathrm{SpT}$ is 0.4 subclasses. The relative accuracy of spectral typing within a single star-forming region is likely better than 0.40 subclasses because the Kirkpatrick lists SpT at only 0.5 subclass intervals and because the metallicity should be uniform in samples of nearby star forming regions but not in field dwarfs. The TiO $6200 \AA$ band is also sensitive to early-tomid M dwarfs, with a standard deviation of 0.22 subclasses for spectral types M0-M4 within the Kirkpatrick sample. However, few Kirkpatrick objects were observed at $6200 \AA$, and the PMSU sample is systematically offset from the Kirkpatrick sample in this $\mathrm{TiO}$ feature. As a consequence, we do not use this relationship here to derive spectral types.

\subsubsection{KO-MO.5 Spectral Types}

Figure 4 (left panel) shows that K dwarfs are characterized by $\mathrm{MgH}$ and $\mathrm{Mg}$ b absorption at $\sim 5150 \AA$. This dip is not present in G-type stars. We define a spectral index, $R(5150)$,

$$
R(5150)=\frac{F(5100)}{F(4650)} \times \frac{F_{\text {line }}(4650)}{F_{\text {line }}(5100)},
$$

where $F(\lambda)$ is the flux in a $100 \AA$ wide band around $\lambda$, and $F_{\text {line }}(4650) / F_{\text {line }}(5100)$ is the flux ratio expected at those same wavelengths based on the spectral slope obtained in a linear fit to the $\lambda=4650$ and $\lambda=5450 \AA$ spectral regions. Dividing by the $\mathrm{F}(5100) / \mathrm{F}(4650)$ ratio calculated from the linear fit accounts for extinction.

Figure 5 shows the relationship between $R(5150)$ versus literature spectral type for stars with little or no accretion. The spectral types earlier than M0 are obtained from the literature, usually from high-resolution spectra (Basri \& Batalha

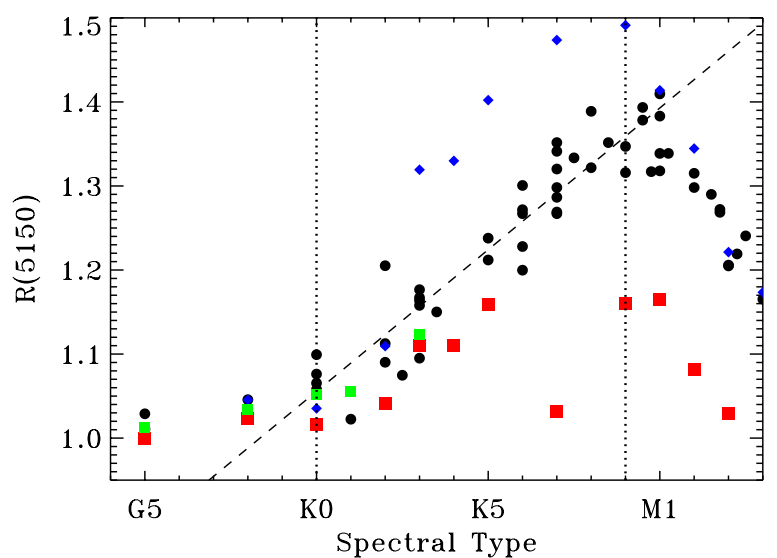

Figure 5. Spectral type vs. the spectral index $R 5150$ for young K-dwarfs (see Figure 4) for young stars in our sample (black circles), Pickles templates of luminosity class IV (green squares), V (blue diamonds), and the average of Pickles templates of luminosity class III and V (red squares). The vertical dotted lines show the spectral type range where these relationships are used. The SpT used in this analysis are all from the literature and may differ from the SpT calculated in this work. The turnover around M1 occurs when TiO absorption becomes prominent enough to affect the flux ratio.

(A color version of this figure is available in the online journal.)

1990; White \& Hillenbrand 2004; White et al. 2007), and are supplemented by some low resolution spectral types from Luhman. Spectral types later than M0 are calculated from the TiO spectral indices. Figure 5 also shows $R(5150)$ versus SpT from the compilation of low resolution spectral atlases by Pickles (1998). The $R(5150)$ index is similar to that of luminosity class IV subgiants and to the average index obtained by adding spectra of dwarfs and giants (luminosity class III + luminosity class V). The relationship is gravity-sensitive and should be applied only to pre-main-sequence K stars. 

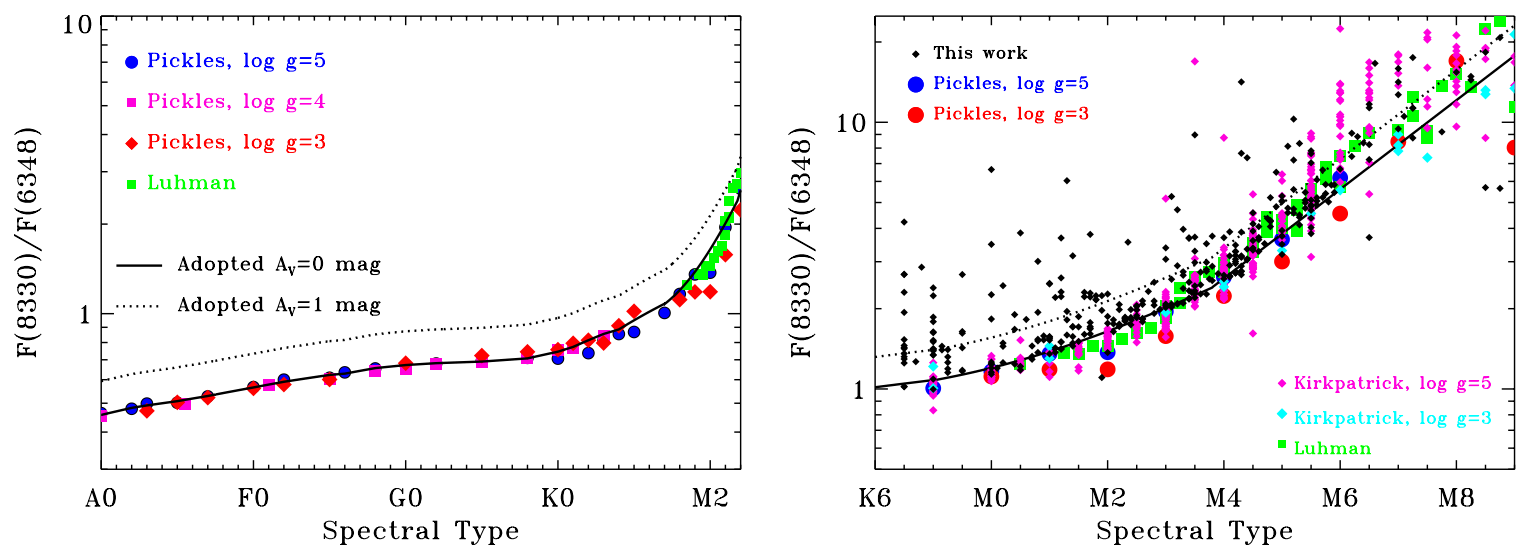

Figure 6. Flux ratio $F_{\text {red }}=F(8330) / F(6448)$ vs. SpT for A-M stars (left) and focused in on K-M stars (right), for SpT calculated from the spectral indices in described in Section 3. Extinction can then be measured by comparing $F_{\text {red }}$ to the expected ratio for a given spectral type.

(A color version of this figure is available in the online journal.)

The standard deviation between calculated and literature spectral types between K0 and M0 is 1.0 subclasses. Some of this scatter is attributable to uncertainty in literature spectral type, which typically claim an accuracy of one to two subclasses, and to studies listing integer steps in subclass. We assign an uncertainty of one subclass between $\mathrm{K} 0-\mathrm{M} 0$ for this relationship.

The $\mathrm{TiO} 6800 \AA$ absorption band is detectable for spectral types K5 and later. From K5-M0, the Kirkpatrick objects are about one subclass later than the Pickles libraries. The PMSU data are also shown, though the PMSU TiO-5 index is not reliable at spectral types earlier than $\mathrm{K} 7$. We include a spectral type K8 as an intermediate between K7 and M0. Spectral types between K6-M0.5 are assigned an uncertainty of $\sim 0.5$ subclasses. Most accreting stars in this spectral type range have a spectral type uncertainty of one subclass. Within this range there may be an additional systematic uncertainty of $\sim 0.5$ subclasses between our spectral types and those of Luhman.

\subsubsection{B, A, F, and G Spectral Types}

By design, only a few objects in our sample have a spectral type earlier than K. Spectral types for these objects are measured by visual comparison to Pickles templates. The shape of the $G$ band helps to determine $\mathrm{G}$ spectral types, while the absence of the $G$ band requires that the star be F or later (e.g., Fraunhofer 1814; Cannon 1912; Covey et al. 2007). Both G and F spectral types are also measured from the relative strengths of the $4300 \AA$ line and the nearby $\mathrm{H} \gamma$ line. Hotter stars have spectral types measured from the strength of Balmer lines and the $\mathrm{Ca}$ II $\mathrm{H}$ $\& \mathrm{~K}$ lines. The strength of the $\mathrm{Ca}$ II $\mathrm{K}$ line is particularly important for discriminating between $\mathrm{B}$ and early A spectral types (e.g., Mooley et al. 2013), although the absorption may be filled in with emission. More rigorous approaches to spectral typing large samples of BAF stars are described by Hernandez et al. (2004) and Alecian et al. (2013). Some uncertainty in our classification is introduced by emission and possible wind absorption in $\mathrm{H}$ and $\mathrm{Ca}$ lines.

\subsection{Photospheric Extinction Measurements}

Extinction measurements require a comparison of observed flux ratios or spectral slopes to the same flux ratios or slopes from a star with the same underlying spectrum and a known extinction. For non-accreting stars, this flux ratio can be compared to a photospheric template with similar gravity and negligible extinction. The effect of accretion on photospheric extinction measurements is discussed in Section 3.4.

The extinction curve used in this paper is from (Cardelli et al. 1989) with the average interstellar value for total-toselective extinction, $R_{V}=3.1$. The value for $R_{V}$ increases to 5.5 for larger dust grains found deep in molecular clouds when $A_{V} \sim 20$, far larger than any extinction measured in this optical sample (Indebetouw et al. 2005; Chapman et al. 2009). To keep the amount of analysis reasonable and for consistency, $R_{V}$ is assumed to be constant throughout our sample when possible. A few stars could only be fit with higher $R_{V}$ (see Appendix C).

Initial extinctions calculated in this paper and applied to a spectral template grid are based on the flux ratio $F_{\text {red }}=$ $(F(8330) / F(6448))$ (flux at $8330 \AA$ to that at $6448 \AA$ ), although our final extinctions use the full blue-red spectra (see Section 4 ). The ratio $F_{\text {red }}$ is affected by the photospheric temperature, accretion spectrum, and extinction. These wavelengths are selected to avoid telluric and $\mathrm{TiO}$ absorption bands and to maximize the wavelength difference of the two bands while requiring both to be in the red detector.

Figure 6 shows $F_{\text {red }}$ versus spectral type for the full range of $\mathrm{SpT}$ (left) and for late $\mathrm{K}$ and $\mathrm{M}$ dwarfs (right). The curve of $F_{\text {red }}$ versus $\mathrm{SpT}$ for $A_{V}=0$ for stars earlier than M0 is based on the Pickles spectral atlas, with giants and dwarf having similar values. Objects provided by Luhman are also included to help fill the grid for stars with SpT later than M5. The value of $F_{\text {red }}$ diverges between the young star and the field dwarf sample at SpT later than M4, which confirms the approach of Luhman to calculate a new SpT-effective temperature conversion for young stars. Within this range, a 0.25 uncertainty in SpT subclass leads to a 0.15 mag uncertainty in $A_{V}$.

At spectral types earlier than K5, we lack the necessary coverage in spectral types of unreddened stars to establish a reliable baseline in $F_{\text {red }}$ versus spectral type to calculate extinctions. Instead, we interpolate $F_{\text {red }}$ over the spectral type grid from the flux-calibrated Pickles compilation of stars with luminosity class V. Most objects in the Pickles compilation have fluxes accurate to $\sim 1 \%$. For $\mathrm{K}$ dwarfs, $F_{\text {red }}$ is about $5 \%$ larger for objects of luminosity class III and IV relative to V. We therefore multiply the interpolated curve by $3 \%$, intermediate between luminosity classes III and V and assess a $3 \%$ uncertainty in the flux baseline. This uncertainty introduces a 0.12 mag uncertainty in $A_{V}$ measurements. For $\mathrm{F}$ and $\mathrm{G}$ dwarfs, $F_{\text {red }}$ is not very sensitive to changes in SpT, with an average change of $2 \%$ 
Table 4

Derived Parameters for Grid of Weak-lined T Tauri Stars

\begin{tabular}{|c|c|c|c|}
\hline Star & $\mathrm{SpT}^{\mathrm{a}}$ & $\begin{array}{c}A_{V}^{\mathrm{a}} \\
(\mathrm{mag})\end{array}$ & $\begin{array}{l}T_{\text {eff }} \\
(\mathrm{K})\end{array}$ \\
\hline НBC 407 & K0 & 0.80 & 5110 \\
\hline HBC 372 & $\mathrm{~K} 2$ & 0.63 & 4710 \\
\hline LkCa 14 & K5 & 0.00 & 4220 \\
\hline MBM12 1 & K5.5 & 0.00 & 4190 \\
\hline TWA 9A & K6.5 & 0.00 & 4160 \\
\hline V826 Tau & $\mathrm{K} 7$ & 0.38 & 4020 \\
\hline V830 Tau & K7.5 & 0.40 & 3930 \\
\hline TWA 6 & M0 & 0.00 & 3950 \\
\hline TWA 25 & M0.5 & 0.00 & 3770 \\
\hline TWA 13S & M1.0 & 0.00 & 3690 \\
\hline $\mathrm{LkCa} 4$ & M1.5 & 0.00 & 3670 \\
\hline LkCa 5 & M2.2 & 0.27 & 3520 \\
\hline $\mathrm{LkCa} 3$ & M2.4 & 0.00 & 3510 \\
\hline TWA 8A & M3.0 & 0.00 & 3390 \\
\hline TWA 9B & M3.4 & 0.00 & 3340 \\
\hline J1207-3247 & M3.5 & 0.00 & 3350 \\
\hline TWA 3B & M4.1 & 0.00 & 3120 \\
\hline XEST 16-045 & M4.4 & 0.00 & 3100 \\
\hline J2 157 & M4.7 & 0.41 & 3050 \\
\hline TWA 8B & M5.2 & 0.00 & 2910 \\
\hline MBM12 7 & M5.6 & 0.00 & 2890 \\
\hline V410 X-ray 3 & M6.5 & 0.25 & 2830 \\
\hline Oph 1622-2405A & M7.25 & 0.00 & 2750 \\
\hline $2 \mathrm{M} 1102-3431$ & M8.5 & 0.00 & 2590 \\
\hline
\end{tabular}

Note. ${ }^{\text {a }}$ From red spectrum, may differ from final SpT, $A_{V}$.

per subclass so that a one-subclass $\mathrm{SpT}$ uncertainty leads to a 0.07 mag. uncertainty in $A_{V}$.

\subsection{A Grid of Pre-main-sequence Spectral Types}

Based on the previous descriptions, a grid of photospheric spectral templates are established and listed in Section 4. Templates at spectral types earlier than $\mathrm{K} 0$ are obtained from the Pickles library because of very sparse coverage in our own data. At K0 and later, weak lined TTSs with low extinctions are selected from our spectra for use as photospheric templates. This criterion leads to the selection of many TWA objects for our grid. The conversion from the spectra to temperature and luminosity are described in the following two subsubsections.

This set of stars is then combined into a grid. Two separate spectral sequences are calculated from stars at $\sim$ one subclass intervals. Between K5-M6, the grids comprise of every second star in Table 4 and are therefore independent. The two grids are then linearly interpolated at 0.1 subclasses (earlier than M0) and 0.05 subclasses (later than M0) and are averaged to create a final spectral grid. Therefore, the photospheric template at all classes between K6-M5.5 includes the combination of three to four stars. This method minimizes the problems introduced by any single incorrect spectral type or extinction within this spectral sequence.

Unresolved binarity affects photospheric measurements of both our spectral grid and our target stars. Among the known multiple systems in our grid, V826 Tau is a near-equal mass spectroscopic binary, so the combined optical spectrum would have a very similar spectrum as both components. LkCa 5 has a very low-mass companion (Kraus et al. 2011) that contributes a negligible amount of flux at optical wavelengths. Although LkCa 3 is a quadruple system consisting of two spectroscopic binaries (Torres et al. 2013), the global spectral type and

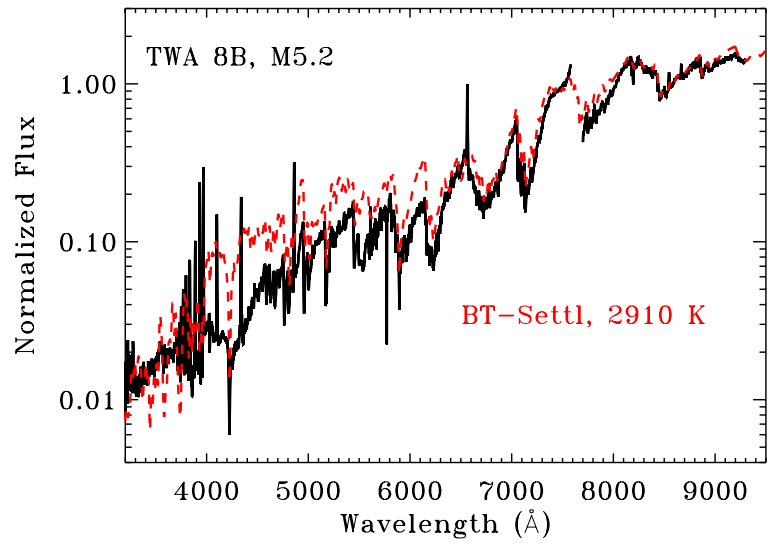

Figure 7. Spectrum of the M5.2 WTTS TWA 8B, compared with BT Settl models of the best-fit temperature. The spectra are scaled to unity at $7325 \AA$. The synthetic spectrum significantly overproduces emission between 4000-6000 A. The TiO bands are deeper in the observed spectrum than in the synthetic spectrum.

(A color version of this figure is available in the online journal.)

extinction is reasonable compared to other stars of similar SpT. In the spectral fits described in Section 4, the combined use of multiple templates for any given star should minimize the problems introduced by known and unknown binarity in the templates.

\subsubsection{Conversion from Spectral Type to Effective Temperature}

The standard conversion from SpT to effective temperature for young stars is based on the work of Schmidt-Kaler (1982) and Straizys (1992), as compiled by Kenyon \& Hartmann (1995). Luhman updated this conversion for M-dwarf TTSs, based on a scale intermediate between giants and dwarfs. Synthetic M-dwarf spectra from model atmospheres have advanced considerably since Luhman et al. (2003) established this conversion. Rajpurohit et al. (2013) recently obtained a new scaling between spectral type and temperature for $\mathrm{M}$ dwarfs by comparing BTSettl synthetic spectra calculated from the Phoenix code (e.g., Allard \& Hauschildt 1995; Allard et al. 2012) to observed lowresolution spectra. A similar approach by Casagrande et al. (2008) with the Cond-Gaia synthetic spectra yielded much lower temperatures than Rajpurohit et al. (2013) for the same spectral type.

An initial comparison between our standard grid and Phoenix/BT-Settl synthetic spectra with CFITSIO opacities and gravity $\log g=4.0$ (Allard et al. 2012; Rajpurohit et al. 2013) reveals good agreement between the observed and synthetic spectra for temperatures higher than $\sim 3200 \mathrm{~K}$. Discrepancies between the observed and modeled depths of TiO absorption bands are problematic at cooler temperatures (Figure 7). We speculate that some of these differences may be explained with uncertainties in the strengths of $\mathrm{TiO}$ transitions and in the strength of continuous optical emission produced by warm dust grains in the stellar atmosphere. Details of these comparisons and fits of the synthetic spectra to observed spectra are described in Appendix B.

An effective temperature scale for pre-main-sequence stars is derived by fitting Phoenix/BT-Settl synthetic spectra to our spectral type grid (K5-M8.5) and Pickles luminosity class IV stars (F-K3). Figure 8 and Table 5 compares our new $\mathrm{K}$ and M-dwarf temperature scale to other pre-main-sequence and 


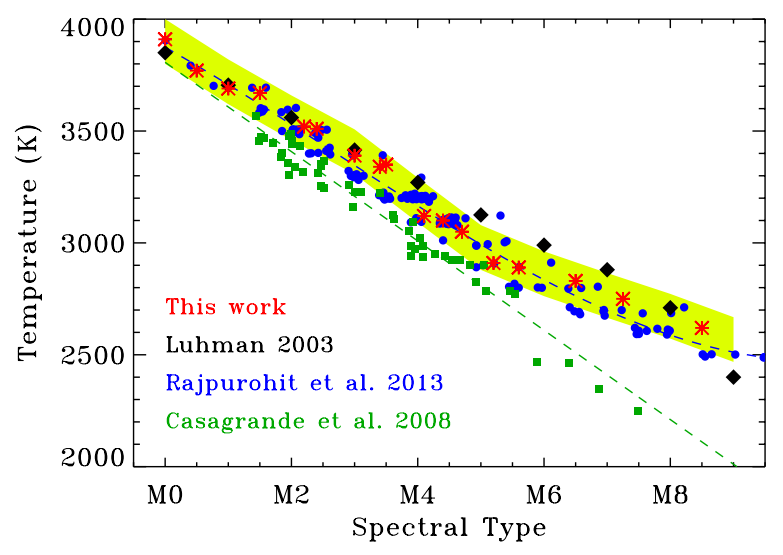

Figure 8. Conversion from spectral type to effective temperature from Luhman et al. 2003 (black diamonds), Rajpurohit et al. 2013 (blue circles, with best-fit polynomial shown as dashed blue line), Casagrande et al. 2008 (green squares, with best-fit line shown as dashed green line), and this work (red asterisks). Small spectral type and temperature changes are randomly applied to the Rajpurohit et al. data so that each point is visually displayed. The shaded yellow region shows the approximate temperature range at constant spectral type derived from atmosphere models, accounting for uncertainties in the comparison between model and observed spectra.

(A color version of this figure is available in the online journal.)

Table 5

Spectral Type to Temperature Conversions

\begin{tabular}{lccccccc}
\hline \hline SpT & CK79 $^{\mathrm{a}}$ & B $^{\mathrm{a}}$ & KH95 $^{\mathrm{a}}$ & C08 $^{\mathrm{a}}$ & R13 $^{\mathrm{a}}$ & L03 & Here \\
\hline F5 & $\ldots$ & $\ldots$ & 6440 & $\ldots$ & $\ldots$ & $\ldots$ & 6600 \\
F8 & $\ldots$ & $\ldots$ & 6200 & $\ldots$ & $\ldots$ & $\ldots$ & 6130 \\
G0 & 5902 & 6000 & 6030 & $\ldots$ & $\ldots$ & $\ldots$ & 5930 \\
G2 & 5768 & $\ldots$ & 5860 & $\ldots$ & $\ldots$ & $\ldots$ & 5690 \\
G5 & $\ldots$ & 5580 & 5770 & $\ldots$ & $\ldots$ & $\ldots$ & 5430 \\
G8 & 5445 & $\ldots$ & 5520 & $\ldots$ & $\ldots$ & $\ldots$ & 5180 \\
K0 & 5236 & $\ldots$ & 5250 & $\ldots$ & $\ldots$ & $\ldots$ & 4870 \\
K2 & 4954 & 5000 & 4900 & $\ldots$ & $\ldots$ & $\ldots$ & 4710 \\
K5 & 4395 & 4334 & 4350 & $\ldots$ & $\ldots$ & $\ldots$ & 4210 \\
K7 & 3999 & 4000 & 4060 & $\ldots$ & $\ldots$ & $\ldots$ & 4020 \\
M0 & 3917 & 3800 & 3850 & $\ldots$ & 3975 & $\ldots$ & 3900 \\
M1 & 3681 & 3650 & 3720 & 3608 & 3707 & 3705 & 3720 \\
M2 & 3499 & 3500 & 3580 & 3408 & 3529 & 3560 & 3560 \\
M3 & 3357 & 3350 & 3470 & 3208 & 3346 & 3415 & 3410 \\
M4 & 3228 & 3150 & 3370 & 3009 & 3166 & 3270 & 3190 \\
M5 & 3119 & 3000 & 3240 & 2809 & 2993 & 3125 & 2980 \\
M6 & $\ldots$ & $\ldots$ & 3050 & 2609 & 2834 & 2990 & 2860 \\
M7 & $\ldots$ & $\ldots$ & $\ldots$ & 2410 & 2697 & 2880 & 2770 \\
M8 & $\ldots$ & $\ldots$ & $\ldots$ & 2210 & 2588 & 2710 & 2670 \\
M9 & $\ldots$ & $\ldots$ & $\ldots$ & $\ldots$ & 2511 & 2400 & 2570 \\
\hline
\end{tabular}

Notes.

a Conversions developed for field dwarfs-CK: Cohen \& Kuhi (1979); B: Bessell (1979) and Bessell (1991); KH: Adopted by Kenyon \& Hartmann (1995); from Schmidt-Kaler (1982) and Straizys (1992); C08: Casagrande et al. (2008); R13: Rajpurohit et al. (2013); L03: Luhman et al. (2003).

dwarf temperature scales. ${ }^{6}$ Our scale matches the Luhman scale between M0-M4 and deviates at later spectral types. The differences between our scale and the Rajpurohit et al. (2013) scale are likely attributed to gravity differences between premain-sequence and dwarf stars. The K-dwarf temperature scale

\footnotetext{
6 The scales for Rajpurohit et al. (2013), Casagrande et al. (2008), and our work were calculated by using best-fit polynomials to the data points of spectral type versus effective temperature. For Casagrande et al. (2008), the
} data were obtained from tables of Rajpurohit et al. (2013).

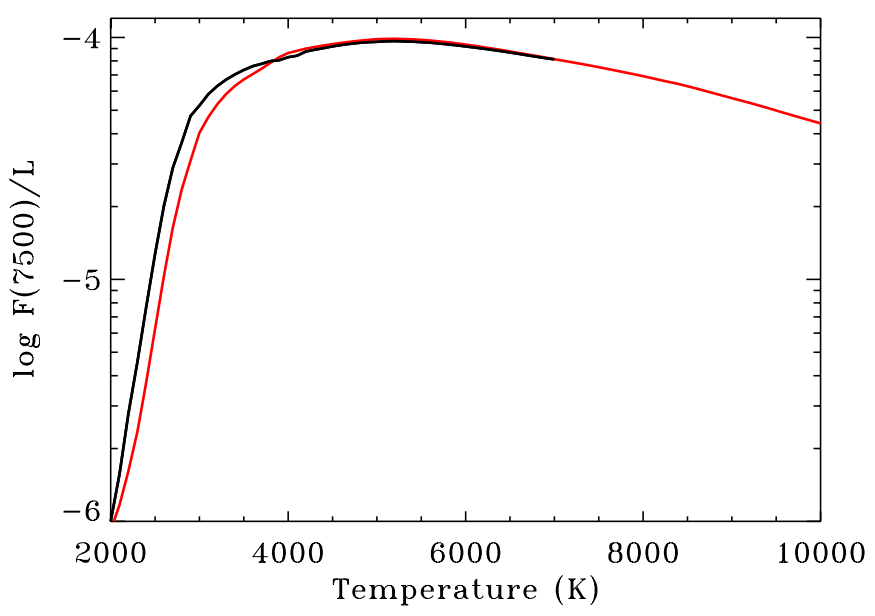

Figure 9. Bolometric correction applied to our data set, calculated by comparing the flux at $7510 \AA$ to the total luminosity in the BT-Settl stellar model (black, adopted for $T<7000 \mathrm{~K}$ ) and NextGen stellar model (red, adopted for $T>7000 \mathrm{~K}$ ). The bolometric correction is modified from the models to account for the weakness of the VO $7510 \AA$ absorption band, relative to model predictions.

(A color version of this figure is available in the online journal.)

Table 6

Bolometric Corrections from $F_{7510}$

\begin{tabular}{cccccc}
\hline \hline$T_{\text {phot }}$ & $F_{7510} / F_{\text {phot }}$ & $T_{\text {phot }}$ & $F_{7510} / F_{\text {phot }}$ & $T_{\text {phot }}$ & $F_{7510} / F_{\text {phot }}$ \\
\hline 2400 & $8.98 \mathrm{e}-06$ & 4300 & $8.90 \mathrm{e}-05$ & 6400 & $8.78 \mathrm{e}-05$ \\
2500 & $1.49 \mathrm{e}-05$ & 4400 & $9.04 \mathrm{e}-05$ & 6600 & $8.55 \mathrm{e}-05$ \\
2600 & $2.35 \mathrm{e}-05$ & 4500 & $9.18 \mathrm{e}-05$ & 6800 & $8.32 \mathrm{e}-05$ \\
2700 & $3.37 \mathrm{e}-05$ & 4600 & $9.31 \mathrm{e}-05$ & 7000 & $8.12 \mathrm{e}-05$ \\
2800 & $4.26 \mathrm{e}-05$ & 4700 & $9.41 \mathrm{e}-05$ & 7200 & $7.90 \mathrm{e}-05$ \\
2900 & $5.30 \mathrm{e}-05$ & 4800 & $9.50 \mathrm{e}-05$ & 7400 & $7.66 \mathrm{e}-05$ \\
3000 & $5.80 \mathrm{e}-05$ & 4900 & $9.56 \mathrm{e}-05$ & 7600 & $7.41 \mathrm{e}-05$ \\
3100 & $6.41 \mathrm{e}-05$ & 5000 & $9.59 \mathrm{e}-05$ & 7800 & $7.17 \mathrm{e}-05$ \\
3200 & $6.98 \mathrm{e}-05$ & 5100 & $9.64 \mathrm{e}-05$ & 8000 & $6.92 \mathrm{e}-05$ \\
3300 & $7.52 \mathrm{e}-05$ & 5200 & $9.66 \mathrm{e}-05$ & 8200 & $6.66 \mathrm{e}-05$ \\
3400 & $7.93 \mathrm{e}-05$ & 5300 & $9.64 \mathrm{e}-05$ & 8400 & $6.42 \mathrm{e}-05$ \\
3500 & $8.20 \mathrm{e}-05$ & 5400 & $9.61 \mathrm{e}-05$ & 8600 & $6.15 \mathrm{e}-05$ \\
3600 & $8.43 \mathrm{e}-05$ & 5500 & $9.56 \mathrm{e}-05$ & 8800 & $5.88 \mathrm{e}-05$ \\
3700 & $8.58 \mathrm{e}-05$ & 5600 & $9.51 \mathrm{e}-05$ & 9000 & $5.61 \mathrm{e}-05$ \\
3800 & $8.73 \mathrm{e}-05$ & 5700 & $9.44 \mathrm{e}-05$ & 9200 & $5.37 \mathrm{e}-05$ \\
3900 & $8.80 \mathrm{e}-05$ & 5800 & $9.36 \mathrm{e}-05$ & 9400 & $5.12 \mathrm{e}-05$ \\
4000 & $8.89 \mathrm{e}-05$ & 5900 & $9.28 \mathrm{e}-05$ & 9600 & $4.86 \mathrm{e}-05$ \\
4100 & $8.83 \mathrm{e}-05$ & 6000 & $9.18 \mathrm{e}-05$ & 9800 & $4.63 \mathrm{e}-05$ \\
4200 & $8.75 \mathrm{e}-05$ & 6200 & $8.99 \mathrm{e}-05$ & 10000 & $4.41 \mathrm{e}-05$ \\
\hline
\end{tabular}

Notes. $T<7000 \mathrm{~K}$ from BT-Settl models, corrected for scaling factor listed in Table 13. $T>7000 \mathrm{~K}$ from Phoenix models.

is shifted to lower temperatures relative to the scale used by Kenyon \& Hartmann (1995).

\subsubsection{Photospheric Luminosities}

Stellar photospheric luminosities, $L_{\text {phot }}$, are calculated using the Phoenix/BT-Settl models with CFITSIO opacities (Allard et al. 2012) for effective temperatures $<7000$ K. Similar bolometric corrections for hotter stars are calculated from the NextGen model spectra (Hauschildt et al. 1999). The flux ratio $F_{7510} / L_{\text {phot }}$ is used as the bolometric correction and applied to the measured photospheric fluxes (Figure 9 and Table 6). These conversions all assume a gravity of $\log g=4$. Differences due to subtracting the a flat continuum in late $M$ dwarfs (Section 3.2) amount to $\sim 1 \%$ of the total stellar luminosity and are ignored here. 
At temperatures $<3500 \mathrm{~K}$, the opacity in a $\mathrm{VO}$ absorption band at $7500 \AA$ is much larger in synthetic spectra than in the observed spectra. At these temperatures, the bolometric correction for $7510 \AA$ flux is calculated by fitting a line between the flux at 7300 and $7580 \AA$, omitting the VO opacity from the fit.

\subsection{Including the Accretion Continuum in Spectral Fits}

Many young stars have strong enough accretion to partially or, in rare cases, even fully mask the photosphere. Fully masked stars have no detectable photosphere and have no measured spectral type, but an extinction is still measurable with an estimate for the shape of the accretion continuum. Even for lightly veiled stars, the extinction should be measured by comparing the observed colors to a combination of the photospheric spectrum and the accretion continuum spectrum. This subsection describes how to estimate the shape and strength of the accretion continuum so that it can be included in extinction measurements.

\subsubsection{The Shape of the Accretion Continuum Spectrum}

Including the accretion spectrum into the spectral fit requires an estimate for veiling versus wavelength. The measureable accretion continuum is produced by $\mathrm{H}$ recombination to the $n=2$ level (Balmer continuum, $\lambda<3700 \AA$ ) and to the $n=3$ level (Paschen continuum $\lambda<8200 \AA$ ), plus an $\mathrm{H}^{-}$ continuum (for detailed descriptions, see Calvet \& Hartmann 1992; Calvet \& Gullbring 1998). The ratio of these different components depends on the temperature, density, and optical depth of the accreting gas and heated chromosphere. The size of the Balmer jump between stars is different (e.g., Herczeg et al. 2009), which forces this analysis to be restricted to the shape of the continuum either at $<3700 \AA$ or between $3700-8000 \AA$. Here we concentrate on the emission at $>4000 \AA$. The spectral slope at $<3700 \AA$ is uncertain in the observed spectra due to the large wavelength dependence in the telluric correction near the atmospheric cutoff.

The exact shape of the accretion continuum likely depends on the properties of the accretion flows. Models of the accretion continuum typically assume a single shock structure. Fitting the Balmer continuum emission leads to model spectra where at $>4000 \AA$, the flux decreases with increasing wavelength (see Figure 3 from Ingleby et al. 2013 for spectra from isothermal models at different densities). These synthetic spectra underestimate the observed veiling at red wavelengths (see, e.g., models of Calvet \& Gullbring 1998 and measurements by Basri \& Batalha 1990 and Fischer et al. 2011). Ingleby et al. (2013) explains this problem by invoking the presence of accretion columns with a range of densities, some of which are lower density than has been typically assumed and produces cooler accretion shocks. This physical situation may be expected if accretion occurs in several different flows or if a single flow has a range of densities, perhaps because the magnetic field connects with the disk at a range of radii. The weaker shocks yield cooler temperatures and produce redder emission, thereby recovering the measured veilings around $1 \mu \mathrm{m}$.

Empirical measurements of the accretion continuum flux are shown in Figure 10. The fraction of emission attributed to the accretion continuum is calculated from the optical veiling measurements of Fischer et al. (2011) and the relationship $\left(r_{\lambda}=\right.$ $\left.\left(F_{\text {acc }} / F_{\text {phot }}\right)\right)$. This fraction is then converted to the accretion continuum flux by two different methods: (a) multiplying the fraction by our flux-calibrated spectra that are corrected for extinction and (b) multiplying the veiling by the flux from the

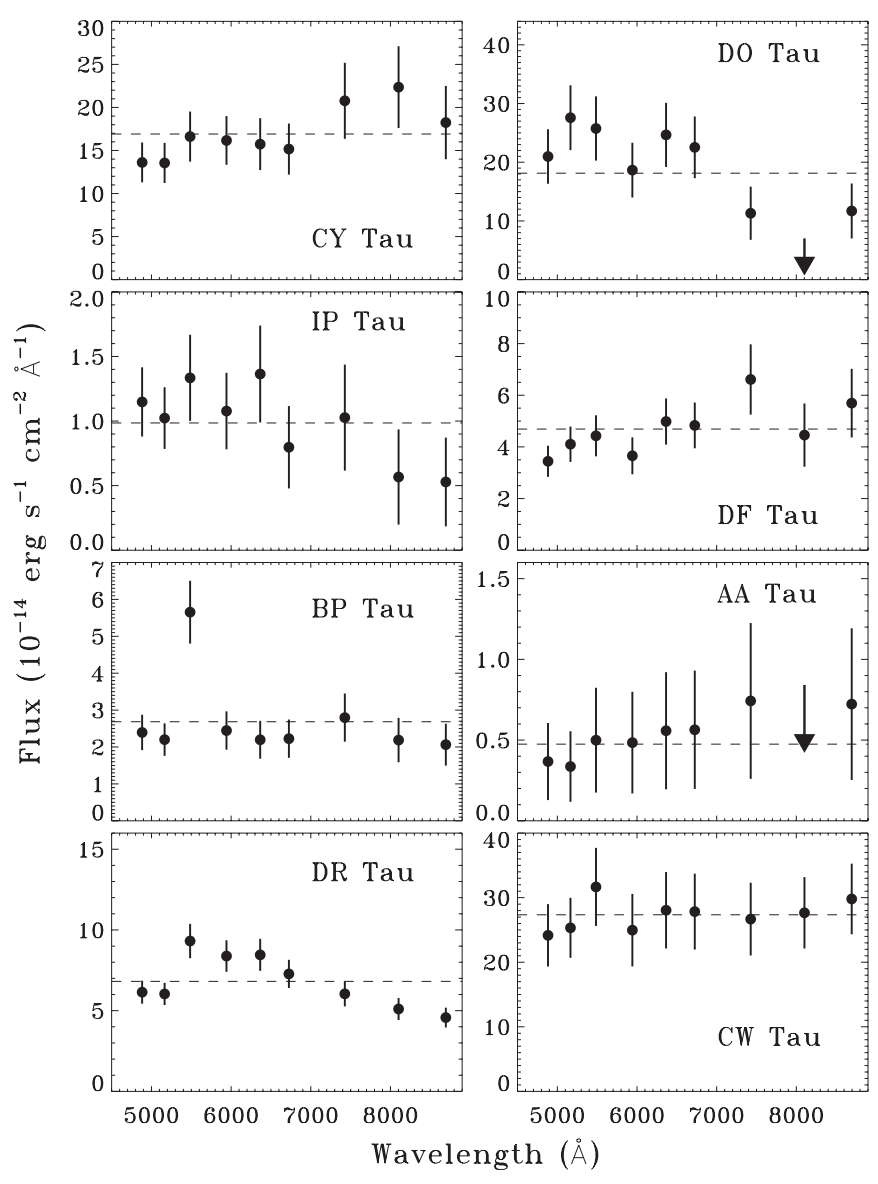

Figure 10. Accretion continuum flux of eight stars. For the top six stars, the accretion continuum is calculated from veiling measurements in Fischer et al. (2011) and the stellar flux measured here and then corrected for extinction (method b in the text). For the heavily veiled stars DR Tau and CW Tau in the bottom panel, the accretion continuum is calculated by multiplying the measured veiling to photospheric templates (method a). Uncertainties are assessed by including a 0.05 uncertainty in the veiling measurement and a $10 \%$ uncertainty in the flux. Differences in the slope of the accretion continuum between sources (e.g., slightly rising with wavelength for AA Tau but slightly falling for IP Tau) may be real or may be introduced by observational uncertainties. Our assumption that the optical accretion continuum is flat (shown here as the dashed horizontal line) is roughly consistent with these calculations and with the optical veiling measurements of Basri \& Batalha (1990).

template spectrum for the relevant spectral type from Table 4 . Uncertainties in the accretion continuum are estimated to be 0.05 times the flux from the calibrated spectrum and 0.05 times the total standard+accretion flux, respectively. ${ }^{7}$ These two methods are somewhat independent from each other but yield similar results.

Table 7 compares the $\chi^{2}$ from linear fits $\left(\chi_{\text {line }}^{2}\right)$ and flat fits $\left(\chi_{\text {flat }}^{2}\right)$ to the accretion continuum fluxes for methods (a) veil*flux and (b) veil*template described above. Most cases are consistent with a flat accretion continuum. The veiling measurements tend to be smaller at longer wavelengths because the photospheric flux is brighter in the red than in the blue. This exercise presents somewhat circular logic because the spectral type and extinction are both calculated assuming that the accretion continuum is flat. Method (b) depends less on the assumption of a flat continuum

\footnotetext{
7 The uncertainty in veiling measurements is typically $0.05-0.1$ for moderately veiled stars and much larger for heavily veiled stars because the definition is the accretion flux divided by the photospheric flux. In either case, the uncertainty in the flux is $5 \%-10 \%$ of the total observed flux and not $5 \%-10 \%$ of the flux attributed to the accretion continuum.
} 
Table 7

Slopes of the Accretion Continuum

\begin{tabular}{|c|c|c|c|c|c|c|}
\hline \multirow[t]{2}{*}{ Star } & \multicolumn{3}{|c|}{ a: veil * flux } & \multicolumn{3}{|c|}{ b: veil * template } \\
\hline & $F 4 / F 8^{\mathrm{a}}$ & $\chi_{\text {line }}^{2}$ & $\chi_{\text {flat }}^{2}$ & $F 4 / F 8^{\mathrm{a}}$ & $\chi_{\text {line }}^{2}$ & $\chi_{\text {flat }}^{2}$ \\
\hline AA Tau & 0.79 & 0.4 & 0.4 & 0.64 & 0.5 & 0.4 \\
\hline BP Tau & 1.09 & 0.4 & 0.5 & 0.70 & 3.7 & 1.4 \\
\hline CW Tau ${ }^{b}$ & 1.03 & 1.4 & 1.6 & 1.03 & 0.8 & 0.9 \\
\hline $\mathrm{CW} \mathrm{Tau}{ }^{\mathrm{b}}$ & 1.08 & 1.1 & 1.2 & 1.08 & 0.5 & 0.5 \\
\hline CY Tau & 1.39 & 4.3 & 5.7 & 1.00 & 8.7 & 10 \\
\hline DF Tau & 0.53 & 17 & 6.8 & 0.3 & 61 & 7.4 \\
\hline DO Tau & 1.29 & 1.7 & 0.8 & 0.76 & 8.9 & 5.2 \\
\hline IP Tau & 1.59 & 1.7 & 1.9 & 1.46 & 1.3 & 2.0 \\
\hline DG Tau & 1.04 & 2.0 & 2.3 & 0.80 & 3.7 & 2.1 \\
\hline DK Tau & 0.87 & 2.2 & 2.1 & 0.72 & 3.2 & 1.4 \\
\hline DL Tau & 0.98 & 4.2 & 4.7 & 0.98 & 1.4 & 1.6 \\
\hline DR Tau & 0.83 & 12.6 & 10 & 1.42 & 3.9 & 0.9 \\
\hline HN Tau A & 1.42 & 3.9 & 0.9 & 1.46 & 5.5 & 1.8 \\
\hline
\end{tabular}

Notes. Spectral slopes of the accretion continuum for two methods. of converting the veiling to flux, see also Figure 10. Flat $\chi^{2}$ has 8 degrees of freedom, linear fit has 7 .

a F4/F8: flux ratio of accretion continuum at $4000 \AA$ to $8000 \AA$.

${ }^{\mathrm{b}}$ Fischer et al. (2011) observed CW Tau twice.

but is sensitive to gravity and spectral type uncertainties in the optical colors. However, the results from both approaches demonstrate that the assumption of a flat accretion continuum is reasonable and self-consistent.

Based on these calculations and the results of Ingleby et al. (2013), we make the simplifying approximations that the shape of the accretion continuum is (1) the same for all accretors and (2) that the accretion continuum is constant, in erg cm $\mathrm{cm}^{-2} \mathrm{~s}^{-1} \AA^{-1}$, at optical wavelengths. In contrast, Hartigan \& Kenyon (2003) assumes that the accretion continuum is a line with a slope that differs from star to star. Real differences between spectra are surely missed in our approach. However, our approach is simpler and reproduces the observed spectra with fewer free parameters. A more rigorous assessment of the accretion continuum spectrum is possible from broadband high resolution spectra, which has been applied to small samples (e.g., Fischer et al. 2011; McClure et al. 2013) but is time consuming, has not yet been implemented for large data sets, and suffers from the same degeneracies and systematic trades between surface gravity, reddening, and veiling by emission from the accretion shock and the warm inner disk.

\subsubsection{Veiling Estimates at Ca I 4227}

Veiling can be accurately measured from high resolution spectroscopy (e.g., Basri \& Batalha 1990; Hartigan et al. 1991) or estimated from low resolution spectrophotometric fitting (e.g., Fischer et al. 2011; Ingleby et al. 2013). Here we develop an intermediate approach to measure the veiling by comparing the depth of a single, strong absorption line to its depth in a template star. While accurate veiling measurements require high resolution spectroscopy, veiling may be estimated by measuring the depth of strong photospheric lines in low resolution spectra. This section concentrates on the strong Ca I $\lambda 4227$ line.

Figure 11 shows spectra of the CaI region versus spectral type. The Ca I equivalent width depends on spectral type as

$$
\mathrm{EW}(\mathrm{CaI})=-189.218+7.36 x-0.072 x^{2},
$$

where $x=50,58,63$ for K0, M0, and M5, respectively (Figure 12). Values lower than this equivalent width indicate

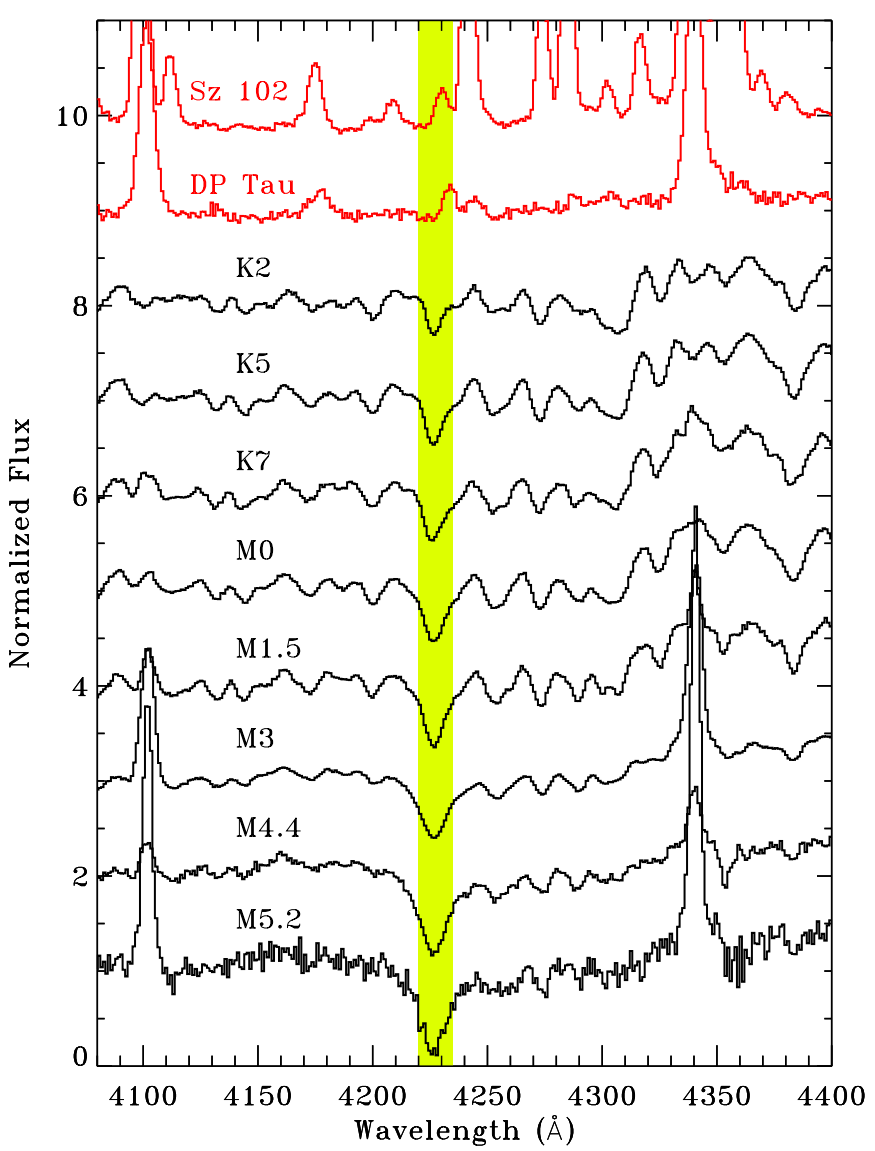

Figure 11. Ca I $\lambda 4227$ photospheric absorption line (shaded yellow region) vs. spectral type for WTTSs from early K through mid M for stars listed in Table 4. In low-resolution spectra, emission lines produced by accretion processes can fill in the photospheric absorption, as shown for heavily veiled spectra of $\mathrm{Sz} 102$ and DP Tau (top red spectra).

(A color version of this figure is available in the online journal.)

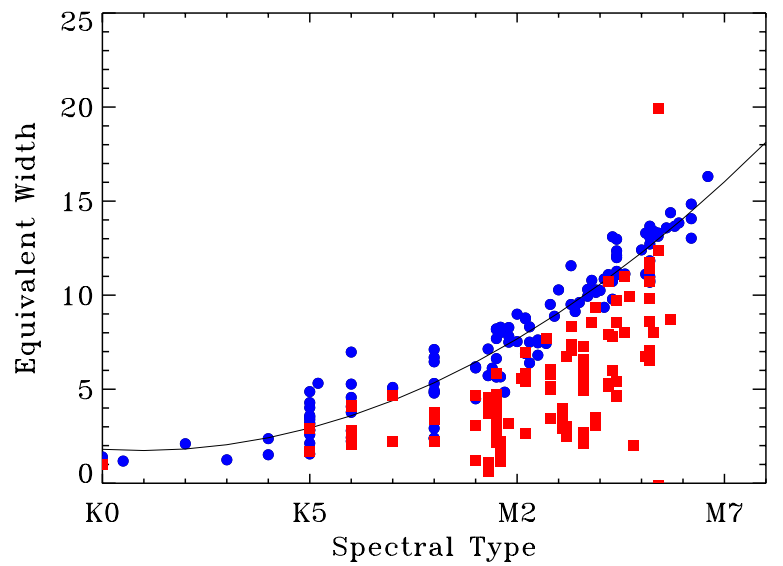

Figure 12. Ca I $\lambda 4227$ line equivalent width vs. SpT, for WTTSs (blue circles) and CTTSs (red squares) in our sample. The accretion continuum at $4227 \AA$ is calculated by comparing the equivalent width in the line to that expected at a given SpT. For CTTSs, this line is often shallower than expected because of accretion, a fact which we exploit to measure the strength of the accretion continuum from low-resolution spectra.

(A color version of this figure is available in the online journal.)

that the depth of the photospheric absorption is reduced because of extra emission from the accretion continuum. This difference yields the strength of the accretion continuum at $4227 \AA$. 


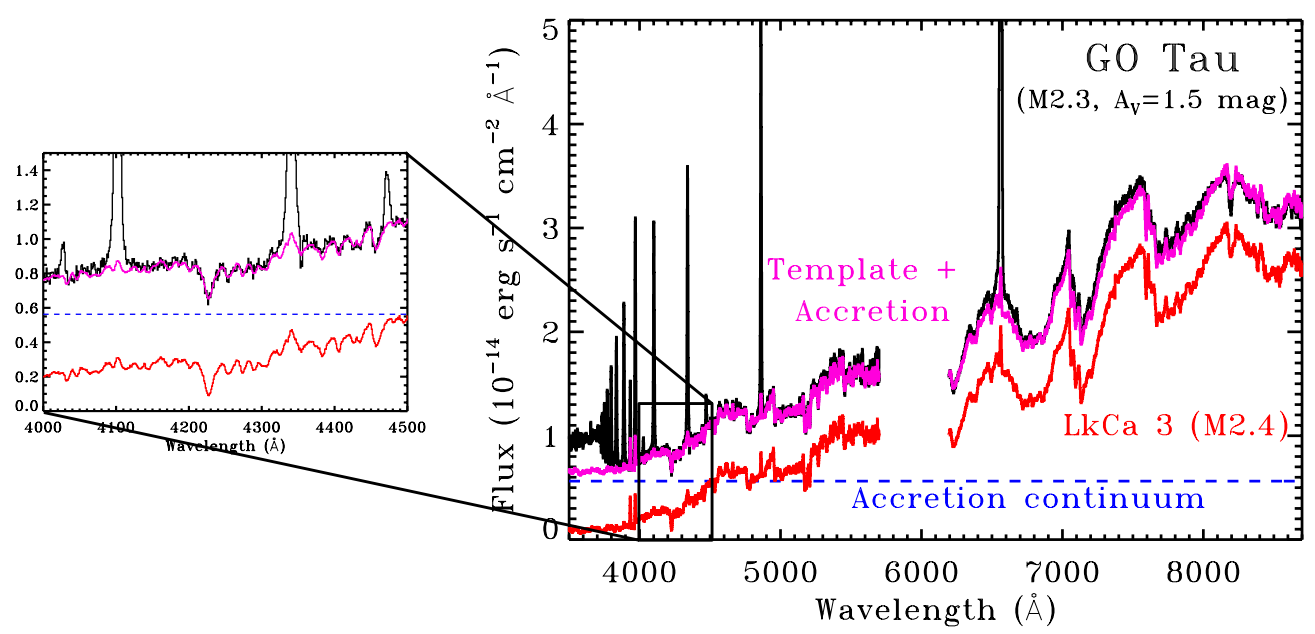

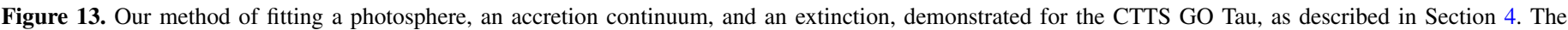

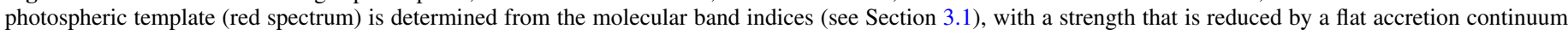

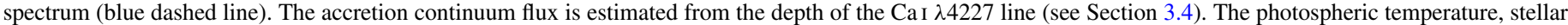
luminosity, accretion continuum strength, and extinction are all free parameters that vary until a best fit (purple spectrum) is found and visually confirmed.

(A color version of this figure is available in the online journal.)

Figure 13 shows an example of how the veiling at $4227 \AA$ is estimated for each source. A flat accretion spectrum is added to the template photospheric spectrum so that the combination matches the observed line depth. The CaI absorption line is sometimes filled in by emission from nearby Fe lines, thereby affecting the veiling estimate (Gahm et al. 2008; Petrov et al. 2011; Dodin \& Lamzin 2012). Although this particular line is also sensitive to surface gravity, the use of temperature matched WTTS as templates should mitigate gravity-dependent line depth systematics. For cooler stars, calculating the accretion continuum flux at $4227 \AA$ maximizes the sensitivity to accretion for cool stars because the ratio of accretion flux to photospheric flux is higher at short wavelengths.

\section{FINAL ASSESSMENT OF STELLAR PARAMETERS}

The previous section provides a grid of spectral templates (Table 4), a method to estimate the strength of the accretion continuum emission, and a description of extinction. In this section, we apply these analysis tools to simultaneously measure the spectral type, extinction, and accretion for our sample. Our procedures for $\mathrm{K}$ and $\mathrm{M}$ spectra with zero to moderate veiling are discussed in Section 4.1. Heavily veiled stars require a different approach and are discussed separately in Section 4.2. We then describe in Section 4.3 how these methods are implemented for several selected stars. In Sections 4.4 and 4.5, spectral types and extinctions are compared to selected measurements in the literature.

Our final spectral types, extinctions, veilings, and photospheric parameters are presented in Appendix C. Some stars have extinction values that are measured to be negative. These extinctions are retained for statistical comparisons to other studies but are unphysical and treated as zero extinction when calculating luminosities.

\subsection{Stars with Zero to Moderate Veiling}

A best-fit $\mathrm{SpT}, L_{\mathrm{phot}}, A_{V}$, and accretion continuum flux (veiling) is calculated for each star by fitting 15 different wavelength regions from 4400-8600 $\AA$. The wavelength regions are selected by concentrating on obtaining photospheric flux measurements both within and outside of absorption bands. For stars with spectra covered by emission lines (more than the $\mathrm{H}$ Balmer, $\mathrm{He}$ I, and $\mathrm{Ca}$ II lines), the bluest regions are excised from the fit and the remaining wavelength regions are altered to focus on continuum regions. The wavelength regions incorporate the spectral type indices described previously. The accretion continuum flux is initially estimated from the equivalent width of the $\mathrm{Ca}$ I line and is manually adjusted. All fits are confirmed by eye. This approach is similar to that taken by Hartigan \& Kenyon (2003) to analyze spectra of close binaries in Taurus, although our spectral coverage is broader and our grid of WTTSs is more complete.

Spectral types and extinctions are calculated from the spectral grid established in Section 3.3. The spectral types are listed to $1,0.5$, and 0.1 subclasses for spectral types earlier than $\mathrm{K} 5$, K5-M0, and later than M0. Extinction is calculated at intervals of $A_{V}=0.02 \mathrm{mag}$. and listed to the closest $0.05 \mathrm{mag}$. For M dwarfs, these values approximately Nyquist sample the uncertainties of $\sim 0.2-0.3$ subclasses in SpT and $\sim 0.2-0.3 \mathrm{mag}$. in $A_{V}$. The accretion continuum is fixed to 0 for stars with no obvious signs of accretion. For accreting stars, the accretion continuum is also initially a free parameter. Comparing the results of this grid yield an initial best fit to the spectral type, accretion continuum strength, and extinction. This initial spectral type measurement is then used to constrain the accretion continuum from fitting to the Ca I $4227 \AA$ line (Section 3.4). With this new accretion continuum, a new best-fit spectral type and extinction are calculated. For stars used as templates, our best fit $\mathrm{SpT}$ and $A_{V}$ are calculated here from the full photospheric grid and may therefore differ slightly from the values listed in Table 4.

As a check for self-consistency, most of our final SpT and $A_{V}$ agree with earlier measurements in this project to 0.1 subclass in SpT and 0.1 mag., respectively, for M dwarfs. The previous measurements were based on a slightly different spectral grid. This comparison defines our internal precision for extinction and spectral type.

Figure 13 demonstrates how this method is implemented for the CTTS GO Tau. The accretion continuum and photospheric spectrum together match the Ca I $4227 \AA$ line, many other bumps in the blue spectrum, and the $\mathrm{TiO}$ absorption in the red spectrum. In some cases the best fit accretion continuum is found to differ 


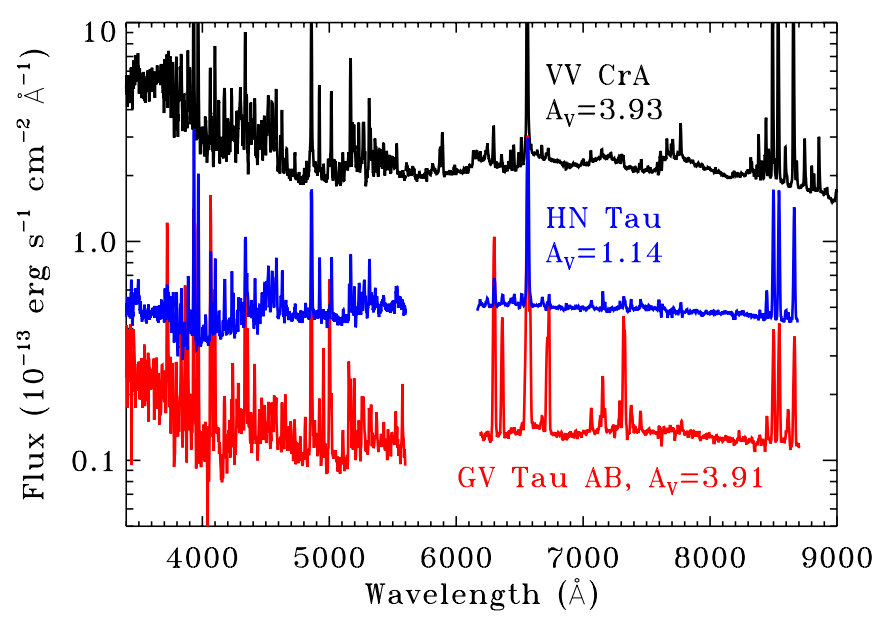

Figure 14. Extinction-corrected spectra of heavily veiled stars. These stars cannot be spectral typed from low-resolution spectra and are classified as "continuum" objects. Extinctions for heavily veiled sources are measured by assuming that the observed continuum is flat. In our low-resolution spectra, this continuum is measurable at $>4600 \AA$. The blue spectra of heavily veiled objects are typically covered in a forest of emission lines.

(A color version of this figure is available in the online journal.)

from the Ca I absorption depth calculated based on Figure 12, likely because weak line emission fills in the photospheric absorption line. Indeed, emission in several lines around $4227 \AA$ is seen from many heavily veiled stars. Accounting for veiling is particularly important for stars with moderate or heavy veiling (>0.1 at $7000 \AA$ ), as described for several individual sources in Section 4.2. Even for lightly veiled stars, accretion can affect the $\mathrm{SpT}$ and extinction.

Repeated observations for 59 targets in our sample yield more accurate photospheric measurements because the best fit spectral type should be consistent despite changes in the veiling. The spectral type, extinction, and accretion continuum flux were initially left as free parameters for each spectrum. No significant change in SpT was detected. The final SpT was set to the average SpT of all spectra of the object. The spectra were then fit again with this SpT, leaving extinction and the accretion continuum flux as the free parameters. The average extinction value was then applied to all observations of a given object, when possible, to calculate the accretion and photospheric luminosity. In three cases, definitive changes are detected and a single extinction cannot be applied. This approach of trying to find a single extinction to explain repeated spectra should miss some real changes in extinction. Changes in the strength of the accretion continuum are frequently detected among the different spectra, usually with amplitude changes of less than a factor of $\sim$ three. No star in our sample changes between lightly and heavily veiled. Spectral variability will be discussed in detail in a subsequent paper.

\subsection{Heavily Veiled Stars}

Heavily veiled stars have spectra dominated by emission produced by accretion, with little or no detectable photospheric component and a forest of emission lines at blue wavelengths (Figure 14). In some cases, high resolution spectra can yield enough photospheric absorption lines to reveal a spectral type (White \& Hillenbrand 2004). In our work, the $5200 \AA$ band and the $\mathrm{TiO}$ bands can be detected from some objects despite high veiling. RNO 91 has few photospheric features but many fewer emission lines than the other stars. A weak $5200 \AA$ bump of
RNO 91 suggests a SpT of K0-K2, while a stronger bump for HN Tau A suggests K2-K5. Both RNO 91 and HN Tau A lack detectable TiO absorption. DL Tau has weak TiO absorption and has a SpT between K5-K8. Two cases, CW Tau and DG Tau, are assigned spectral types of $\mathrm{K} 3$ and $\mathrm{K} 6.5$ and are discussed in detail in Sections 4.3.5 and 4.3.6.

The stars VV CrA, GV Tau AB, AS 205A, and Sz 102 have no obvious photospheric features in our spectra and are listed here as continuum sources. These sources likely have spectral types between late $\mathrm{G}$ and early $\mathrm{M}$, since earlier and later spectral types are unlikely based on indirect arguments. At spectral types earlier than late $G$, the photosphere is bright enough that it dominates the spectrum for reasonable accretion rates. For stars cooler than early $\mathrm{M}$, the photosphere is so red that the TiO bands are always easily detected at red wavelengths (e.g., Herczeg \& Hillenbrand 2008). An M5 star with veiling of 30 at $8400 \AA$ would still have a $\mathrm{TiO}$ band depth of $3 \%$, which is detectable in most of our spectra because of sufficient signal to noise and flux calibration accuracy. Such a high veiling is not expected for M5 stars because the accretion rate correlates with $M_{*}^{2}$. Indeed, several mid-late $M$ dwarfs (e.g., GM Tau, CIDA 1, 2MASS J04141188+2811535, J04414825+2534304) have blue spectra with high veiling and are covered by a forest of chromospheric emission lines, similar to the cases of CW Tau and DG Tau, but they have red spectra with easily identified $\mathrm{TiO}$ absorption. Only between $\mathrm{K} 0-\mathrm{M} 2$ are photospheric features weak enough and the photosphere faint enough that it could be fully masked by a strong accretion continuum. ${ }^{8}$

For heavily veiled stars, the extinction is calculated by assuming that the accretion continuum is flat (see CW Tau and DR Tau in Figure 10) and dominates the optical emission. Extinction corrected spectra for three stars are presented in Figure 14. Fits to the continuum were made to avoid emission lines and $\mathrm{TiO}$ emission (see Section 5.4.2). The extinction is likely underestimated to stars such as HL Tau, Sz 102, GV Tau, and 2MASS J04381486+2611399 because of edge-on disks and/or remnant envelopes. The optical flux from these sources is very faint but appears to have no or little extinction. These three objects also have forbidden emission lines with large equivalent widths, characteristic of sources where the edge-on disk occults the star but not the outflow.

\subsection{Examples of Specific Stars}

These subsections illustrate how the logic described above is implemented for several example stars, which cover a range of spectral type and accretion rate. The pre-main-sequence tracks applied here to calculate masses and ages are combined from Tognelli et al. (2011) and Baraffe et al. (2003), as described in Appendix C.

\subsubsection{UScoCTIO 33}

UScoCTIO 33 was originally identified as a possible member of the Upper Scorpius OB Association in a photometric survey by Ardila et al. (2000). A spectroscopic survey by Preibisch et al. (2002) confirmed membership, classified the star as M3, and found strong $\mathrm{H} \alpha$ emission indicative of accretion.

\footnotetext{
8 These arguments do not apply at the earliest stages of protostellar evolution or for outbursts, when accretion rates are much higher than those typically measured in the T Tauri phase. In these cases, the accretion luminosity may be much brighter than any photospheric luminosity, regardless of the underlying spectral type on an unheated photosphere, if present.
} 


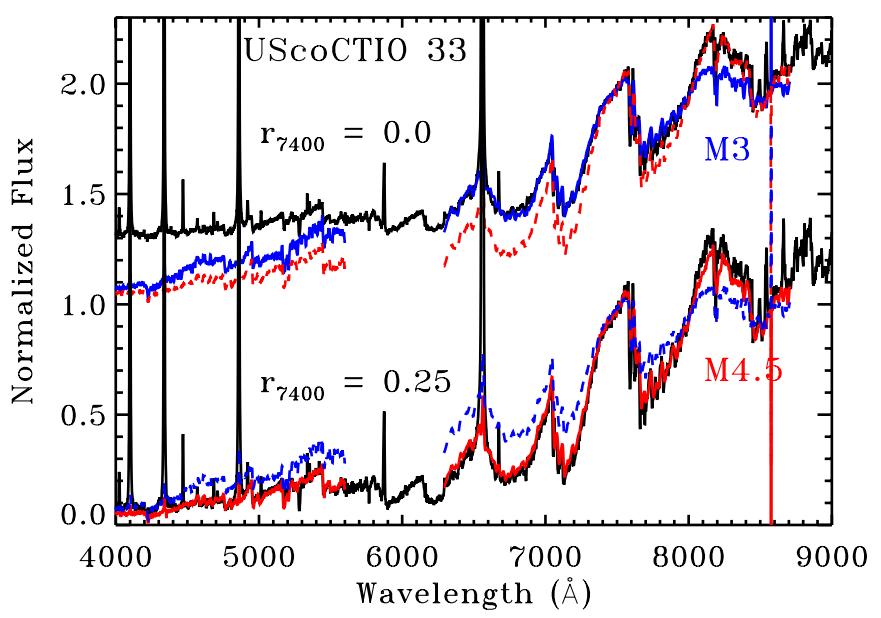

Figure 15. Spectrum of UScoCTIO 33 compared to M3 and M4.5 spectra with veiling $r_{7510}=0.0$ (top) and 0.25 (bottom). If veiling by accretion is not accounted for, the 6000-9000 ̊ spectrum appears to be an M3 (top). However, the M3 template badly underestimates the blue emission. If we estimate and subtract off an accretion continuum, the spectrum is consistent with an M4.5 star (bottom).

(A color version of this figure is available in the online journal.)

Figure 15 shows our Keck spectrum of UScoCTIO 33 compared with M3 and M4.5 stars with a veiling $r_{7525}=0.0$ and 0.25 . If the veiling is 0 , the red spectrum is best classified as an M3 spectral type, with only small inconsistencies between the template and the spectrum. However, the M3 template spectrum is much weaker than the observed blue emission. The veiling $r_{7525}=0.25$ is calculated from the depth of the Ca I $\lambda 4227$ line. Subtracting this accretion continuum off of the observed spectrum yields photospheric lines that are deeper than the uncorrected observation. The consequent M4.5 spectral type with veiling improves the fit to the red and blue spectra.

The M4.5 SpT leads to a mass of $0.11 M_{\odot}$ and an age of 5 Myr. The M3 SpT and no veiling yields a mass of $0.32 M_{\odot}$ and an age of $35 \mathrm{Myr}$, assuming no change in $A_{V}$.

\subsubsection{DM Tau}

The literature spectral type of M1 for DM Tau traces back to Cohen \& Kuhi (1979). Despite significant interest as the host of a transition disk (e.g., Calvet et al. 2005), its spectral type has not been reassessed using modern techniques.

Figure 16 shows the veiling-corrected DM Tau spectrum 2008 December 29, compared with M2, M3, and M4 spectra. The veiling is calculated from the depth of the Ca I $4227 \AA$ line. The veiling $r_{7510}=0.17$ leads to SpT of M3 and $A_{V}=0.08$. If the composite photospheric+accretion spectrum is not constrained by a good fit to the $\mathrm{Ca}$ I $\lambda 4227$ line, then $r_{7510}$ could range from 0.09, with SpT M2.7 and $A_{V}=-0.20$, to 0.31, with SpT M3.4 and $A_{V}=0.50$. In this case, the extinction increases with later spectral type because the veiling has increased (see also the case of DP Tau). If the blue side is ignored entirely, then a veiling of $r_{7510}=0$ would yield M2.5 and $A_{V}=0.06$ while an upper limit on veiling of $r_{7510}=0.39$ would yield M4.1 and $A_{V}=-0.06$. In these latter cases, the resulting red spectrum looks reasonable. The uncertainties in SpT and veiling are about half the size of these ranges when using the blue and red spectra together. Even with the blue+red spectrum, differences between M2 and M4 are subtle and are likely undetectable with a cruder method, such as photometry.

The change from M1 to M3 for DM Tau leads to a younger age (17 versus $4.9 \mathrm{Myr}$ ) and a lower mass $\left(0.62\right.$ versus $\left.0.35 M_{\odot}\right)$, assuming no change in $A_{V}$. The luminosity does not change significantly because the bolometric correction from the red photospheric flux is similar for an M1 and an M3 star.

\subsubsection{TW Hya}

Despite being the closest and possibly the most studied CTTS, the spectral type of TW Hya has been the subject of some controversy. The original spectral type of K7 was obtained from low resolution spectroscopy by de la Reza et al. (1989). Yang et al. (2005) used high-resolution optical spectra to measure an effective temperature of $4126 \pm 24 \mathrm{~K}$, equivalent to K6.5.
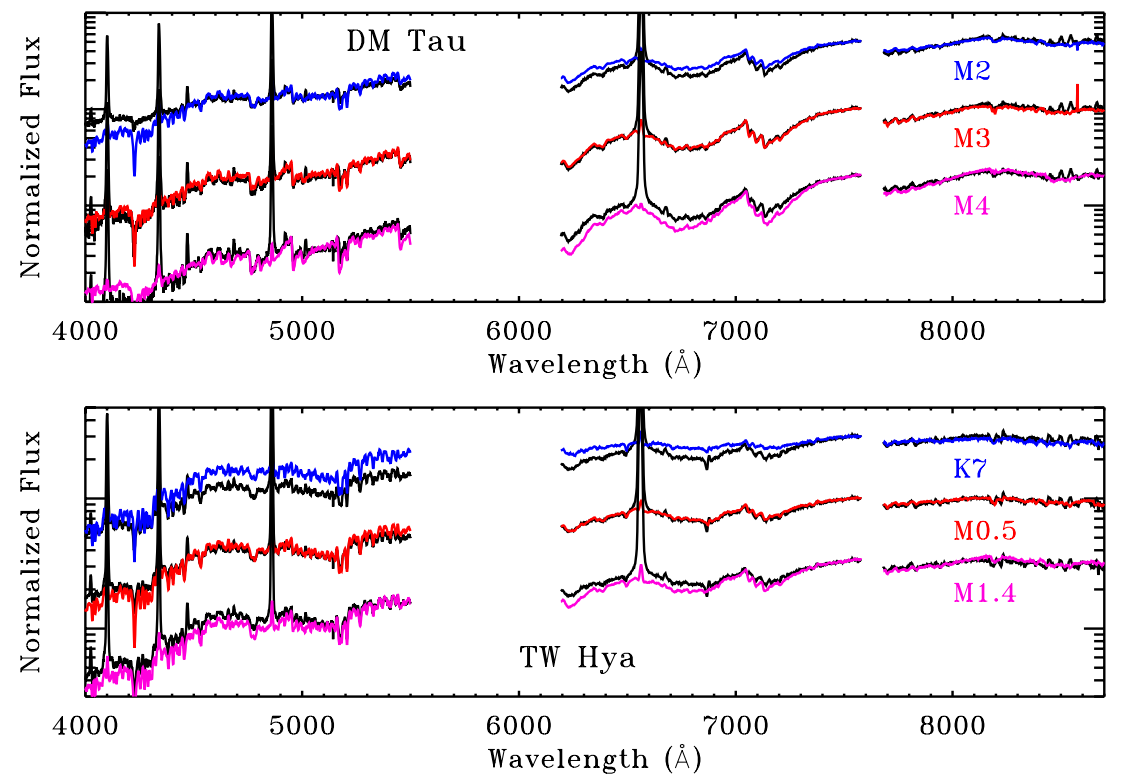

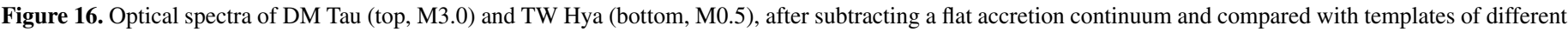

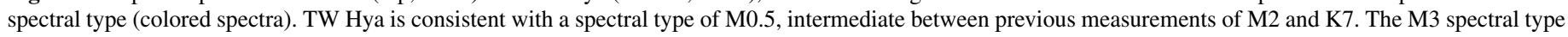
of DM Tau is later than literature measurements of M1.

(A color version of this figure is available in the online journal.) 

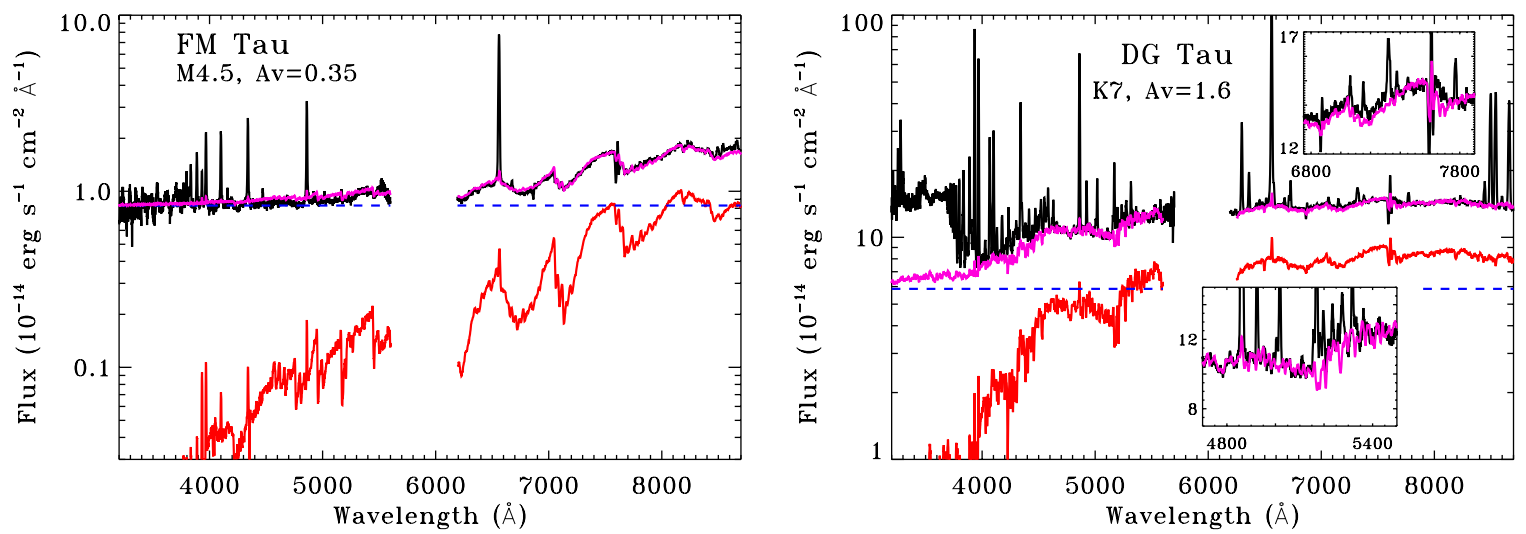

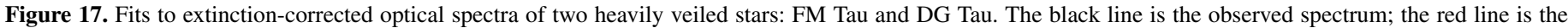
photospheric template; the dashed blue line shows the flat accretion continuum; and the purple line shows the best-fit template plus accretion continuum.

(A color version of this figure is available in the online journal.)

They caution that the uncertainty in effective temperature likely underestimates systematic uncertainties. This spectral type is consistent with the K7 SpT derived by Alencar \& Batalha (2002), also from high-resolution spectra. In contrast, Vacca \& Sandell (2011) relied on low resolution spectra from 1-2.4 $\mu \mathrm{m}$ to obtain a new spectral type of M2.5. McClure et al. (2013) found that TW Hya is consistent with roughly M0 spectral type at $1.1 \mu \mathrm{m}$. Debes et al. (2013) argued that the 5500-10200 spectrum is a composite $\mathrm{K} 7+\mathrm{M} 2$ in the near-IR, with the warmer component related to accretion. Debes et al. (2013) did not consider veiling by the accretion continuum, which would preferentially cause the measured SpT at short wavelengths to be earlier than the actual spectral type.

Figure 16 shows that the optical spectrum is consistent with an M0.5 spectral type, which corresponds to $\sim 3810 \mathrm{~K}$, with $A_{V}=0.0 \mathrm{mag}$. and veilings that range $r=0.09-0.21$. This spectral type is consistent with all of our TW Hya spectra, obtained on seven different nights in 2008 January, May, and December. The effective temperature is significantly lower than that from Alencar \& Batalha (2002) and Yang et al. (2005). While spectral fits to high resolution spectra may suffer from emission lines filling in photospheric absorption for strong accretors (e.g., Gahm et al. 2008; Dodin \& Lamzin 2012), this problem is not expected to be significant for a weakly accreting star like TW Hya. However, the high veiling during the Yang et al. (2005) observation, three times higher than the median veiling measured here and by Alencar \& Batalha (2002), may have complicated their temperature measurements.

Our spectral type is inconsistent with the late spectral type of Vacca \& Sandell (2011). An M2 spectral type could only be recovered for our TW Hya spectra if the accretion continuum were three times larger in the red than that measured in the blue, which is inconsistent with both models and previous measurements of the accretion continuum. The spectral templates of Vacca \& Sandell (2011) were dwarf stars, which may differ in certain near-IR features from TTSs. Visual inspection of these templates do not reveal significant differences between TW Hya and an M0.5 dwarf star, except in the $\mathrm{H}_{2} \mathrm{O}$ band at $1.35 \mu \mathrm{m}$. McClure et al. (2013) also found that all K7-M0 CTTSs appear as M2 dwarf stars in one of their most prominent line ratios, which demonstrates the need to use WTTSs as templates. A composite spectrum of photospheres with different temperatures is not needed to explain the optical spectrum at $<10,000 \AA$, although magnetic spots are expected to affect effective temperature measurements.

\subsubsection{FM Tau}

FM Tau is our most extreme example of a change in spectral type. The most commonly used spectral type of M0 can be traced back to Cohen \& Kuhi (1979). However, the Cohen \& Kuhi (1979) spectra cover 4500-6600 A, where FM Tau looks like an M0 star because of high veiling. Hartigan et al. (1994) twice obtained FM Tau spectra from 5700-7000 A and classified FM Tau as M0 and M2. The prominent TiO absorption bands are readily detected at $>7000 \AA$, where the red photosphere is stronger than the accretion continuum.

FM Tau is classified here as an M4.5 \pm 0.4 . The large uncertainty in spectral type is caused by the high level of veiling. The measured extinction of $A_{V}=0.35 \pm 0.2$ is mostly constrained by fitting to the accretion continuum rather than the photosphere. The systematic uncertainty in $A_{V}$ is caused by the uncertain shape of the accretion continuum. An M0 star is a reasonable approximation for the FM Tau colors, so our extinction is similar to literature values (e.g., Kenyon \& Hartmann 1995; White \& Ghez 2001).

\subsubsection{DG Tau}

DG Tau is the source of a famous and well-studied jet (e.g., Eislöffel \& Mundt 1998; Bacciotti et al. 2000). Literature spectral types range from K3-M0 (Basri \& Batalha 1990; Kenyon \& Hartmann 1995; White \& Hillenbrand 2004), including what should be a reliable spectral type of K3 from high-resolution optical spectra (White \& Hillenbrand 2004). The discrepancies are caused by the high veiling. Gullbring et al. (2000) called DG Tau a continuum star, implying that no photosphere is detectable.

At low resolution, the spectrum shows the 5200 feature, which is typical of $\mathrm{K}$ stars, and $\mathrm{TiO}$ bands, which are seen only in stars with SpT K5 and later (Figure 17). The $\mathrm{SpT}$ is $\mathrm{K} 7_{-1}^{+1}$, with $A_{V}=1.6 \pm 0.15 \mathrm{mag}$. The extinction depends mostly on the shape of the accretion continuum and does not significantly change with a small change in SpT. The SpT is limited to earlier than or equal to M0 by the shallow depth of the $\mathrm{TiO}$ bands.

\subsection{6. $C W T a u$}

CW Tau is a heavily veiled TTS with a jet (e.g., Coffey et al. 2008). Cohen \& Kuhi (1979) classify CW Tau as a K3 star, which is consistent with our measurement. Horne et al. (2012) and Brown et al. (2013) found absorption in CO $v=1-0$ transitions, indicating that our line-of-sight passes through the surface layers of the circumstellar disk. The spectral analysis presented here 
is based on the 2008 January 18 spectrum. The three spectra obtained in 2008 December are eight times fainter because of a change in extinction. This variability will be discussed further in a future paper.

Unlike DG Tau and DL Tau, the CW Tau spectrum does not show any $\mathrm{TiO}$ absorption, which restricts the $\mathrm{SpT}$ to earlier than K5. The presence of the $5200 \AA$ bump suggests that CW Tau is later than K0. Our best fit is a K3 star with $A_{V}=1.74$. The acceptable SpT range is from $\mathrm{K} 1-\mathrm{K} 4$ with corresponding $A_{V}$ of 1.9 and 1.6, respectively. As with other heavily veiled stars, the methodological uncertainty in $A_{V}$ is smaller than would be expected for this range in SpT because of high veiling. However, the systematic uncertainty in extinction may be higher because the extinction relies on the assumption that the accretion continuum is flat.

The observed spectrum is weaker than the model spectrum at $>8000 \AA$, a difference which is also detected in some other accreting stars. The weaker flux indicates a smaller veiling, which could be attributed to the Paschen jump.

\subsubsection{DP Tau}

DP Tau is a 15 AU binary system (Kraus et al. 2011) that appears as a heavily veiled star. The spectral type assigned here of M0.8 is based on the depth of the $\mathrm{TiO}$ bands for accretion continuum veilings of $r_{7510}=0.36,0.38$, and 0.40 for our three spectra. The uncertainty in spectral type is $\sim 0.5$ subclasses and is dominated by the uncertainty in the accretion continuum. The extinction of $A_{V}=0.78 \mathrm{mag}$ is calculated by comparing the observed spectrum to a combined accretion plus photospheric spectrum.

DP Tau is highlighted here as an example of a counterintuitive parameter space, like DM Tau but with higher veiling, where a later spectral type leads to a higher extinction. This behavior for heavily veiled stars is the opposite of expectations for stars without accretion. For DP Tau, if the accretion continuum is increased so that $r_{7510}=0.55$, then the increased depth of the TiO features leads to a SpT of M1.9. However, the combined spectrum of accretion plus photospheric template is bluer than the M0.8+accretion spectrum, so the $A_{V}=0.98 \mathrm{mag}$. Similarly, a low veiling of $r_{7510}=0.28$ leads to M0.6 and $A_{V}=0.56 \mathrm{mag}$. These fits are the limiting cases for reasonable fits to the observed spectrum. The extinction measurements are similar because they are based largely on the shape of the blue accretion continuum, which is assumed to be flat.

\subsection{Comparison of Spectral Types to Previous Measurements}

In this subsection, we compare our spectral types to selected literature measurements. Our internal precision in $\mathrm{SpT}$ is $\sim 0.2$ subclasses for $\mathrm{M}$ dwarfs and $0.5-1$ subclass for earlier spectral types, based on the repeatability of SpT from independent multiple observations of the same stars. In general, the spectral types agree with literature values to $\sim 0.5$ subclasses, as demonstrated in our comparison of spectral types of stars the MBM 12 Association with Luhman (2001). However, significant discrepancies exist for members of the TWA and for some well known members of Taurus. The K5-M0.5 range in SpT may also have systematic offsets of 0.5-1 subclass in SpT relative to other studies.

\subsubsection{Comparison to Luhman Spectral Types}

The spectral type sequence described here is based largely on that established by Luhman. Table 8 compares 29 stars with spectral types and extinctions measured here, in a survey of the
Table 8

Comparison to Luhman Spectral Types

\begin{tabular}{|c|c|c|c|c|}
\hline \multirow[t]{2}{*}{ Star } & \multicolumn{2}{|c|}{ This Work } & \multicolumn{2}{|c|}{ Luhman } \\
\hline & SpT & $A_{V}$ & $\mathrm{SpT}$ & $A_{V}$ \\
\hline MBM 1 & K5.5 & 0.08 & K6 & 0.39 \\
\hline MBM 2 & M0.3 & 1.64 & M0 & 1.17 \\
\hline MBM 3 & M2.8 & 0.54 & M3 & 0.0 \\
\hline MBM 4 & K5.5 & $(-0.24)$ & K5 & 0.85 \\
\hline MBM 5 & $\mathrm{~K} 2$ & 0.88 & K3.5 & 1.95 \\
\hline MBM 6 & M3.8 & 0.50 & M4.5 & 0.0 \\
\hline MBM 7 & M5.6 & $(-0.08)$ & M5.75 & 0.0 \\
\hline MBM 8 & M5.9 & 0.28 & M5.5 & 0.0 \\
\hline MBM 9 & M5.6 & 0.10 & M5.75 & 0.0 \\
\hline MBM 10 & M3.4 & 0.60 & M3.25 & 0.18 \\
\hline MBM 11 & M5.8 & $(-0.08)$ & M5.5 & 0.0 \\
\hline MBM 12 & M2.6 & 0.24 & M3 & 1.77 \\
\hline FU Tau & M6.5 & 1.20 & M7.25 & 1.99 \\
\hline V409 Tau & M0.6 & 1.02 & M1.5 & 4.6 \\
\hline XEST 17-059 & M5.2 & 1.02 & M5.75 & 0.0 \\
\hline XEST 20-066 & M5.2 & $(-0.14)$ & M5.25 & 0.0 \\
\hline XEST 16-045 & M4.5 & $(-0.06)$ & M4.5 & 0.0 \\
\hline XEST 11-078 & M0.7 & 1.54 & M1 & 0.99 \\
\hline XEST 26-062 & M4.0 & 0.84 & M4 & 1.88 \\
\hline XEST 09-042 & K7 & 1.04 & M0 & 0.39 \\
\hline XEST 20-071 & M3.1 & 3.02 & M3.25 & 2.77 \\
\hline $2 \mathrm{M} 0441+2302$ & M4.3 & $(-0.15)$ & M4.5 & 0.39 \\
\hline $2 \mathrm{M} 0415+2818$ & M4.0 & 1.80 & M3.75 & 1.99 \\
\hline $2 \mathrm{M} 0415+2746$ & M5.2 & 0.58 & M5.5 & 0.0 \\
\hline $2 \mathrm{M} \mathrm{0415+2909}$ & M0.6 & 2.78 & M1.25 & 1.99 \\
\hline $2 \mathrm{M} \mathrm{0455+3019}$ & M4.7 & 0.70 & M4.75 & 0.0 \\
\hline $2 \mathrm{M} 0455+3028$ & M5.0 & 0.18 & M4.75 & 0.0 \\
\hline $2 \mathrm{M} \mathrm{0436+2351}$ & M5.1 & -0.18 & M5.25 & 0.34 \\
\hline $2 \mathrm{M} \mathrm{0439+2601}$ & M4.9 & 2.66 & M4.75 & 0.63 \\
\hline
\end{tabular}

MBM 12 Association by Luhman (2001), and in a survey of Spitzer IRAC/X-ray excess sources by Luhman et al. (2009).

The median absolute difference between our and Luhman Mdwarf spectral types is 0.25 subclasses. The standard deviation is 0.37 subclasses. Six objects ( $20 \%$ of the sample) differ by more than 0.5 subclasses. Three of those six objects have spectral types in the K7-M1 range, as expected given the possible differences in our SpT scales (see Section 3.1).

\subsubsection{Taurus Spectral Types}

Many of the most famous objects in Taurus have spectral types that date back to Cohen \& Kuhi (1979), as listed in the compilations of Herbig \& Bell (1988) and Kenyon \& Hartmann (1995). The Cohen \& Kuhi (1979) spectral coverage was optimal for early spectral types but insufficient for $M$ stars. Table 9 lists the most significant changes in Taurus spectral types, relative to the compilation of spectral types by Luhman et al. (2010). Our new spectral types are often two to three subclasses later than those from Cohen \& Kuhi (1979), particularly when veiling affected the spectral typing at short wavelengths. In cases of overlap with D'Orazi et al. (2011), our spectral types are consistent to within 0.5 subclasses of the measured effective temperature.

Several Taurus stars with spectral types earlier than K0 are challenging for spectral type measurements because accretion produces emission in the same lines (e.g., Ca II infrared triplet, $\mathrm{H}$ Balmer lines) that are used for spectral typing. For example, $\mathrm{H} \alpha$ appears in emission from V892 Tau and RY Tau despite early spectral types. While RY Tau has had numerous spectral 
Table 9

Discrepancies in Taurus Spectral Types

\begin{tabular}{lcc}
\hline \hline Star & This Work & Literature $^{\mathrm{a}}$ \\
\hline CIDA 9 & M1.8 & K8 \\
DM Tau & M3.0 & M1 \\
DS Tau & M0.4 & K5 \\
FM Tau & M4.5 & M0 \\
FN Tau & M3.5 & M5 \\
FP Tau & M2.6 & M4 \\
FS Tau & M2.4 & M0 \\
GM Tau & M5.0 & M6.4 \\
GO Tau & M2.3 & M0 \\
IRAS 04216+2603 & M2.8 & M0.5 \\
IRAS 04187+1927 & M2.4 & M0 \\
IS Tau & M2.0 & M0 \\
LkCa 4 & M1.3 & K7 \\
LkCa 3 & M2.4 & M1 \\
RY Tau & G0 & K1 \\
LkHa 332 G1 & M2.5 & M1 \\
LkHa 358 & M0.9 & K8 \\
\hline
\end{tabular}

Notes.

${ }^{a}$ Literature SpT as adopted by Luhman et al. (2010).

${ }^{\mathrm{b}}$ Other recent works have measured early $\mathrm{G}$.

types between F7-G1 (Mora et al. 2001; Calvet et al. 2004; Hernandez et al. 2004), the Cohen \& Kuhi (1979) spectral type of $\mathrm{K} 1$ has been adopted in several recent computations. Our spectral type of G0 for RY Tau agrees with other recent spectral types.

\subsubsection{TWA Association Spectral Types}

Our spectral types for the TWA are uniformly later than the spectral types obtained from Webb et al. (1999; see Table 10). Our spectral types for TWA 8A, TWA 8B, TWA 9A, and TWA 9B are consistent with those obtained from highresolution spectra by White \& Hillenbrand (2004). Our spectral types are also mostly consistent with the recent spectral types measured from X-Shooter spectra by Stelzer et al. (2013), with the exception of TWA 14 (M1.9 here versus M0.5 in Stelzer et al.). The outdated spectral types from Webb et al. (1999) have led to some confusion regarding membership. Weinberger et al. (2013) discuss that space motions may suggest that TWA 9A and $9 \mathrm{~B}$ are not members of the TWA, which they support with ages of 63 and $150 \mathrm{Myr}$, respectively. The later SpT measured here lead to younger age estimates that are consistent with the $\sim 10$ Myr age of the TWA.

In some cases, the age of a star as measured from an H-R diagram may differ from the dynamical or global age of a cluster. For example, with their later spectral type, Vacca \& Sandell (2011) argue that the age of TW Hya is 3 Myr. Our age is now consistent with the global age of the TWA. However, even if the estimated age of a single star were younger, the dynamical age and cluster age are both consistent with 7-10 Myr (e.g., Mamajek 2005; Ducourant et al. 2014). Any deviations from this age for confirmed members are likely due to real scatter in observed photospheric luminosities and temperatures rather than the actual age of the star. These uncertainties are discussed in more detail in Section 5.

\subsection{Comparison of Extinctions to Previous Measurements}

In this subsection, we compare our extinctions to literature extinctions. Our uncertainties are repeatable to $\sim 0.1 \mathrm{mag}$,
Table 10

New TWA Spectral Types

\begin{tabular}{lcc}
\hline \hline Star $^{\mathrm{a}}$ & This Work & Webb et al. 1999 \\
\hline TWA 1 & M0.5 & K7 \\
TWA 2AB & M2.2 & M0.5(+M2) \\
TWA 3A & M4.1 & M3 \\
TWA 3B & M4.0 & M3.5 \\
TWA 4AabBab & K6 & K5 \\
TWA 5AB & M2.7 & M1.5(+M8.5) \\
TWA 6 & M0.0 & K7 \\
TWA 7 & M3.2 & M1 \\
TWA 8A & M2.9 & M2 \\
TWA 8B & M5.2 & M5 \\
TWA 9A & K6 & K5 \\
TWA 9B & M3.5 & M1 \\
\hline
\end{tabular}

Note. ${ }^{\text {a }}$ Unresolved binaries listed as single combined SpT TWA 2 and TWA 5 unresolved here and in Webb et al.

when multiple spectra of the same star are analyzed assuming a constant spectral type. Including uncertainty from spectral type and gravity, our extinctions should be reliable to $\sim 0.2-0.3$ mag Literature uncertainties are commonly claimed to be $\sim 0.2-1.0 \mathrm{mag}$, although statistical errors on the lower end of this range are based on photometric accuracy and are typically not realistic. The primary sources of extinction errors are caused by uncertainty in spectral type, gravity mismatches between the target star and a template for M dwarfs, and the estimates for the shape and strength of the accretion continuum.

Figure 18 shows the comparison of our extinctions with those from several different literature sources. In general, our extinctions agree with literature estimates from optical extinction estimates, but discrepancies with near-IR extinction estimates are large and systematic.

Gullbring et al. $(1998,2000)$ used optical photometry to measure extinctions. The mean difference between our measurements and Gullbring et al. is $0.04 \mathrm{mag}$, with a standard deviation of $0.37 \mathrm{mag}$. Kenyon \& Hartmann (1995) typically use $V-R$ and $V-I$ colors to measure extinctions for the bright stars that dominate overlap between our and their sample, with a difference of $0.1 \mathrm{mag}$ and a standard deviation of $0.7 \mathrm{mag}$ when compared to our results. Given the uncertainties in their and our results, our extinctions are typically consistent with those of Gullbring and KH95. The mean difference and scatter of our extinctions relative to Luhman extinctions are 0.10 and 0.93 mag., respectively.

On the other hand, near-IR analyses yield significantly higher extinctions than those measured at optical wavelengths. The large Furlan et al. (2011) survey of Spitzer IRS spectra included updated extinctions based on fitting photospheric and disk emission to $J H K$ photometry and, in some cases, near-IR spectroscopy. Their extinctions are typically 1.1 mag larger than the $A_{V}$ measured here, with $1.2 \mathrm{mag}$ of scatter after accounting for that bulk shift. Among the sources common to both studies, $5 \%$ of extinctions are different by $>3$ mag, with large discrepancies especially common for strong accretors. These differences are likely caused by the large near-IR excess associated with gas and dust in the inner disk, which can also be characterized by veiling (e.g., Meyer et al. 1997; Folha \& Emerson 1999). Indeed, the J-H extinctions from the White \& Hillenbrand (2004) sample of evolutionarily young stars, which did not account for veiling, are an average of 3.6 mag larger than those measured here. Large differences between optical 

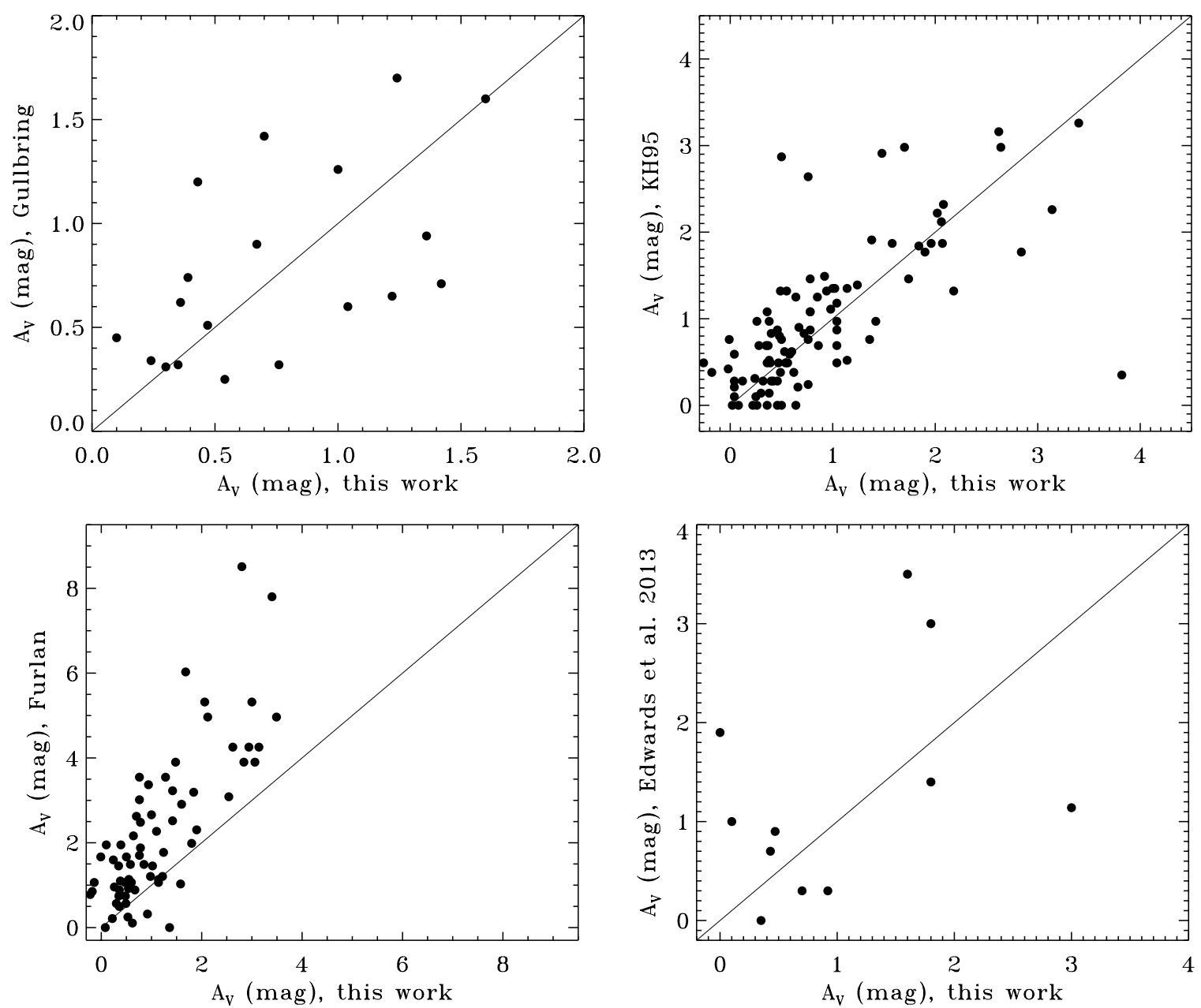

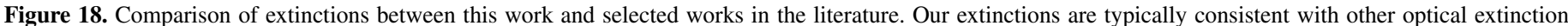

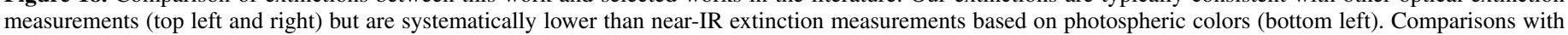

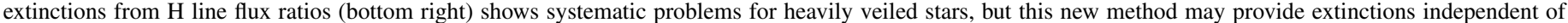
photospheric colors.

and near-IR extinctions were also noted by McJunkin et al. (2014).

Fischer et al. (2011) and McClure et al. (2013) developed a more robust and more painful approach to measure extinctions from flux calibrated near-IR photospheric spectra, after measuring and subtracting the veiling. The veiling in the near-IR is caused by a combination of accretion and warm disk emission. In both studies, the veiling is measured and subtracted off the observed spectrum. Extinction is then measured by comparing the remaining photospheric spectrum to a standard star. However, our extinctions are $1.3 \mathrm{mag}$ lower than those of Fischer et al. (2011), with a scatter of $1.1 \mathrm{mag}$, possibly because of differences in WTTSs templates. ${ }^{9}$ McClure et al. (2013) obtain results closer to ours, with a bulk offset of 0.7 mag and a scatter of $0.7 \mathrm{mag}$. Our extinctions are actually larger than the near-IR extinctions of McClure et al. (2013) in three of the nine stars in their sample. These differences may be related to uncertainties in the near-IR colors of CTTSs and WTTSs, and the lower sensitivity of near-IR spectra to extinction.

\footnotetext{
9 This difference may be attributed to the lack of a sufficient grid of near-IR WTTS templates. One of their two templates, V819 Tau was assigned $A_{V}=2.6$ mag (compared with $1.1 \mathrm{mag}$ here) based on a comparison with a main sequence star. Their other template, LkCa 14, was assigned an M0 spectral type (compared with K5 here). Gullbring et al. (1998) also found anomalous near-IR colors for V819 Tau.
}

Extinctions calculated from line flux ratios could in principle lead to more accurate measurements than photospheric-based extinctions, if the lines are optically thin or other easily modeled and have significantly different wavelengths. Edwards et al. (2013) developed near-IR H Paschen and Brackett line fluxes as an extinction diagnostic. The $\mathrm{H}$ line emission is usually dominated by the accretion flow and should usually have the same line of sight as the stellar photosphere. Our extinctions are 0.27 mag smaller than theirs with a standard deviation of $1.1 \mathrm{mag}$. However, the agreement improves $(0.6 \mathrm{mag})$ when restricted to the four stars in both studies that do not have high veiling and powerful outflows. The heavily veiled outflow sources have larger extinction uncertainties in this work and may have $\mathrm{H}$ line emission with significant outflow contributions.

While we consider optical extinctions more reliable than those in the near-IR, they are inappropriate to use when optical emission from a star is seen primarily scattered light. This criteria applies especially to stars with disks viewed edge-on or stars with remnant envelopes. Some systems like $\mathrm{Sz} 102$ or HL Tau have very low $A_{V}$ measurements but are much fainter than would be expected for a Taurus TTS with their SpT. In these cases, the extinction estimates likely require full spectral energy distribution (SED) modeling and in any case may not be relevant for interpreting the observed optical or near-IR emission from the star. 
The extinction calculations presented here are more accurate than previous measurements for stars in our sample earlier than M5. When veiling is negligible, photometry combined with a reliable spectral type and a template with similar gravity (Pecaut \& Mamajek 2013) may yield a more reliable extinction than flux calibrated spectra. Red or near-IR colors may be preferable to measure extinction to TTSs later than M5 because the optical emission is on the Wien tail of the blackbody distribution and changes quickly as a function of temperature.

\section{OBSERVATIONAL UNCERTAINTIES IN STELLAR PROPERTIES AND CLUSTER LUMINOSITY SPREADS}

Improvements in spectral types and extinctions lead to a more accurate placement on H-R diagrams. Whenever young stellar clusters have been placed on H-R diagrams, a large luminosity spread is measured at a given spectral type (see reviews by Hillenbrand et al. 2008; Preibisch 2012). The observational contribution to luminosity spreads is typically estimated by creating a synthetic cluster of stars with temperatures and luminosities scattered by an amount consistent with the estimated uncertainties. In many cases, the entire spread of luminosities may be explained by observational errors (Hartmann et al. 1998; Slesnick et al. 2006; Preibisch 2012). On the other hand, Reggiani (2011) found that the luminosity spread in HST optical photometry of the Orion Nebular Cluster could not be replicated with purely observational errors. The observational uncertainties in stellar properties, and the uncertainties in the uncertainties, limit our ability to test pre-main-sequence evolutionary tracks, the effect of accretion histories, and the timescale over which star formation occurs within a cluster.

In this section, we describe how the observational uncertainties in spectral type, extinction, and veiling measured in this paper relate to luminosity spreads. Listed uncertainties refer to $\sim 1 \sigma$ error bars, although these measurements are not always rigorous. This description does not include some of the most important uncertainties: multiplicity, partial disk obscuration of the star, cluster membership (see Section 6.2 for a discussion), and stellar spots. In Section 5.5, we present results of improved stellar parameters on the H-R diagrams for the TWA and MBM 12.

\subsection{Direct Luminosity Uncertainties from Distance, Flux Calibration, and Extinction}

The approximate uncertainty in distance is $\sim 10 \%$ to any given star in the Taurus Molecular Cloud, which leads directly to a $20 \%$ uncertainty in luminosity. The depth of the Taurus cloud in our line of sight is likely large compared with the median distance, so the percentage of uncertainty in distance is large. Many of the TWA objects have parallax distances with $<5 \%$ uncertainties. On the other hand, large systematic uncertainties plague the absolute, but not the relative, photometric distance to the MBM12 Association.

The absolute flux calibration, here $\sim 10 \%$, leads directly to the same $10 \%$ uncertainty in luminosity. The relative flux calibration also leads to an uncertainty in the extinction, in this work about 0.1 mag. in $A_{V}$.

Typical extinction uncertainties in $A_{V}$ are $\sim 0.2 \mathrm{mag}$ (or $\sim 0.4$ mag when veiling is significant), which here leads to a $13 \%$ (28\%) luminosity uncertainty from the $7510 \AA$ photospheric flux.

\subsection{Methodological Uncertainties}

These uncertainties are introduced in our approach to fitting spectral type, accretion continuum flux, and extinction simultaneously in each spectrum from a grid of standard WTTSs. The variables are correlated, so changing the spectral type or accretion continuum flux also lead to changes in the extinction.

\subsubsection{Veiling and Stellar Luminosity}

Veiling of the photospheric emission by the accretion continuum and any disk emission increases the observed flux. If the accretion continuum flux is not subtracted, then the measured flux will overestimate the photospheric flux. In this work, the photospheric luminosity is always corrected for veiling and as a result are usually lower than previous estimates. Uncertainties of $\sim 20 \%$ in the strength of the accretion continuum typically lead to $\sim 5 \%$ uncertainties in the final luminosity, with larger uncertainties for higher veiling. This error can be assessed for each target by comparing the veiling to the $7510 \AA$ photospheric flux (see Appendix C and Table 14). Failure to subtract the accretion continuum off from the measured flux will lead to systematically overestimating the stellar luminosity in a way that correlates with veiling and accretion.

\subsubsection{Spectral Type and Luminosity}

We assess internal SpT uncertainties of 0.2 subclasses for $\mathrm{M}$ dwarfs, 0.5 subclasses between K8-M0.5, and 1 subclass for stars between G0 and K8. The spectral types are repeatable to those levels of precision for stars with multiple spectra. These spectral types are optimized with a quantified inclusion of both the accretion continuum flux and reddening and should have smaller uncertainties than spectral types obtained by eyeball comparisons of spectra to a grid of standard spectra. The uncertainty for M-dwarfs relative to literature estimates is $\sim 0.5$ subclasses.

Veiling affects spectral type measurements. Our largest change in spectral type is 4.4 subclasses (for FM Tau), though errors larger than two subclasses would be surprising for the type of red spectra that have been obtained in most studies over the past decade. However, differences of one to two subclasses for veiled spectra can be subtle, as demonstrated for UScoCTIO 33 and DM Tau above.

For an error in spectral type, the bolometric correction and therefore the estimated luminosity of the star does not change significantly, unless the photospheric flux is measured in the Wien tail for the relevant temperature. However, the stellar luminosity at a given age is sensitive to the effective temperature. The comparison between the expected and estimated luminosity thereby introduces a luminosity spread. Figure 19 shows that $3500 \mathrm{~K}$ stars with temperatures overestimated by $300 \mathrm{~K}$ and $75 \mathrm{~K}$ ( $\sim 1.5$ and $0.2 \mathrm{SpT}$, respectively) would appear underluminous by a factor of $\sim 2.8$ and $\sim 1.3$, respectively. This uncertainty may lead to veiled stars, such as UScoCTIO 33, systematically appearing underluminous and old if veiling is not accounted for in the SpT. Some of this underluminosity may be balanced if veiling is also mistakenly unaccounted when converting a band flux to the luminosity.

\subsubsection{Spectral Type and Extinction}

An error in the spectral type introduces a mismatch between the template and object spectra, thereby causing an error in the extinction. Figure 20 shows the uncertainty in $A_{V}$ introduced by the average spectral typing error. In optical 


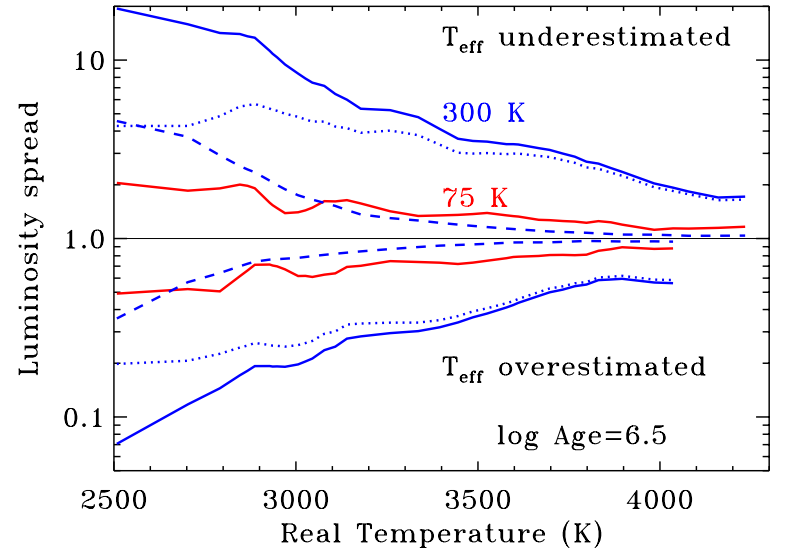

Figure 19. Effect of spectral type and temperature uncertainty on luminosity spread. Stars that have effective temperatures overestimated (or underestimated) will appear underluminous (or overluminous) in an H-R diagram because (a) the expected luminosity at that higher temperature is higher than the expected luminosity at the real temperature, and (b) the bolometric correction is smaller at higher temperatures. The combined curves (solid lines) show the ratio of the measured luminosity and the expected luminosity for a 3.2 Myr old star with temperature overestimated by $75 \mathrm{~K}$ or $\sim 0.5$ subclasses (red) and $300 \mathrm{~K}$ or $\sim 2$ subclasses (blue), based on the Baraffe et al. (2003) pre-main-sequence tracks. The individual components (a, dotted lines) and (b, dashed lines) are shown for the $300 \mathrm{~K}$ error.

(A color version of this figure is available in the online journal.)

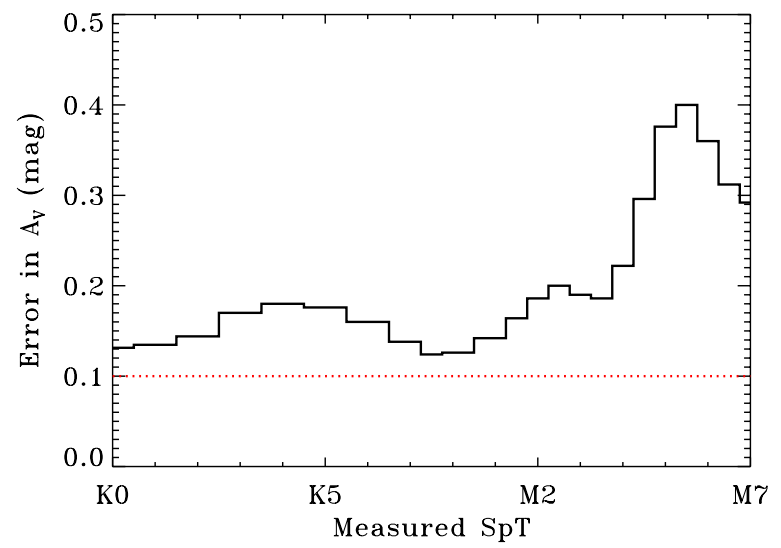

Figure 20. Approximate uncertainty in $A_{V}$ introduced by spectral type uncertainty (solid line) and errors in the relative flux calibration (dotted line). The spectral type uncertainty used here is 1 subclass at spectral types earlier than $\mathrm{K} 7,0.5$ subclasses between $\mathrm{K} 7-\mathrm{M} 1$, and 0.3 subclasses for stars later than M1. An incorrect spectral type will lead to an incorrect extinction because the comparison template has a different photospheric temperature and spectral shape. (A color version of this figure is available in the online journal.)

spectra or photometry, the uncertainty in spectral type dominates extinction uncertainties beyond $\sim$ M5 because the spectral slope changes sharply in the Wien tail. In terms of the luminosity spread, some of the uncertainty introduced by a spectral type error may be partially balanced by the extinction error, which pushes the expected luminosity in the other direction (e.g., da Rio et al. 2010).

\subsubsection{Star Gravity Mismatches and Extinction}

The gravity of the spectral template can also introduce significant errors, even when non-accreting TTSs are used as photospheric templates. Low mass stars with ages of 1-10 Myr have $\log g \sim 3.4-4.2$, depending on the age and mass (see Figure 1). The gravity difference between $\log g \sim 3.4-4.2$

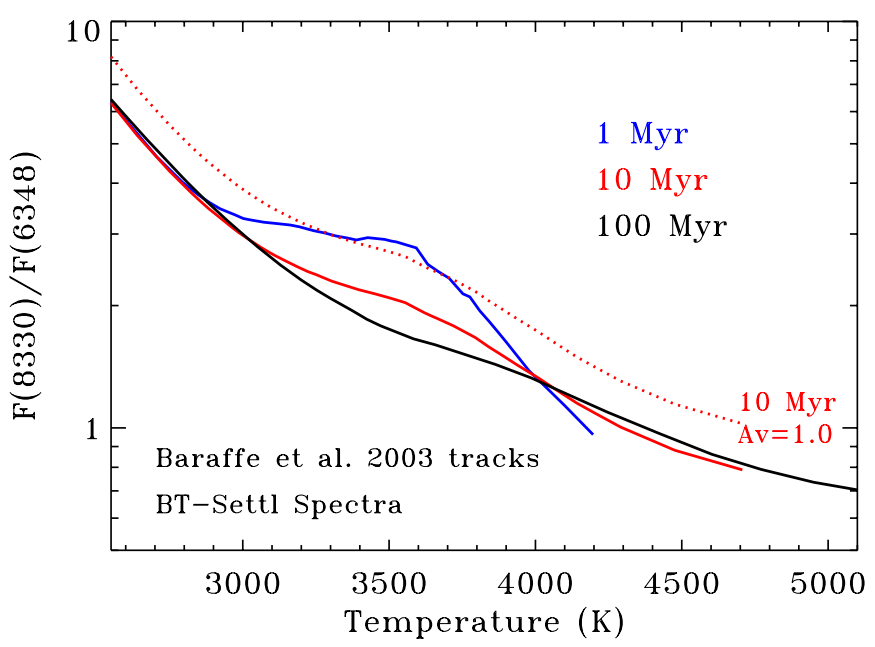

Figure 21. Color dependence, here as $F_{\text {red }}=F(8330) / F(6348)$, for stars with gravity for 1,10 , and $100 \mathrm{Myr}$ old stars. The different colors change the SpT-effective temperature relation (e.g., Forestini 1994) and extinction estimates-even when comparing $1 \mathrm{Myr}$ and $10 \mathrm{Myr}$ old stars. The gravities are obtained from the PMS tracks of Baraffe et al. (1998), and the colors are calculated from the BT-Settl synthetic spectra (Allard et al. 2012).

(A color version of this figure is available in the online journal.)

(1-10 Myr for $0.7 M_{\odot}$ star) leads to maximum errors of $A_{V}=$ $1.1 \mathrm{mag}$ in $F_{\text {red }}=F(8330) / F(6448)$ for M stars (Figure 21).

\subsection{Assumed Standard Relations}

The uncertainties in extinction law and the shape of the accretion continuum are errors that apply systemically to stellar temperature and luminosity measurements. We briefly describe the effects of errors in these assumptions.

\subsubsection{Extinction Law}

The extinction law is assumed to be that of median interstellar grains, with a total-to-selective extinction of $R_{V}=3.1$. Most spectra in our sample can be well fit with $R_{V}=3.1$ and have $A_{V}<3 \mathrm{mag}$, where the mean interstellar extinction law should apply. The differences in the relative flux attenuation between extinction laws is particularly significant at $<5000 \AA$. Grain growth in high extinction regions makes the extinction curve much more gray, with $R_{V}$ as high as 5.5 (Indebetouw et al. 2005). The few stars in our sample that are heavily extincted $\left(A_{V}>5\right)$ are only well fit with $R_{V} \geqslant 4$.

In our optical spectra, for a star with a measured $A_{V}=$ $1.0 \mathrm{mag}$, applying extinction laws with $R_{V}=5$ from Cardelli et al. (1989) or $R_{V}=5.1$ from Weingartner \& Draine (2001) would lead to $A_{V}=1.2$ and $1.15 \mathrm{mag}$, respectively. The difference in luminosity is minimal for low extinctions. However, an extinction $A_{V}=10$ mag and $R_{V}=5$ will be measured (in red-optical spectra or colors) as $A_{V}=8.3 \mathrm{mag}$ if $R_{V}=3.1$ is assumed, yielding a factor of 5.6 difference in luminosity if assessed at $7510 \AA$.

\subsubsection{Uncertain Shape of Accretion Continuum}

Our analysis relies on an assumption that the accretion continuum flux is constant in erg cm $\mathrm{cm}^{-2} \mathrm{~s}^{-1} \AA^{-1}$ versus wavelength. While this assumption is reasonable, it may not apply to some sources (see Figure 10). A negative slope (stronger emission at $4000 \AA$ than $8000 \AA$ ) would lead to the inference of an earlier spectral types because the veiling would be weaker, so the photospheric $\mathrm{TiO}$ features would not be as deep. For sources with 
Table 11

SpT, $A_{V}$, and the Accretion Continuum Slope

\begin{tabular}{|c|c|c|c|c|c|c|}
\hline \multirow[t]{2}{*}{ Star $^{\mathrm{a}}$} & \multicolumn{2}{|c|}{ Red Slope } & \multicolumn{2}{|c|}{ Flat Slope ${ }^{b}$} & \multicolumn{2}{|c|}{ Blue Slope } \\
\hline & SpT & $A_{V}$ & SpT & $A_{V}$ & SpT & $A_{V}$ \\
\hline UScoCTIO 33 & M4.6 & 0.06 & M4.4 & 0.38 & M4.3 & 0.52 \\
\hline DF Tau & M3.4 & 0.20 & M2.7 & 0.18 & M2.5 & 0.18 \\
\hline DM Tau & M3.4 & 0.12 & M3.0 & 0.08 & M2.8 & 0.08 \\
\hline DP Tau & M1.7 & 0.60 & M1.0 & 0.68 & M0.3 & 0.90 \\
\hline DR Tau & (K6) & 0.56 & (K6) & 0.50 & (K6) & 0.46 \\
\hline GM Aur & K8.5 & 0.14 & K6.5 & 0.36 & K6.5 & 0.40 \\
\hline TW Hya & M1.1 & 0.0 & M0.9 & 0.08 & M0.7 & 0.12 \\
\hline $\mathrm{ZZ} \mathrm{Tau}{ }^{\mathrm{d}}$ & M4.4 & 0.54 & M4.4 & 0.56 & M4.3 & 0.58 \\
\hline
\end{tabular}

Notes. $F(2 \lambda)=2 F(\lambda)$ and $0.5 F(\lambda)$ for red and blue slopes.

a All observations except UScoCTIO 33 from 2008 December 29.

${ }^{\mathrm{b}}$ Results may differ slightly from best fits using all dates.

${ }^{\mathrm{c}}$ High veiling, so K6 SpT assumed for DR Tau.

${ }^{\mathrm{d}}$ Example of little change because of weak veiling.

moderate or strong veiling, the extinction would be underestimated in our paper.

DM Tau is used here as an example of the effect of the shape of the veiling continuum for a moderately veiled star. If the accretion continuum is two times weaker at $8000 \AA$ than at $4000 \AA$, then the best-fit model is M2.7 with $A_{V}=-0.02$ mag. If instead the accretion continuum is two times stronger at $8000 \AA$ than at $4000 \AA$, then the best-fit model is M3.6 and $A_{V}=$ $0.2 \mathrm{mag}$. The different spectral types are caused by different $\mathrm{TiO}$ absorption depths. The extinction does not change significantly because the color change in the accretion continuum is offset by a color change in spectral type.

Table 11 describes a similar analysis for a few stars with a range of veilings. The synthetic spectra with an accretion continuum that get brighter to longer wavelengths are typically bad fits to the observed spectra. For heavily veiled stars, the change in extinction could be as large as $A_{V}=0.9 \mathrm{mag}$. Spectral types of heavily veiled stars around $\mathrm{K} 7$ are especially dependent on the shape of the veiling continuum. The real uncertainty in SpT and $A_{V}$ are likely smaller than the differences described in this paragraph because the accretion continuum is likely much closer to a flat spectrum at optical wavelengths.

\subsubsection{Conversion from Spectral Type to Temperature}

The temperature scale for pre-main-sequence likely has an uncertainty of $\sim 50 \mathrm{~K}$ for early $\mathrm{M}$ dwarfs and $\sim 100 \mathrm{~K}$ for late $\mathrm{M}$ dwarfs based on the comparisons between different temperature scales described in Section 3.2. In addition to these uncertainties, the models themselves have some uncertainty. Any error in the temperature scale will apply systematically throughout the entire sample and does not introduce a luminosity spread for stars with similar spectral types. The conversion from spectral type to temperature applied here is measured from our spectral type scale.

\subsubsection{Bolometric Corrections}

Our bolometric corrections are calculated from the BT-Settl model spectra (Allard et al. 2012). The mismatch between models and real spectra may introduce small systematic errors into our luminosity calculations. As with the conversion from spectral type to temperature, this error should not introduce a significant luminosity spread for stars with similar spectral types.

\subsection{Stellar Properties of Heavily Veiled Stars}

Some stars are so heavily veiled that the veil is similar to a burqa, almost completely hiding the photosphere. These stars pose particularly difficult problems for H-R diagrams. In this section, we highlight two problems that may preferentially affect measuring photospheric emission.

\subsubsection{Photospheric Emission of Heavily Veiled Stars}

The age of a pre-main-sequence star is calculated from the contraction timescale and the effective temperature. In most comparisons between data and model spectra, the photospheric luminosity is used as a proxy for the radius. This surface area does not include the fraction of the star covered by the spot. In the shock models of Gullbring et al. (1998), corrections are less than $\sim 20 \%$ and are not significant. However, the shock models of Ingleby et al. (2013) include components at lower density than Gullbring et al. in order to explain veiling at red wavelengths, in excess of previous models. In three cases-RW Aur A, DR Tau, and CV Cha-the accretion shock covers $20 \%-40 \%$ of the stellar surface. In extreme cases, especially for outbursts or Class I objects, some estimate of this covering fraction would need to be combined with the photospheric surface area to calculate a stellar radius. This uncertainty is ignored in this work.

\subsubsection{TiO in Emission and Spectral Types}

Hillenbrand et al. (2012) found $\mathrm{TiO}$ in emission from two Class I sources and one CTTS undergoing an outburst (see also Covey et al. 2011). In our sample, VV CrA and the 2008 January $\mathrm{GV} \mathrm{Tau}^{10}$ spectrum show TiO in emission (Figure 22). Emission lines blanket the optical spectra of VV CrA, GV Tau, and three objects described by Hillenbrand et al. (2012). All objects with $\mathrm{TiO}$ emission have evidence from their SEDs that an envelope is present.

The $\mathrm{TiO}$ emission must be related in some way to strong accretion. The warm $\mathrm{TiO}$ gas is likely located in a warm disk surface, which may be viscously heated by the accretion flow. $\mathrm{TiO}$ emission has only been detected in clear cases. Presumably other CTTSs have weak $\mathrm{TiO}$ emission that would require a dedicated search to detect. The possible complications of $\mathrm{TiO}$ emission filling in absorption bands has not been considered here or in other work but would severely complicate spectral typing. Most likely, these complications arise for only Class I stars.

\subsection{Luminosity Spreads of Loose Associations}

Young stars start to grow out of adolescence when the velocity dispersion of their parent cluster leads them to venture far from their birthplace. At this stage, they are in loose associations with their siblings and are typically free of extinction. In this subsection, we apply the previous description of luminosity spreads to stars in two nearby associations, the TWA and the MBM 12 Association. Both associations are relatively small, with 10-30 known members. The luminosity spread of Taurus is not discussed here because our sample is incomplete and biased and because Taurus includes many subclusters with large age differences.

\footnotetext{
10 The 2008 December observation of GV Tau does not show obvious TiO emission, although this emission may be masked by additional red continuum emission. The variability may be real or attributable to different slit positions and seeing. GV Tau is the one source in our sample that is clearly extended in emission lines beyond what would be expected for a $1^{\prime \prime} .2$ binary, even in poor seeing.
} 

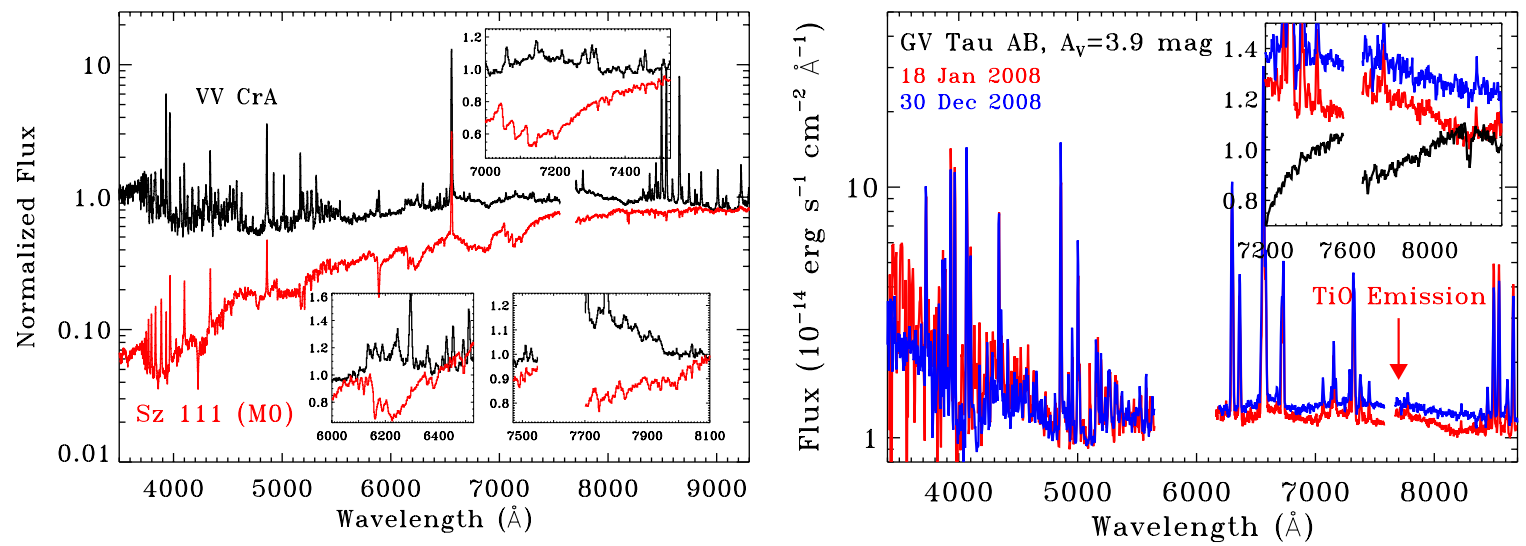

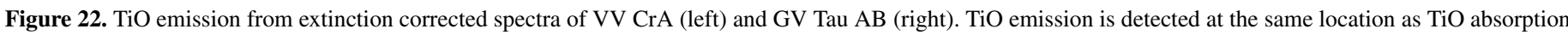

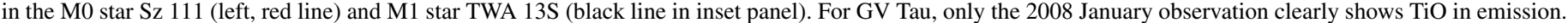
The blue emission lines are also stronger in the 2008 January observation than in 2008 December.

(A color version of this figure is available in the online journal.)
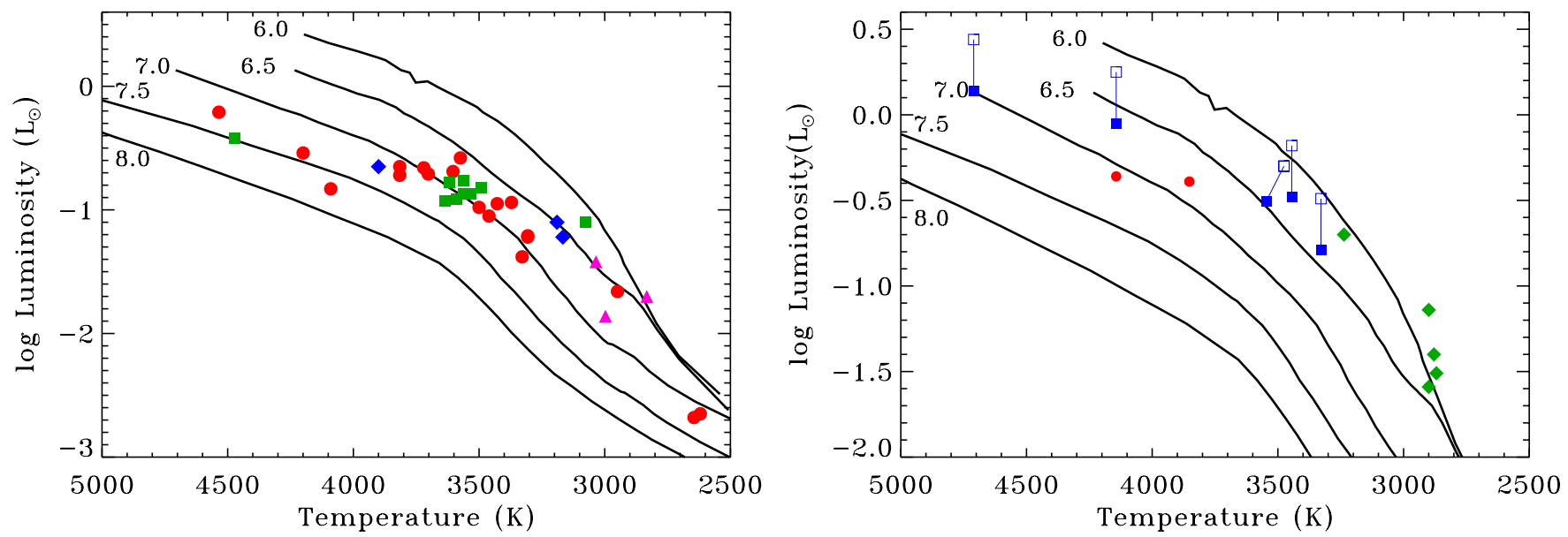

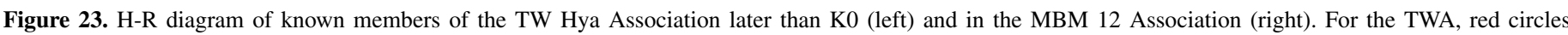

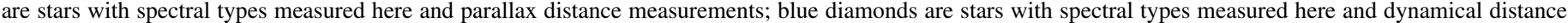

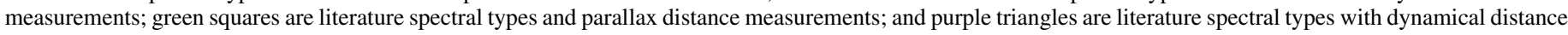

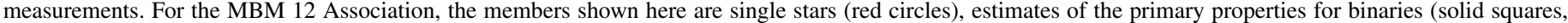

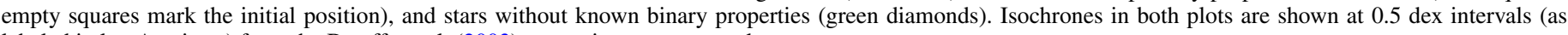
labeled in log Age in yr) from the Baraffe et al. (2003) pre-main-sequence tracks.

(A color version of this figure is available in the online journal.)

\subsubsection{Luminosity Spread of the TW Hya Association}

The nearby TWA is a loose association of $\sim 30$ stars with an age of $\sim 10 \mathrm{Myr}$ (Webb et al. 1999). The members are especially meaningful for age estimates because of proximity, prevalence of parallax measurements, negligible extinction, and near-complete accounting of binarity.

Since the association is not near any molecular cloud, extinction is assumed to be zero for most members. Two stars, TWA 30A and 30B, have disks that are nearly edge-on and may attenuate photospheric emission (Looper et al. 2010a, 2010b) and are therefore ignored in this analysis. Although Ducourant et al. (2014) calculate and apply extinction corrections to several TWA members, the color corrections may be introduced by small errors in spectral type and at present are not accurate enough to use anything other than $A_{V}=0$ mag for members of this association.

Figure 23 shows the H-R diagram and luminosity spread of the TWA. Table 12 lists the stellar parameters of TWA members, including an age from the Baraffe et al. (2003) premain-sequence evolutionary tracks and a ratio $L / L 10$ of the measured luminosity to $L 10$, the 10 Myr luminosity for the relevant temperature. Most of the luminosities are calculated from our optical spectra. The luminosities of several close binaries are obtained from HST narrowband imaging at $1.64 \mu \mathrm{m}$ Weintraub et al. (2000), while others are obtained from the $J$-band magnitude measured in 2MASS. All total fluxes are calculated using bolometric corrections calculated from the BT-Settl spectra. The binary systems TWA 3Aab, TWA 5Aab, TWA 16AB, TWA $23 \mathrm{AB}$, and TWA $32 \mathrm{AB}$ are roughly equal mass (Muzerolle et al. 2000; Zuckerman et al. 2001; Shkolnik et al. 2011; Weinberger et al. 2013), so the luminosities used for this analysis are divided by 2. For HD $98800 \mathrm{Aa}, \mathrm{Ba}$, and Bb, we used the temperatures calculated by Laskar et al. (2009). The luminosities of HD $98800 \mathrm{Bab}$ are calculated from the absolute $K$-band magnitudes of Boden et al. (2005), adjusted for distance. The luminosity of HD $98800 \mathrm{Aa}$ is calculated from the $K$-band flux in Prato et al. (2001).

The combined TWA $2 \mathrm{AB}$ spectrum is classified here as an M2.2 object. The color difference suggests a 1.5-2 subclass difference between the pair. To determine the spectral type of the primary, we added scaled template spectra together and 
Table 12

Stellar Ages in the TWA ${ }^{\mathrm{a}}$

\begin{tabular}{|c|c|c|c|c|c|}
\hline Star & $\begin{array}{c}d \\
(\mathrm{pc})\end{array}$ & $\mathrm{SpT}$ & $\log L / L_{\odot}$ & $\log$ Age & $L / L 10$ \\
\hline \multicolumn{6}{|c|}{ SpT and Flux from this work } \\
\hline TW Hya & 54 & M0.5 & -0.72 & 7.21 & 0.70 \\
\hline TWA 2A & 42 & $\sim \mathrm{M} 1.7^{\mathrm{b}}$ & $-0.69^{c}$ & 6.79 & 1.39 \\
\hline TWA 2B & 42 & $\sim \mathrm{M} 3.5^{\mathrm{b}}$ & $-1.22^{\mathrm{c}}$ & 6.86 & 1.31 \\
\hline TWA 3Aab & $(35)$ & M4.1 & -1.22 & 6.57 & 2.83 \\
\hline TWA 3B & $(35)$ & M4.0 & -1.10 & 6.49 & 3.34 \\
\hline TWA 5Aab & 49 & M2.7 & -1.05 & 6.80 & 1.36 \\
\hline TWA 6 & (67) & M0 & -0.65 & 7.25 & 0.67 \\
\hline TWA 7 & 34 & M3.2 & -0.94 & 6.64 & 1.80 \\
\hline TWA 8A & 43 & M2.9 & -0.95 & 6.80 & 1.36 \\
\hline TWA 8B & 39 & M5.2 & -1.66 & 6.55 & 2.76 \\
\hline TWA 9A & 47 & K6 & -0.83 & 7.71 & 0.30 \\
\hline TWA 9B & 52 & M3.4 & -1.38 & 7.11 & 0.81 \\
\hline TWA $13 \mathrm{~N}$ & 56 & M1.1 & -0.71 & 7.02 & 0.97 \\
\hline TWA 13S & 59 & M1.0 & -0.66 & 6.98 & 1.03 \\
\hline HR 4796A & 73 & A0 & 1.20 & $\ldots$ & $\ldots$ \\
\hline TWA 14 & 96 & M1.9 & -0.58 & 6.61 & 1.96 \\
\hline TWA 23 AB & 49 & M3.5 & -1.21 & 6.84 & 1.37 \\
\hline TWA 25 & 54 & M0.5 & -0.65 & 7.12 & 0.83 \\
\hline TWA 27 & 52 & M8.25 & -2.68 & 7.18 & 0.73 \\
\hline TWA 28 & 55 & M8.5 & -2.65 & 7.09 & 0.84 \\
\hline \multicolumn{6}{|c|}{ SpT and Flux from literature } \\
\hline TWA 5B & (49) & M9 & $-2.83^{b}$ & 7.15 & 0.76 \\
\hline TWA 10 & 62 & M2 & -0.87 & 6.97 & 1.06 \\
\hline TWA 11B & (67) & $\mathrm{M} 2.5$ & $-0.82^{\mathrm{a}}$ & 6.75 & 1.51 \\
\hline TWA $11 \mathrm{C}$ & 69 & M4.5 & $-1.10^{\mathrm{a}}$ & 6.28 & 5.99 \\
\hline TWA 12 & 64 & M1.6 & $-0.78^{\mathrm{a}}$ & 6.96 & 1.07 \\
\hline TWA $15 A$ & $110^{\mathrm{c}}$ & M1.5 & $-0.93^{\mathrm{a}}$ & 7.21 & 0.71 \\
\hline TWA 15B & $117^{\mathrm{c}}$ & $\mathrm{M} 2.2$ & $-0.87^{\mathrm{a}}$ & 6.90 & 1.18 \\
\hline TWA $16 A B$ & 78 & M1.8 & $-0.91^{\mathrm{a}}$ & 7.09 & 0.87 \\
\hline TWA 20 & 77 & $\mathrm{M} 2.0$ & -0.76 & $6.82^{\mathrm{a}}$ & 1.35 \\
\hline TWA 21 & 51 & K3.5 & -0.42 & $7.48^{\mathrm{a}}$ & 0.42 \\
\hline TWA 26 & 38 & M9.0 & -2.85 & 7.22 & 0.74 \\
\hline TWA 29 & 79 & M9.5 & -2.95 & 7.27 & 0.70 \\
\hline TWA 30A & $(56)$ & M5 & \multicolumn{2}{|c|}{ Edge-on disk } & \\
\hline TWA 30B & $(56)$ & M4 & \multicolumn{2}{|c|}{ Edge-on disk } & \\
\hline TWA 32 AB & $(77)$ & M6.3 & -1.70 & $<6$ & 3.88 \\
\hline TWA 33 & $(52.6)$ & M4.7 & -1.42 & 6.48 & 3.56 \\
\hline TWA 34 & $(50)$ & M4.9 & -1.86 & 6.77 & 1.59 \\
\hline HD $98800 \mathrm{Aa}$ & 45 & 4535 & -0.21 & 7.28 & 0.60 \\
\hline HD $98800 \mathrm{Ba}$ & 45 & 4200 & -0.54 & 7.40 & 0.51 \\
\hline HD $98800 \mathrm{Bb}$ & 45 & 3500 & -0.98 & 6.91 & 1.16 \\
\hline
\end{tabular}

Notes. Restricted to TWA members with distances Parallaxes from Weinberger et al. (2013), Malo et al. (2013), Teixeira et al. (2008), Gizis et al. (2007), Biller \& Close (2007), van Leeuwen (2007), and Ducourant et al. (2014). Dynamical distances in "()" (Mamajek 2005). Literature SpT from Konopacky et al. (2007), Webb et al. (1999), Kastner et al. (2008), Shkolnik et al. (2011), Gizis (2002), Zuckerman et al. (2001), Zuckerman \& Song (2004), Looper et al. (2010a, 2010b), Bonnefoy et al. (2009), Schneider et al. (2012), and Allers \& Liu (2013).

${ }^{\text {a }} L$ from $J$-band magnitude (Skrutskie et al. 2006).

${ }^{\text {b }} L$ from $H$-band magnitude (Weintraub et al. 2000).

${ }^{c}$ Large distance may indicate non-membership.

subsequently calculated a new spectral type and extinction. As the secondary-to-primary mass ratio decreases, the secondary reduces the inferred temperature of the total system, thereby also decreasing the expected luminosity. With complete optical wavelength coverage, the resulting spectral type is typically 0.5 subclasses later than the primary SpT and the extinction is within $0.1 \mathrm{mag}$. TWA 2A is assigned a spectral type of M1.7 and used in the analysis below. TWA $2 \mathrm{~B}$ is tentatively assigned a spectral type of M3.5 and is not used in the analysis.

Between K5-M3.5, the average scatter in luminosity is 0.39 dex relative to a $9 \mathrm{Myr}$ isochrone. This scatter is dominated by TWA 9A and 9B and TWA 14 (see Table 12). Weinberger et al. (2013) suggest that TWA 9A and 9B are too old to be TWA cluster members. Ducourant et al. (2014) also note that the kinematic distance for TWA 9A is highly discrepant with the parallax distance. However, Malo et al. (2013) assign a dynamical membership probability of $99 \%$. $\mathrm{H} \alpha$ emission, $\mathrm{Li}$ absorption strength, and gravity indicators show that both stars are young. Our ages of TWA 9A and 9B are younger than the Weinberger et al. (2013) estimate because of later spectral types measured here, though the pair is still underluminous. Between M4-M7, most stars become significantly overluminous for a $10 \mathrm{Myr}$ age, according to these isochrones. Some of this overluminosity could be reduced if the objects are hotter than the SpT-effective temperature conversion (Section 3.2). The brown dwarfs at $\sim \mathrm{M} 8$ are near the predicted $\mathrm{H}-\mathrm{R}$ diagram location for the $10 \mathrm{Myr}$ isochrone.

A more empirical approach suggests that the slope of luminosity versus temperature is much flatter between K0-M5 than the isochrone. A best fit line to the K0-M5 objects yields a luminosity spread of only $0.13 \mathrm{dex}$ and is able to recover the objects down to $3000 \mathrm{~K}$ (compared with $3300 \mathrm{~K}$ for the $10 \mathrm{Myr}$ isochrone). Only one object, TWA 9B, is severely underluminous relative to the line. Such a fit would suggest that the contraction timescale for very low mass stars is longer than predicted, relative to the contraction timescales of solar mass stars and brown dwarfs. Empirical isochrones crossing theoretical isochrones in this manner is not uncommon (e.g., Hillenbrand et al. 2008).

Of the resolved stars in multiple systems, the four closest in spectral type are co-eval to within a luminosity of $20 \%$. The exceptions, such as TWA 8A/8B and TWA 5Aab/5B, have large differences in spectral type, often with one in the problematic M4-M6 spectral type range. The discrepancy points to an error in either effective temperature measurements or in pre-mainsequence tracks rather than a real luminosity spread.

\subsubsection{Luminosity Spread of the MBM 12 Association}

The MBM 12 cloud was initially found by Magnani et al. (1985). A census of MBM 12 revealed a total of 12 stellar systems (Luhman 2001). The approximate distance of 275 pc was calculated by comparing the luminosities to other nearby star forming regions. The age $(\sim 1-5)$ Myr and the distance are degenerate parameters. Seven of the 12 objects retain a disk (Meeus et al. 2009), which is consistent with a $<5$ Myr age.

A binary census of the eight brightest targets revealed seven multiple star systems (Chauvin et al. 2002; Brandeker et al. 2003). Based on near-IR photometry of these multiple systems, four (MBM12 1, 3, 5, and 10) are roughly equal mass stars, so their measured fluxes here are divided by two to estimate the luminosity of a single star. MBM12 12 (S018) is a triple system where the primary is a single star while the secondary is a resolved binary. Based on the near-IR colors, we divide the optical flux by a factor of 1.6 and shift the primary spectral type to M2.1 (from M2.6). Two other binaries, MBM12 4 $(\mathrm{LkH} \alpha 264)$ and MBM12 $2(\mathrm{LkH} \alpha$ 262) are widely separated are not affected by possible companions.

The resulting H-R diagram (Figure 23) is roughly consistent with a 3 Myr age, if the 275 pc distance is accurate. As with the TWA, stars hotter than $4000 \mathrm{~K}$ are fainter than expected 
from the $3 \mathrm{Myr}$ isochrone. Brown dwarfs cooler than $3000 \mathrm{~K}$ are brighter than the $3 \mathrm{Myr}$ isochrone and may be affected by unaccounted binarity. For stars warmer than $3000 \mathrm{~K}$, the luminosity spread about a best fit line is $26 \%$. The slope of the line is $5.8 \times 10^{-4} \log L \mathrm{~K}^{-1}$, almost exactly the same as the slope of $6.6 \times 10^{-4} \log L \mathrm{~K}^{-1}$ for the TWA. The most underluminous star, MBM $2(\mathrm{LkH} \alpha 262)$, is an accretor with an anomalously large extinction $\left(A_{V}=1.75\right)$ relative to other stars in the association. Some obscuration by the central star or a gray extinction law could lead to this underluminosity.

\section{DISCUSSION}

\subsection{The Limited Affect of Accretion Histories on Pre-main-sequence Evolution}

The pre-main-sequence models of Baraffe \& Chabrier (2010) demonstrate that stars contract faster if they form primarily by large accretion events with rates of $>10^{-4} M_{\odot} \mathrm{yr}^{-1}$, as opposed to steady accretion throughout the protostellar lifetime. If the large accretion events are episodic and randomly distributed in strength, then this evolution predicts a significant luminosity spread in observed H-R diagrams (Baraffe et al. 2012). The evolutionary effects will remain large at $10 \mathrm{Myr}$ for $0.1 M_{\odot}$ stars, with luminosity differences up to a factor of 25 , but may be minimal for solar mass stars, depending on the seed mass of the star and size of episodic accretion bursts.

The stars in the TWA are roughly coeval, as are those in MBM 12. Stars in multiple star systems in the TWA also tend to be co-eval with each other. Similarly, in Taurus, two-thirds of binaries have ages consistent to 0.16 dex, with many of the outliers attributable to veiling or other observational uncertainties (Kraus \& Hillenbrand 2009; see also Hartigan et al. 1994; Hartigan \& Kenyon 2003; White \& Ghez 2001).

If episodic accretion dominates stellar growth to an extent that the evolutionary tracks are severely altered, these results would require that the effects of episodic accretion are similar for the majority of stars. In this case, the ages of pre-main-sequence stars would be significantly and uniformly overestimated in all regions. However, the $\sim 7-10 \mathrm{Myr}$ age of the TWA obtained from comparison to pre-main-sequence tracks is similar to the dynamical expansion age of $7.5 \pm 0.7 \mathrm{Myr}$ (Ducourant et al. 2014).

These comparisons suggest that large episodic accretion outbursts do not significantly alter pre-main-sequence evolution over general populations of stars with masses $0.3-0.7 M_{\odot}$. Any affects on entire populations are minimal by ages of 5-10 Myr. However, such events may alter the evolutionary course of a minority of stars and could, in principle, explain the underluminosity of a star like TWA 9A.

\subsection{Minimizing Observational Errors in H-R Diagrams}

The uncertainties in effective temperature and luminosity explains at least some of the luminosity spreads measured within young clusters. In addition to the uncertainties described in Section 5, membership, binarity, and disk obscuration can severely affect measured luminosity spreads in H-R diagrams.

The choice of star forming region and observed wavelength largely determines which uncertainties are minimized and which are problematic. At present, the spectroscopic and direct imaging binary census in Taurus is relatively complete, at least for the well known solar mass members (White \& Ghez 2001; Kraus $\&$ Hillenbrand 2009; Nguyen et al. 2012). In ideal cases where the two stars are diskless and have the same extinction, an accounting of multiplicity to 0.2 times the mass of the primary star yields a $\sim 0.1$ dex error in age. Binarity is a much more severe problem for more distant regions and likely requires the use of population synthesis models to interpret luminosity spreads. Use of near-IR colors to calculate mitigates the effect of extinction uncertainties; however, systematic extinction offsets may be prevalent. The relative distance uncertainty in nearby regions is currently much larger than that for more distant regions.

The near-IR has some advantages over optical spectra. Veiling corrections are less important for red or near-IR flux measurements of late $\mathrm{M}$ dwarfs. The emission produced by accretion and warm dust reaches a minimum between 1-1.5 $\mu \mathrm{m}$, while the photospheric flux from late M dwarfs peak at those same wavelengths. For these late M dwarfs, red or near-IR colors should be used for extinction estimates because they are relatively constant with spectral type (e.g., Leggett 1992) and usually include negligible contribution from veiling. However, extinction estimates are less sensitive and less reliable when measured from near-IR observations.

Probing ages of young ( $<5 \mathrm{Myr}$ ) clusters requires an assessment of the effects of disk parameters on the measured stellar properties. Partial disk obscuration of the star significantly decreases measured luminosities and is particularly difficult to account for. Within our biased sample, $\sim 10 \%$ of accreting objects are significantly underluminous relative to the expected luminosity of a pre-main-sequence star. Some of these faint objects have disks viewed edge-on, which blocks the light from the star. In cases such as AA Tau (Bouvier et al. 2013) and perhaps CW Tau, a disk warp periodically or stochastically blocks the light from the central star (see also Alencar et al. 2010; Findeisen et al. 2013). The measured luminosities of these objects are not realistic and should be discarded from population studies of ages obtained from H-R diagrams. The difficulty is in knowing which stars to discard. Easy cases will appear below the zero age main sequence on an H-R diagram, but many cases will not be so obvious. The inclusion of this uncertainty will require measurements of the frequency and scale of such events from monitoring observations, such as those done by CoRoT (Alencar et al. 2010). When variability information is available, the most straightforward technique is to simply calculate the average stellar brightness. However, in the case of disk obscuration, the maximum photospheric luminosity is likely more appropriate.

\section{PROSPECTS FOR FUTURE IMPROVEMENTS AND CONCLUSIONS}

This paper provides a consistent and robust set of spectral types and extinctions for 281 young stars, including many of the most well studied. The primary advances in this paper are the implementation of simultaneous measurements of the extinction, accretion continuum flux, and spectral type for accreting stars and a sufficient sample size to obtain a robust set of extinction-corrected spectral templates. The effects of veiling on spectral type and extinction are reduced when analyzing spectra with coverage from 4000-9000 A. A similar approach was recently used by Manara et al. (2013a) to investigate two stars with previously reported ages of $30 \mathrm{Myr}$. Their accurate spectral type and luminosity yielded an age of 2-3 Myr, consistent with the age of the parent cluster.

An updated grid of photospheric M star templates will eventually be needed to account for the evolution of colors with pre-main-sequence contraction. Unfortunately, this problem is 
challenging to solve because the TTSs with known $A_{V}=0$ mag. are those in the 7-10 Myr old TWA and the $\eta$ Cham association. No TTS in a young ( $<3 \mathrm{Myr}$ ) region can be assumed to have $A_{V}=0 \mathrm{mag}$ (or any other $A_{V}$ ) based only on its colors, independent of a model template. The high binary fraction of young WTTSs (Kraus et al. 2012) also affects their use as photospheric templates. Although we minimize the effects of gravity dependence by using TTSs as templates, the gravity dependence between 1-10 Myr may still be significant and is not accounted for. Photometric samples of non-accretors are likely reliable, but degeneracy between spectral type and accretion continuum flux can lead to spectral type uncertainties of at least two subclasses.

The approach to measuring spectral types and extinction in this paper can reach a luminosity accuracy of $\sim 0.1-0.2$ dex for most CTTSs, and should serve as a particularly useful guide in the analysis of broadband spectra obtained by VLT/X-Shooter (e.g., Manara et al. 2013a, 2013b) and for analysis of Gaia observations. The grid of spectral types should be improved and based on more direct measurements of effective temperature by comparing high-resolution spectra to models. The spectral typeeffective temperature conversions are also uncertain at present because model atmospheres fail to reproduce some large spectral features for spectral types later than M4. The relationship between spectral type and extinction needs particular improvement between K5-M0.5, where the accuracy of our grid relative to other publications is especially uncertain. Our results rely upon the assumption that the accretion continuum flux is flat. However, the strength of the broadband accretion continuum should be measured with simultaneous broadband spectra. Finally, extinction measurements should include the effect of gravity on photospheric emission, following the gravity-dependent colors obtained by Covey et al. (2007) and Pecaut \& Mamajek (2013). Ideally, some optically thin line ratios could be found and used to measure extinction, independent of gravity.

We thank the referee for helpful comments that improved the clarity and robustness of the results. We appreciate valuable discussions with Suzan Edwards, Adam Kraus, Sylvie Cabrit, Kevin Covey, and Davide Fedele, and also thank Kraus for help with a Taurus membership database. G.J.H. appreciates financial support for this project provided by the Youth Qianren Program of the National Science Foundation of China and the Observatoire de Paris for hosting him as a visiting astronomer.

\section{APPENDIX A}

Our observations include two possible members of Taurus, a background supergiant that was a candidate member of Lupus, and a reflection nebulosity. These tangential results are discussed below.

\section{A.1. GK Tau B}

GK Tau B is a visual companion located 2".4 from GK Tau (Hartigan et al. 1994). Based on optical photometry, White \& Ghez (2001) suggested that the star may be a visual binary that is not associated with the Taurus star forming region.

Our 2008 January 20 observations of GK Tau A and GK Tau B were obtained with slits placed perpendicular to the position angle of the two stars. Some bleeding from GK Tau A likely affects the GK Tau B spectrum. The MgH band, Ca II IRT absorption, and $\mathrm{H} \alpha$ emission are all detected from GK Tau $\mathrm{B}$ at a level that is inconsistent with possible bleeding from GK Tau A. GK Tau B is consistent with $\mathrm{a} \sim \mathrm{K} 3 \mathrm{SpT}$ and $A_{V}=2.1$.

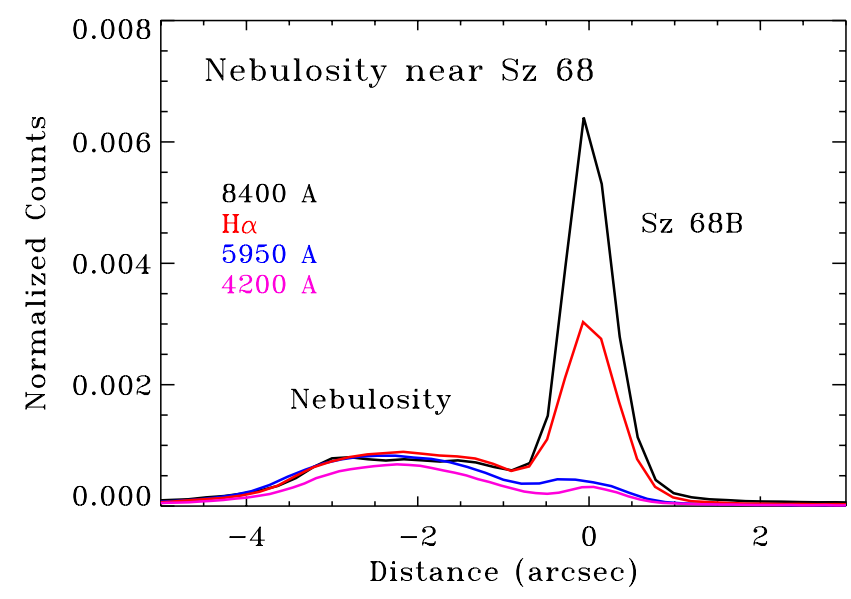

Figure 24. Shape of emission from $\mathrm{Sz} 68 \mathrm{~B}$ and the $\mathrm{Sz} 68$ A reflection nebulosity in the dispersion direction at four different wavelengths. Within the slit, nebulosity extends over $\sim 4^{\prime \prime}$ and reflects $1.5 \%$ of the total light from $\mathrm{Sz} 68 \mathrm{~A}$ at all optical wavelengths.

(A color version of this figure is available in the online journal.)

The $\mathrm{H}-\alpha$ emission indicates that GK Tau B is accreting, is a likely member of the Taurus Molecular Cloud, and is likely associated with GK Tau A.

\section{A.2. 2 MASS J04162709+2807313}

We decided to observe 2MASS J04162709+2807313 on a whim, since it is close (14") to $\mathrm{LkCa} 4$, had not been previously discussed in the literature, and was noticed to be bright during our acquisition of $\mathrm{LkCa} 4$. $\mathrm{LkCa} 4 \mathrm{~S}$ has a similar spectral type as $\mathrm{LkCa} 4 \mathrm{AB}$ but is a factor of 3.5 fainter. Weak $\mathrm{H} \alpha$ emission suggests chromospheric activity, an indicator of youth. The K I $7700 \AA$ and Na I $8200 \AA$ doublets are weak and indicate a low gravity, consistent with the pre-main-sequence. If this star is a wide binary companion to $\mathrm{LkCa} 4$, half of the luminosity difference is accounted for by the binarity of $\mathrm{LkCa} 4$.

The optical brightness of $\mathrm{LkCa} 4 \mathrm{~S}$ during our observation is surprising. The star is six magnitudes fainter than $\mathrm{LkCa} 4$ in 2MASS $J H K$ and in USNO-B, with one magnitude of variability. The $J H K$ color difference is consistent with $A_{V}=$ 1.9 mag. LkCa $4 \mathrm{~S}$ is also not listed in the WISE all sky catalogue, which rules out variability and extinction from an edge-on disk. Perhaps our observation occurred when the star peaked out of what is normally an opaque interstellar medium (ISM).

\section{A.3. 2MASS J16003440-4225386}

2MASS J16003440-4225386 was listed as a candidate member of Lupus based on an IR excess and colors that are consistent with a late M star (Chapman et al. 2007). The star has broad TiO bands but a huge absorption band around $8200 \AA$, characteristic of a late $\mathrm{M}$ star with a very low surface gravity. We classify the star as an $\sim$ M9 I supergiant and a likely Cepheid variable.

\section{A.4. Reflection Nebulosity of $\mathrm{Sz}, 68 \mathrm{~A}$}

$\mathrm{Sz} 68$ is a triple system, with the second and third components located 0"'126 and 2".808 from Sz 68 A (Correia et al. 2006). The star drives a bright jet, known as HH 186 (Heyer \& Graham 1989).

A bright reflection nebula was located between $\mathrm{Sz} 68 \mathrm{AB}$ and $\mathrm{Sz} 68 \mathrm{C}$ at the time of our observations. The spectrum of the reflection nebula is exactly the same as the $\mathrm{Sz} 68 \mathrm{AB}$ spectrum. Figure 24 shows the spatial profile of emission in 

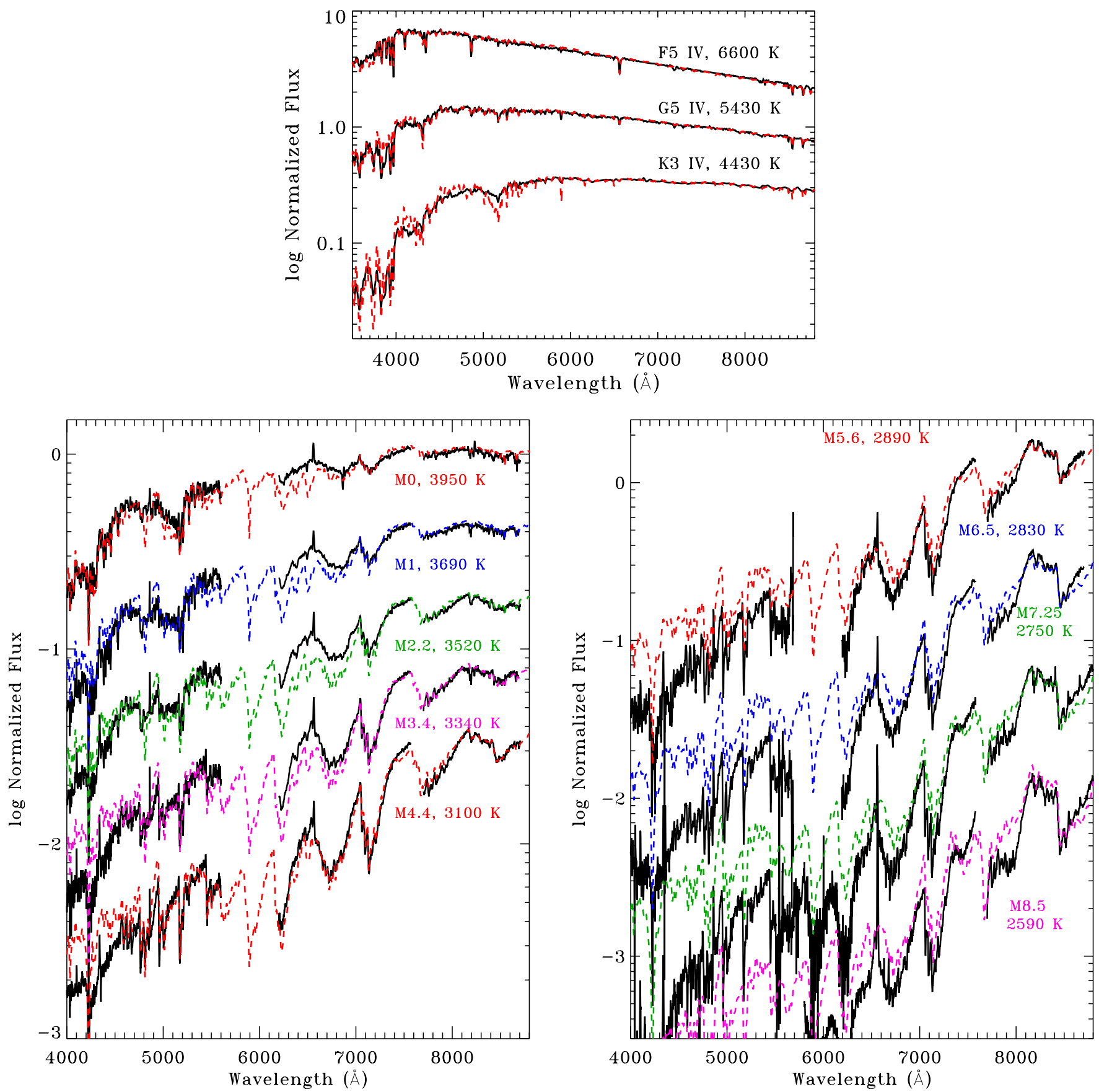

Figure 25. Top: comparisons between luminosity class IV stars in the Pickles library and the best-fit BT-Settl synthetic spectra, normalized at $7300 \AA \AA$. Bottom: comparisons between K5.5-M4 stars (left), M5-M8.5 stars (right, M8.5 is red only) from our grid of spectral templates (Table 4), and the best-fit BT-Settl synthetic spectra. Spectra are normalized at $7350 \AA$. The model spectra are then scaled by the best-fit parameter from Table 13. At spectral types later than M4, the blue spectra are much stronger in the BT-Settl models than in the observed spectra.

(A color version of this figure is available in the online journal.)

the slit of our Sz $68 \mathrm{C}$ observation. The nebulosity within our slit accounts for $1.5 \%$ of the total emission from $\mathrm{Sz} 68 \mathrm{AB}$. The total emission from the nebulosity is likely much higher. The fraction of light from Sz 68 AB scattered into our line of sight by the reflection nebulosity does not depend on wavelength. Extended dust emission was independently found in Herschel/PACS observations of the Sz 68 system (Cieza et al. 2013).

The Sz 68 C spectrum was extracted from the image by fitting a second-order polynomial to the edge of the nebulosity, subtracting off the model flux, and then extracting the leftover stellar flux.

\section{APPENDIX B}

\section{COMPARISONS BETWEEN SYNTHETIC AND OBSERVED SPECTRA}

This appendix and Figure 25 describe in detail our comparisons between observed spectra and the BT-Settl models with $\log g=4.0$ of Allard et al. (2012). The BT-Settl models in Allard et al. (2012) are calculated at $100 \mathrm{~K}$ intervals. Intermediate temperatures are calculated by linearly interpolating between temperatures at $10 \mathrm{~K}$ intervals. Our spectral type grid is listed in Table 4 and is supplemented with an M9.5 spectrum 
Table 13

Temperature Measurements for Spectral Type Grid

\begin{tabular}{|c|c|c|c|c|c|c|c|c|}
\hline Star & Date & $\mathrm{SpT}$ & Blue+Red & Red Only & Blue+Red, No TiO & TiO Only & Adopted & $\mathrm{Fac}$ \\
\hline HBC 407 & Dec 28 & K0 & 5110 & 4910 & $\ldots$ & 5100 & 5110 & 0.99 \\
\hline HBC 372 & Dec 28 & $\mathrm{~K} 2$ & 4710 & 4570 & $\ldots$ & 4850 & 4710 & 0.99 \\
\hline $\mathrm{LkCa} 14$ & Dec 28 & K5 & 4200 & 4240 & 4220 & 4540 & 4220 & 1.02 \\
\hline MBM12 1 & Jan 19 & K5.5 & 4160 & 4240 & 4190 & 4420 & 4190 & 0.99 \\
\hline TWA 9A & Jan 20 & K6.5 & 4140 & 4160 & 4160 & 4300 & 4160 & 1.03 \\
\hline V826 Tau & Dec 30 & K7 & 4020 & 4130 & 4020 & 4300 & 4020 & 1.05 \\
\hline V830 Tau & Dec 30 & K7.5 & 3940 & 4050 & 3930 & 4200 & 3930 & 1.03 \\
\hline TWA 6 & Dec 29 & M0 & 3930 & 3990 & 3910 & 4200 & 3910 & 1.05 \\
\hline TWA 25 & Dec 28 & M0.5 & 3840 & 3850 & 3850 & 3680 & 3770 & 1.05 \\
\hline TWA $13 \mathrm{~S}$ & Jan 18 & M1.0 & 3750 & 3810 & 3740 & 3620 & 3690 & 1.05 \\
\hline $\mathrm{LkCa} 4$ & Dec 30 & M1.5 & 3730 & 3800 & 3720 & 3620 & 3670 & 1.05 \\
\hline LkCa 5 & Dec 28 & M2.2 & 3550 & 3660 & 3530 & 3500 & 3520 & 1.05 \\
\hline LkCa 3 & Dec 28 & M2.4 & 3530 & 3650 & 3510 & 3500 & 3510 & 1.06 \\
\hline TWA 8A & Jan 18 & M3.0 & 3380 & 3530 & 3370 & 3410 & 3390 & 1.04 \\
\hline TWA 9B & Jan 20 & M3.4 & 3340 & 3440 & 3360 & 3330 & 3340 & 1.05 \\
\hline 2M J1207-3247 & Dec 28 & M3.5 & 3320 & 3430 & 3320 & 3380 & 3350 & 1.10 \\
\hline Hen 3-600 B & May 28 & M4.1 & 3140 & 3320 & 3140 & 3100 & 3120 & 1.05 \\
\hline XEST 16-045 & Dec 28 & M4.4 & 3070 & 3140 & 3100 & 3100 & 3100 & 1.06 \\
\hline J2 157 & Dec 28 & M4.7 & 3020 & 3080 & 3060 & 3040 & 3050 & 1.03 \\
\hline TWA 8B & Dec 30 & M5.2 & 2840 & 3000 & 2840 & 2990 & 2910 & 1.05 \\
\hline MBM12 7 & Dec 30 & M5.6 & 2830 & 2990 & 2830 & 2960 & 2890 & 1.05 \\
\hline V410 X-ray 3 & Dec 30 & M6.5 & 2770 & 2800 & 2780 & 2880 & 2830 & 1.09 \\
\hline Oph 1622 A & May 28 & M7.25 & 2680 & 2710 & 2680 & 2820 & 2750 & 1.08 \\
\hline
\end{tabular}

of KPNO 4 (K. L. Luhman 2008, private communication). The observed M8.5 and M9.5 spectra only cover red wavelengths (5700-9000 ̊).

For stars earlier than M0, synthetic spectra at some temperature can be found that reproduces the spectral shape and most features of the observed spectra. Some small differences occur at locations of strong lines, particularly the $\mathrm{MgH} / \mathrm{Mg}$ b complex at $5200 \AA$.

For stars later than M0, the synthetic spectra are less good at reproducing the observed young star spectra. Specifically, with normalization at $7300 \AA$, the synthetic spectra lack opacity and are much stronger than the observed spectra shortward of $5000 \AA$ A. This discrepancy increases toward cooler stars. The synthetic spectrum is also slightly fainter than the observed spectrum at 5500-6500 $\AA$. Later than $\sim \mathrm{M} 4$, the three strong TiO bands at 7140,7600 , and $8500 \AA$ in the synthetic spectra no longer match the observed band depth. The discrepancy at blue wavelengths also becomes larger. The $\mathrm{VO}$ absorption band at $7500 \AA$ is also much stronger in the synthetic spectra than in the observations, so the $7400-7600 \AA$ region is avoided in our temperature calculations.

The temperatures are calculated by considering both the overall SED and the absorption line and band strengths. Specifically, we find a synthetic spectrum and a normalization that best fits the observed spectra considering several ranges in wavelengths within the spectral range of the data: (a) for blue+red, fits to the full $4300-8700 \AA$ spectrum; (b) for red only, fits to the 6300-8700 A spectrum; (c) for the blue+red spectrum, where the fits exclude the spectral locations of deep TiO bands; and (d) for the depth of TiO bands, with each band normalized to a nearby wavelength region so that the TiO-only fit is independent of the broadband colors. Results from these fits are presented in Table 13. The adopted temperatures for (1) spectral types earlier than K5 are obtained from (a), the full blue+red fit; (2) for spectral types K5-M0, from (c), the blue+red spectrum that excludes $\mathrm{TiO}$ bands; and (3) for spectral types later than $\mathrm{M} 0$, the average temperature from (c), the blue+red fits excluding $\mathrm{TiO}$ and (d), the TiO-only fits. For M-dwarf models, the absolute scaling is based on the fit from (c), the blue+red without TiO, calculated for the adopted temperature. If the molecular data is insufficient to reproduce the depth of the strong molecular bands, then the fits that focus on continuum regions and exclude molecular bands may be more accurate. For stars earlier than M0, the fit to the full spectrum is used to convert $\mathrm{SpT}$ to temperature. For stars later than M0, the spectral locations outside of TiO bands and the depth of the TiO bands are averaged to provide our conversion to temperature. The scaling parameter between the observed and model spectra is also listed in Table 13 and is based on the 7350-7400 A spectral range.

The fits to the $\mathrm{TiO}$ spectrum and to the spectrum outside of the $\mathrm{TiO}$ bands differ by as much as $200 \mathrm{~K}$, which suggests a $\sim 100 \mathrm{~K}$ uncertainty in our conversion and that the model atmospheres do not yet reproduce spectra of pre-main-sequence stars that are cool enough for $\mathrm{TiO}$ and other molecules to provide significant opacity in the atmosphere. Increasing $\log g$ from 4.0 to 4.5 leads to a decrease of $\sim 50 \mathrm{~K}$ in temperature. The discrepancies between the synthetic and observed spectra are significant for objects later than M1, increase substantially at spectral types later than M4, and are especially large at M9.5. The uncertainty in the SpT-temperature conversion also leads to uncertainty in the bolometric corrections and in the luminosity from evolutionary models that use these atmospheres.

\section{APPENDIX C}

\section{TABLE OF STELLAR PROPERTIES}

Table 14 lists the abbreviated name, number of spectra $N$ on different nights, distance, spectral type, extinction, flux at $7510 \AA$ corrected for extinction, veiling $r$ at $7510 \AA$, and the $\log$ of the photospheric luminosity for all stars in our sample. An electronic-only table lists for each spectrum the full target name and position, the observation date, and mass and age estimates. Negative extinctions and the calculated extinctions to the TWA are listed in parenthesis and are treated as $A_{V}=0$ 
Table 14

Stellar Properties

\begin{tabular}{|c|c|c|c|c|c|c|c|}
\hline Object & $N$ & $\begin{array}{c}d \\
(\mathrm{pc})\end{array}$ & SpT & $\begin{array}{c}A_{V} \\
(\mathrm{mag})\end{array}$ & $\log _{\mathrm{a}} F$ & $\begin{array}{l}r \\
\mathrm{~b}\end{array}$ & $\begin{array}{l}\log L \\
\left(L_{\odot}\right)\end{array}$ \\
\hline MBM12 1 & 1 & 275 & K5.5 & 0.10 & -13.18 & 0.00 & 0.25 \\
\hline MBM12 2 & 1 & 275 & M0.3 & 1.65 & -13.83 & 0.18 & -0.39 \\
\hline MBM12 3 & 1 & 275 & M2.8 & 0.55 & -13.66 & 0.02 & -0.18 \\
\hline MBM12 7 & 1 & 275 & M5.6 & $(-0.10)$ & -14.79 & 0.00 & -1.14 \\
\hline LkHa 264 & 1 & 275 & K5.5 & $(-0.25)$ & -13.80 & 0.57 & -0.36 \\
\hline MBM12 8 & 1 & 275 & M5.9 & 0.30 & -15.19 & 0.00 & -1.51 \\
\hline MBM12 5 & 1 & 275 & $\mathrm{~K} 2.0$ & 0.90 & -12.96 & 0.00 & 0.44 \\
\hline MBM12 9 & 1 & 275 & M5.6 & 0.10 & -15.24 & 0.00 & -1.59 \\
\hline MBM12 6 & 1 & 275 & M3.8 & 0.50 & -14.23 & 0.04 & -0.70 \\
\hline MBM12 10 & 1 & 275 & M3.4 & 0.60 & -13.98 & 0.02 & -0.49 \\
\hline MBM12 11 & 1 & 275 & M5.8 & $(-0.10)$ & -15.07 & 0.01 & -1.40 \\
\hline MBM12 12 & 1 & 275 & M2.6 & 0.25 & -13.77 & 0.04 & -0.30 \\
\hline $2 \mathrm{M} 0325+2426$ & 1 & 140 & M4.4 & 0.80 & -14.44 & 0.00 & -1.46 \\
\hline c2d 0329+3118 & 1 & 315 & M0.0 & 3.50 & -14.08 & 0.04 & -0.53 \\
\hline c2d $0330+3032$ & 1 & 315 & M2.7 & 2.70 & -15.53 & 0.00 & -1.94 \\
\hline LkHa 329 & 1 & 315 & K5.0 & 2.70 & -13.19 & 0.11 & 0.36 \\
\hline LkHa 330 & 2 & 315 & F7.0 & 2.85 & -12.34 & 0.00 & 1.21 \\
\hline HBC 358 & 1 & 140 & M3.9 & 0.05 & -13.66 & 0.00 & -0.72 \\
\hline HBC 359 & 1 & 140 & M2.8 & $-0.25)$ & -13.65 & 0.00 & -0.76 \\
\hline HBC 360 & 1 & 140 & M3.4 & 0.30 & -13.78 & 0.00 & -0.87 \\
\hline HBC 361 & 1 & 140 & M3.2 & 0.40 & -13.75 & 0.00 & -0.85 \\
\hline HBC 362 & 1 & 140 & M2.7 & 0.10 & -13.84 & 0.00 & -0.95 \\
\hline $2 \mathrm{M} 0407+$ & 1 & 140 & M4.8 & 0.80 & -14.30 & 0.00 & -1.28 \\
\hline $\mathrm{LkCa} 1$ & 1 & 131 & M3.6 & 0.45 & -13 & 0.00 & -0.29 \\
\hline HBC 366 & 1 & 131 & M0.5 & 2.20 & -12.72 & 0.00 & 0.07 \\
\hline V773 Tau & 1 & 131 & $\mathrm{~K} 4.0$ & 0.95 & -12.30 & 0.00 & 0.48 \\
\hline FM Tau & 4 & 131 & M4.5 & 0.35 & -14.08 & 0.49 & -1.15 \\
\hline FN Tau & 4 & 131 & & & -13.14 & 0.02 & -0.28 \\
\hline CW Tau & 1 & 131 & K3.0 & 1.80 & -13.11 & 0.50 & -0.35 \\
\hline CIDA 1 & 1 & 131 & M4.5 & 3.00 & -13.65 & 0.13 & -0.72 \\
\hline MHO 3 & 1 & 132 & M2.2 & 5.30 & -13.63 & 0.00 & -0.81 \\
\hline FP Tau & 5 & 131 & M2.6 & & -13.61 & 0.05 & -0.78 \\
\hline XEST 20 & 1 & & M5.2 & $-0.14)$ & -13.98 & 0.00 & -1.00 \\
\hline CX Tau & 4 & 131 & M2.5 & 0.25 & -13.40 & 0.02 & -0.58 \\
\hline $\mathrm{LkCa} 3$ & 1 & 131 & M2.4 & 0.00 & -12.82 & 0.00 & -0.00 \\
\hline FO Tau & 4 & 131 & & 2.05 & -13.16 & 0.07 & -0.28 \\
\hline XEST 20-071 & 1 & & & & -13.04 & 0.00 & -0.21 \\
\hline $2 \mathrm{M} 0415+2818$ & 2 & 131 & M4.0 & 1.80 & -13.54 & 0.03 & -0.64 \\
\hline $2 \mathrm{M} \mathrm{0415+2909}$ & 1 & 131 & M0.6 & 2.80 & -13.25 & 0.00 & -0.46 \\
\hline $2 \mathrm{M} 0415+2746$ & 1 & 140 & M5.2r & 0.60 & -14.34 & 0.00 & -1.30 \\
\hline $2 \mathrm{M} \mathrm{0416+2807}$ & 1 & & & $-0.15)$ & -13.77 & 0.00 & -0.97 \\
\hline $\mathrm{LkCa} 4$ & 2 & 131 & M1.3 & 0.35 & -13.09 & 0.00 & -0.29 \\
\hline CY Tau & 4 & 131 & M2.3 & 0.35 & -13.39 & 0.13 & -0.58 \\
\hline $\mathrm{LkCa} 5$ & 1 & 131 & M2.2 & 0.05 & -13.46 & 0.00 & -0.64 \\
\hline V410 X-r & 1 & & M3.7 & 1.70 & -14.42 & 0.04 & -1.55 \\
\hline V410 X-ray 3 & 1 & 131 & M6.5 & 0.20 & -14.54 & 0.00 & -1.45 \\
\hline V409 Tau & 3 & 131 & M0.6 & 1.00 & -12.98 & 0.00 & -0.18 \\
\hline HBC 372 & 1 & 147 & $\mathrm{~K} 2.0$ & 0.65 & -13.55 & 0.00 & -0.69 \\
\hline KPNO 11 & 1 & 131 & M5.9 & $-0.20)$ & -14.50 & 0.00 & -1.47 \\
\hline DD Tau & 1 & 131 & M4.8 & 0.75 & -13.50 & 0.44 & -0.54 \\
\hline CZ Tau & 2 & 131 & M4.2 & 0.50 & -13.67 & 0.00 & -0.76 \\
\hline V892 Tau & 1 & 131 & A0 & $9.30^{\mathrm{c}}$ & -10.72 & 0.00 & 2.11 \\
\hline Hubble 4 & 1 & 131 & K8.5 & 1.35 & -12.75 & 0.00 & 0.04 \\
\hline HBC 376 & 1 & 147 & $\mathrm{~K} 4.0$ & 0.25 & -13.22 & 0.00 & -0.34 \\
\hline V410 X-ray 6 & 2 & 131 & M5.9 & 1.40 & -13.95 & 0.00 & -0.92 \\
\hline FQ Tau & 1 & 131 & M4.3 & 1.60 & -13.45 & 0.15 & -0.54 \\
\hline BP Tau & 4 & 131 & M0.5 & 0.45 & -13.17 & 0.32 & -0.38 \\
\hline V819 Tau & 1 & 131 & K8.0 & 1.00 & -13.11 & 0.00 & -0.32 \\
\hline FR Tau & 1 & 131 & M5.3 & 0.20 & -14.03 & 0.03 & -1.04 \\
\hline $\mathrm{LkCa} 7$ & 1 & 131 & M1.2 & 0.05 & -13.14 & 0.00 & -0.34 \\
\hline $2 \mathrm{M} \mathrm{0420+2804}$ & 1 & 131 & M3.5 & 0.25 & -13.66 & 0.00 & -0.80 \\
\hline XEST 16-045 & 1 & 131 & M4.5 & $(-0.05)$ & -13.80 & 0.00 & -0.87 \\
\hline J2 157 & 1 & 131 & M4.6 & 0.35 & -14.13 & 0.00 & -1.19 \\
\hline IRAS 04187+1927 & 1 & 131 & M2.4 & 3.10 & -13.38 & 0.08 & -0.56 \\
\hline DE Tau & 4 & 131 & M2.3 & 0.35 & -13.09 & 0.05 & -0.28 \\
\hline
\end{tabular}

Table 14

(Continued)

\begin{tabular}{|c|c|c|c|c|c|c|c|}
\hline Object & $N$ & $\begin{array}{c}d \\
(\mathrm{pc})\end{array}$ & SpT & $\begin{array}{c}A_{V} \\
(\mathrm{mag})\end{array}$ & $\log _{\mathrm{a}} F$ & $\begin{array}{l}r \\
\mathrm{~b}\end{array}$ & $\begin{array}{l}\log L \\
\left(L_{\odot}\right)\end{array}$ \\
\hline RY Tau & 1 & 131 & G0 & 1.85 & -11.74 & 0.00 & 1.03 \\
\hline HD 283572 & 1 & 131 & G4 & 0.50 & -12.00 & 0.00 & 0.76 \\
\hline T Tau & 1 & 147 & K0 & 1.25 & -12.01 & 0.10 & 0.85 \\
\hline FS Tau & 1 & 131 & M2.4 & 2.95 & -13.66 & 0.46 & -0.84 \\
\hline LkCa 21 & 1 & 140 & M2.5 & 0.30 & -13.25 & 0.00 & -0.37 \\
\hline XEST 11-078 & 1 & 140 & M0.7 & 1.55 & -14.90 & 0.00 & -2.04 \\
\hline CFHT 21 & 1 & 140 & M1.5 & 3.75 & -14.13 & 0.27 & -1.27 \\
\hline FU Tau & 1 & 140 & M6.5 & 1.20 & -14.14 & 0.01 & -1.00 \\
\hline FT Tau & 1 & 140 & M2.8 & 1.30 & -13.64 & 0.27 & -0.75 \\
\hline IRAS $04216+2603$ & 1 & 140 & M2.8 & 1.90 & -13.95 & 0.37 & -1.07 \\
\hline $\mathrm{J} 4423$ & 1 & 140 & M4.5 & 0.25 & -14.07 & 0.00 & -1.08 \\
\hline IP Tau & 1 & 140 & M0.6 & 0.75 & -13.26 & 0.12 & -0.41 \\
\hline J4872 A & 1 & 140 & M0.6 & 1.20 & -13.20 & 0.00 & -0.35 \\
\hline J4872 B & 1 & 140 & M3.7 & 1.60 & -13.51 & 0.00 & -0.58 \\
\hline FV Tau A & 1 & 140 & M0.0 & 4.30 & -13.33 & 0.21 & -0.48 \\
\hline FV Tau B & 1 & 140 & M3.8 & 3.10 & -13.65 & 0.07 & -0.71 \\
\hline KPNO 13 & 1 & 140 & M5.1 & 1.80 & -14.06 & 0.00 & -1.02 \\
\hline DF Tau & 2 & 140 & M2.7 & 0.10 & -12.92 & 0.16 & -0.04 \\
\hline DG Tau & 4 & 140 & K7.0 & 1.60 & -13.14 & 0.40 & -0.29 \\
\hline HBC 388 & 1 & 140 & G6.0 & 0.25 & -12.53 & 0.00 & 0.27 \\
\hline J507 & 1 & 140 & M4.2 & 0.50 & -13.38 & 0.00 & -0.42 \\
\hline FW Tau & 1 & 140 & M5.8 & $(-0.20)$ & -13.88 & 0.00 & -0.80 \\
\hline GV Tau & 2 & 140 & $\mathrm{c}$ & 3.91 & -13.90 & $\ldots$ & $\ldots$ \\
\hline XEST 15-034 & 1 & 140 & M4.1 & 0.20 & -14.11 & 0.00 & -1.15 \\
\hline DH Tau & 1 & 140 & M2.3 & 0.65 & -13.53 & 0.40 & -0.66 \\
\hline DI Tau & 2 & 140 & M0.7 & 0.70 & -13.01 & 0.00 & -0.16 \\
\hline IQ Tau & 2 & 140 & M1.1 & 0.85 & -13.47 & 0.11 & -0.61 \\
\hline CFHT 20 & 1 & 140 & M5.2 & 2.30 & -14.27 & 0.00 & -1.23 \\
\hline UX Tau W & 2 & 140 & M1.9 & 0.40 & -13.30 & 0.00 & -0.43 \\
\hline UX Tau E & 2 & 140 & K0.0 & 0.65 & -12.61 & 0.00 & 0.20 \\
\hline UX Tau C & 1 & 140 & M2.8 & $(-0.05)$ & -14.12 & 0.00 & -1.24 \\
\hline FX Tau & 1 & 140 & M2.2 & 0.80 & -13.16 & 0.06 & -0.29 \\
\hline DK Tau A & 1 & 140 & $\mathrm{~K} 8.5 \mathrm{c}$ & 0.70 & -13.12 & 0.27 & -0.27 \\
\hline DK Tau B & 2 & 140 & M1.7 & 1.80 & -13.62 & 0.28 & -0.76 \\
\hline ZZ Tau & 4 & 140 & M4.3 & 0.55 & -13.25 & 0.02 & -0.28 \\
\hline ZZ Tau IRS & 1 & 140 & M4.5 & 1.70 & -14.65 & 0.10 & -1.66 \\
\hline JH 56 & 1 & 140 & K8.0 & 0.35 & -13.17 & 0.00 & -0.32 \\
\hline V927 Tau & 1 & 140 & M4.9 & $(-0.20)$ & -13.53 & 0.00 & -0.51 \\
\hline LkHa 358 & 1 & 140 & M0.9 & 2.80 & -14.80 & 0.35 & -1.94 \\
\hline HL Tau & 1 & 140 & $\mathrm{~K} 3 \mathrm{c}$ & 2.50 & -13.67 & 0.47 & -0.84 \\
\hline XZ Tau & 1 & 140 & M3.2 & 1.50 & -13.14 & 0.07 & -0.24 \\
\hline HK Tau & 3 & 140 & M1.5 & 2.40 & -13.39 & 0.10 & -0.52 \\
\hline V710 Tau A & 2 & 140 & M3.3 & 0.80 & -13.34 & 0.00 & -0.43 \\
\hline V710 Tau B & 1 & 140 & M1.7 & 0.55 & -13.47 & 0.03 & -0.61 \\
\hline $\mathrm{J} 665$ & 1 & 140 & M4.9 & 0.40 & -13.84 & 0.00 & -0.81 \\
\hline V1075 Tau & 1 & 140 & K6.0 & 0.25 & -13.12 & 0.00 & -0.28 \\
\hline V827 Tau & 1 & 140 & M1.4 & 0.05 & -13.29 & 0.00 & -0.43 \\
\hline Haro 6-13E & 1 & 140 & M1.6 & 2.20 & -13.43 & 0.41 & -0.57 \\
\hline Haro 6-13W & 1 & 140 & K5.5 & 2.25 & -12.89 & 0.13 & -0.04 \\
\hline V826 Tau & 1 & 140 & K7.0 & 0.40 & -13.04 & 0.00 & -0.20 \\
\hline MHO 5 & 1 & 140 & M6.5 & $(-0.20)$ & -14.13 & 0.00 & -0.99 \\
\hline CFHT 7 & 1 & 140 & M6.7 & 0.20 & -14.61 & 0.00 & -1.44 \\
\hline V928 Tau & 1 & 140 & M0.8 & 1.95 & -13.03 & 0.00 & -0.18 \\
\hline MHO 6 & 1 & 140 & M5.0 & $(-0.15)$ & -14.25 & 0.01 & -1.22 \\
\hline MHO 7 & 1 & 140 & M5.3 & $(-0.20)$ & -14.16 & 0.00 & -1.12 \\
\hline GG Tau B & 2 & 140 & M5.8 & 0.00 & -14.21 & 0.00 & -1.13 \\
\hline GG Tau A & 2 & 140 & K7.5 & 1.00 & -12.69 & 0.07 & 0.15 \\
\hline FY Tau & 2 & 140 & M0.1 & 3.05 & -13.24 & 0.16 & -0.39 \\
\hline FZ Tau & 3 & 140 & M0.5c & 3.50 & -13.33 & 0.30 & -0.48 \\
\hline UZ Tau B & 1 & 140 & M3.1 & 0.70 & -13.10 & 0.03 & -0.21 \\
\hline UZ Tau A & 1 & 140 & M1.9 & 0.90 & -13.26 & 0.14 & -0.40 \\
\hline HBC 403 & 1 & 140 & K6.0 & 0.85 & -13.20 & 0.00 & -0.36 \\
\hline JH 112A & 4 & 140 & K5.5 & 3.10 & -13.27 & 0.00 & -0.42 \\
\hline JH 112B & 1 & 140 & M4.6 & 2.95 & -13.99 & 0.00 & -0.99 \\
\hline GH Tau & 1 & 140 & M2.3 & 0.40 & -13.07 & 0.00 & -0.19 \\
\hline
\end{tabular}


Table 14

(Continued)

\begin{tabular}{|c|c|c|c|c|c|c|c|}
\hline Object & $N$ & $\begin{array}{c}d \\
(\mathrm{pc})\end{array}$ & SpT & $\begin{array}{c}A_{V} \\
(\mathrm{mag})\end{array}$ & $\underset{\mathrm{a}}{\log F}$ & $\begin{array}{l}r \\
\mathrm{~b}\end{array}$ & $\begin{array}{l}\log L \\
\left(L_{\odot}\right)\end{array}$ \\
\hline V807 Tau & 1 & 140 & K7.5 & 0.50 & -12.58 & 0.05 & 0.26 \\
\hline V830 Tau & 1 & 140 & K7.5 & 0.45 & -13.09 & 0.00 & -0.24 \\
\hline GI Tau & 3 & 140 & M0.4 & $2.05^{\mathrm{d}}$ & -13.10 & 0.04 & -0.25 \\
\hline GK Tau A & 5 & 140 & K6.5 & $1.50^{\mathrm{d}}$ & -12.87 & 0.08 & -0.03 \\
\hline GK Tau B & 1 & 140 & K3.0 & 2.20 & -14.01 & 0.00 & -1.18 \\
\hline IS Tau & 1 & 140 & M2.0 & 2.55 & -13.26 & 0.02 & -0.39 \\
\hline DL Tau & 4 & 140 & $\mathrm{~K} 5.5 \mathrm{c}$ & 1.80 & -13.15 & 0.36 & -0.30 \\
\hline HN Tau A & 1 & 140 & $\mathrm{~K} 3 \mathrm{c}$ & 1.15 & -13.60 & 0.49 & -0.77 \\
\hline HN Tau B & 1 & 140 & M4.8r & 0.60 & -14.67 & 0.00 & -1.65 \\
\hline $2 \mathrm{M} 0433+2615$ & 1 & 140 & M5.2r & 3.20 & -14.14 & 0.00 & -1.10 \\
\hline DM Tau & 4 & 140 & M3.0 & 0.10 & -13.78 & 0.12 & -0.89 \\
\hline CI Tau & 1 & 140 & K5.5 & 1.90 & -13.05 & 0.40 & -0.20 \\
\hline XEST 17-059 & 1 & 161 & M5.5 & 1.00 & -13.75 & 0.00 & -0.57 \\
\hline IT Tau A & 2 & 140 & K6.0 & 3.10 & -12.86 & 0.00 & -0.01 \\
\hline IT Tau B & 1 & 140 & M2.9r & 5.60 & -13.21 & 0.00 & -0.32 \\
\hline J2 2041 & 1 & 140 & M3.7 & 0.45 & -13.61 & 0.00 & -0.68 \\
\hline JH 108 & 1 & 161 & M1.5 & 1.75 & -13.50 & 0.00 & -0.51 \\
\hline НBC 407 & 1 & 140 & K0 & 0.80 & -13.26 & 0.00 & -0.45 \\
\hline AA Tau & 3 & 140 & M0.6 & 0.40 & -13.20 & 0.03 & -0.35 \\
\hline HO Tau & 1 & 161 & M3.2 & 1.00 & -13.87 & 0.20 & -0.85 \\
\hline FF Tau & 1 & 161 & $\mathrm{~K} 8.0$ & 2.00 & -13.00 & 0.00 & -0.04 \\
\hline HBC 412 & 1 & 140 & M2.6 & 0.30 & -13.39 & 0.00 & -0.51 \\
\hline DN Tau & 1 & 140 & M0.3 & 0.55 & -12.93 & 0.00 & -0.08 \\
\hline CoKu Tau 3A & 1 & 140 & M0.5 & 3.40 & -13.72 & 0.00 & -0.87 \\
\hline CoKu Tau 3B & 1 & 140 & M4.3r & 6.70 & -13.78 & 0.00 & -0.80 \\
\hline HQ Tau & 1 & 161 & $\mathrm{~K} 2.0$ & 2.60 & -12.29 & 0.00 & 0.65 \\
\hline HP Tau & 1 & 161 & $\mathrm{~K} 4.0$ & 3.15 & -12.92 & 0.16 & 0.03 \\
\hline HP Tau G3 & 1 & 161 & M0.6r & 2.10 & -13.32 & 0.00 & -0.35 \\
\hline HP Tau G2 & 3 & 161 & G2 & 2.55 & -12.09 & 0.00 & 0.84 \\
\hline Haro 6-28 & 1 & 161 & M3.1 & 2.85 & -13.52 & 0.15 & -0.50 \\
\hline XEST 09-042 & 1 & 161 & K7.0 & 1.05 & -12.90 & 0.05 & 0.06 \\
\hline LkCa 14 & 1 & 140 & K5.0 & 0.00 & -13.00 & 0.00 & -0.15 \\
\hline $2 \mathrm{M} 0436+2351$ & 1 & 140 & M5.1 & $-0.20)$ & -14.95 & 0.01 & -1.91 \\
\hline GM Tau & 1 & 140 & M5.0 & 2.10 & -14.42 & 0.26 & -1.38 \\
\hline DO Tau & 2 & 140 & M0.3 & $0.75^{\mathrm{c}}$ & -13.49 & 0.54 & -0.64 \\
\hline HV Tau & 1 & 140 & M4.1 & 1.40 & -13.14 & 0.00 & -0.18 \\
\hline $2 \mathrm{M} \mathrm{0439+2336}$ & 1 & 140 & M4.9 & $(-0.20)$ & -14.07 & 0.01 & -1.05 \\
\hline VY Tau & 1 & 161 & M1.5 & 0.60 & -13.39 & 0.02 & -0.41 \\
\hline LkCa 15 & 4 & 161 & K5.5 & 0.30 & -13.06 & 0.04 & -0.09 \\
\hline GN Tau & 2 & 140 & M2.5 & 3.05 & -13.33 & 0.12 & -0.45 \\
\hline ITG 15 & 1 & 140 & M5.0 & 2.65 & & & -0.61 \\
\hline JH 223 & 1 & 140 & M2.8 & 1.20 & -13.65 & 0.00 & -0.76 \\
\hline Haro 6-32 & 1 & 140 & M5.2 & 0.75 & -13.95 & 0.00 & -0.91 \\
\hline IW Tau & 1 & 140 & M0.9 & 0.40 & -13.13 & 0.00 & -0.28 \\
\hline CoKu Tau 4 & 2 & & & & -13.35 & 0.00 & -0.50 \\
\hline $2 \mathrm{M} 0441+2301$ & 1 & 140 & M4.3 & $(-0.15)$ & -13.82 & 0.00 & -0.85 \\
\hline HBC 422 & 1 & 140 & M0.6 & 2.60 & -13.08 & 0.00 & -0.23 \\
\hline НBC 423 & 1 & 140 & M2.5 & 2.65 & -13.02 & 0.00 & -0.14 \\
\hline V955 Tau & 2 & 140 & K8.5 & 2.90 & -13.20 & 0.06 & -0.35 \\
\hline CIDA 7 & 1 & 140 & M5.1 & 1.10 & -14.13 & 0.04 & -1.09 \\
\hline DP Tau & 3 & 140 & M0.8c & 0.80 & -14.04 & 0.38 & -1.18 \\
\hline GO Tau & 5 & 140 & M2.3 & 1.50 & -13.57 & 0.09 & -0.70 \\
\hline CIDA 14 & 2 & 140 & M5.5 & $(-0.20)$ & -13.89 & 0.00 & -0.84 \\
\hline RX J0446.7+2459 & 1 & 140 & M5.5 & 0.00 & -14.25 & 0.00 & -1.19 \\
\hline DQ Tau & 1 & 140 & M0.6 & 1.40 & -13.08 & 0.06 & -0.23 \\
\hline Haro 6-37A & 1 & 140 & K8.0 & 2.05 & -13.97 & 0.33 & -1.13 \\
\hline Haro 6-37B & 1 & 140 & M0.9 & 0.85 & -13.93 & 0.36 & -1.07 \\
\hline DR Tau & 4 & 140 & K6c & 0.45 & -13.33 & 0.51 & -0.49 \\
\hline DS Tau & 4 & 140 & M0.4 & 0.25 & -13.57 & 0.36 & -0.72 \\
\hline UY Aur & 1 & 140 & K7.0 & 1.00 & -12.91 & 0.07 & -0.07 \\
\hline ST 34 & 1 & 140 & M3.4 & 0.50 & -13.70 & 0.14 & -0.79 \\
\hline GM Aur & 5 & 140 & K6.0 & 0.30 & -13.16 & 0.18 & -0.31 \\
\hline LkCa 19 & 1 & 140 & $\mathrm{~K} 2.0$ & 0.50 & -12.69 & 0.00 & 0.13 \\
\hline $2 \mathrm{M} 0455+3019$ & 1 & 140 & M4.7 & 0.70 & -14.00 & 0.01 & -0.99 \\
\hline AB Aur & 1 & 140 & A 1.0 & 0.55 & -11.49 & 0.00 & 1.39 \\
\hline
\end{tabular}

Table 14

(Continued)

\begin{tabular}{|c|c|c|c|c|c|c|c|}
\hline Object & $N$ & $\begin{array}{c}d \\
(\mathrm{pc})\end{array}$ & SpT & $\begin{array}{c}A_{V} \\
(\mathrm{mag})\end{array}$ & $\log _{\mathrm{a}} F$ & $\begin{array}{l}r \\
\mathrm{~b}\end{array}$ & $\begin{array}{l}\log L \\
\left(L_{\odot}\right)\end{array}$ \\
\hline $2 \mathrm{M} 0455+3028$ & 1 & 140 & M5.0 & 0.20 & -14.10 & 0.00 & -1.06 \\
\hline XEST 26-062 & 1 & 140 & M4.0 & 0.85 & -13.68 & 0.01 & -0.73 \\
\hline SU Aur & 1 & 140 & G4 & 0.70 & -12.02 & 0.00 & 0.79 \\
\hline НBC 427 & 1 & 140 & K6.0 & 0.20 & -12.90 & 0.00 & -0.05 \\
\hline V836 Tau & 1 & 140 & M0.8 & 0.60 & -13.37 & 0.02 & -0.52 \\
\hline CIDA 8 & 1 & 140 & M3.7 & 1.70 & -13.71 & 0.10 & -0.78 \\
\hline CIDA 9A & 1 & 140 & M1.8 & 1.35 & -13.74 & 0.19 & -0.88 \\
\hline CIDA 9B & 1 & 140 & M4.6r & 0.05 & -14.00 & 0.00 & -1.00 \\
\hline CIDA 10 & 1 & 140 & M4.2 & 0.55 & -13.74 & 0.00 & -0.77 \\
\hline CIDA 11 & 1 & 140 & M4.2 & 0.35 & -13.58 & 0.05 & -0.61 \\
\hline $2 \mathrm{M} 0506+2104$ & 1 & 140 & M5.6 & $(-0.20)$ & -14.65 & 0.00 & -1.59 \\
\hline RW Aur B & 1 & 140 & K6.5 & 0.10 & -13.20 & 0.18 & -0.36 \\
\hline RW Aur A & 1 & 140 & K0 & $(-0.25)$ & -12.95 & 0.52 & -0.14 \\
\hline CIDA 12 & 1 & 140 & M3.7 & 0.50 & -14.00 & 0.03 & -1.07 \\
\hline $2 \mathrm{M} 0516+2214$ & 1 & 140 & M5.0 & $(-0.10)$ & -14.35 & 0.00 & -1.32 \\
\hline $2 \mathrm{M} 0518+2327$ & 1 & 140 & M5.2 & $(-0.00)$ & -14.96 & 0.12 & -1.91 \\
\hline CVSO 224 & 1 & 416 & M3.5 & 0.40 & -14.66 & 0.01 & -0.80 \\
\hline $2 \mathrm{M} 0532+2423$ & 1 & 140 & M6.0 & $(-0.15)$ & -15.19 & 0.00 & -2.09 \\
\hline $2 \mathrm{M} 0537+2428$ & 2 & 140 & M5.5 & $(-0.25)$ & -14.35 & 0.00 & -1.29 \\
\hline $2 \mathrm{M} \mathrm{0539+2322}$ & 1 & 140 & M5.8 & $(-0.25)$ & -14.82 & 0.00 & -1.73 \\
\hline RR Tau & 1 & $\ldots$ & A3 & 2.05 & -12.84 & 0.00 & $\ldots$ \\
\hline $2 \mathrm{M} 0542+2213$ & 1 & 140 & M2.8 & 0.20 & -13.81 & 0.00 & -0.92 \\
\hline AT Pyx & 1 & 400 & $\mathrm{~K} 2.0$ & 1.20 & -13.45 & 0.00 & 0.28 \\
\hline TWA 6 & 2 & 67 & M0.0 & $(0.05)$ & -12.86 & 0.00 & -0.66 \\
\hline TWA 7 & 2 & 34 & M3.2 & $(-0.10)$ & -12.61 & 0.00 & -0.94 \\
\hline TWA 1 & 7 & 54 & M0.5 & 0.00 & -12.75 & 0.15 & -0.72 \\
\hline TWA 2 & 1 & 42 & M2.2 & $(-0.15)$ & -12.50 & 0.00 & -0.67 \\
\hline TWA 3A & 1 & 35 & M4.1 & $(0.05)$ & -12.68 & 0.01 & -0.92 \\
\hline TWA 3B & 1 & 35 & M4.0 & $(0.20)$ & -12.85 & 0.00 & -1.10 \\
\hline TWA 14 & 1 & 96 & M1.9 & $(0.10)$ & -13.12 & 0.00 & -0.58 \\
\hline TWA $13 \mathrm{~N}$ & 2 & 56 & M1.1 & $(0.20)$ & -12.78 & 0.00 & -0.71 \\
\hline TWA $13 \mathrm{~S}$ & 2 & 59 & M1.0 & $(0.15)$ & -12.77 & 0.00 & -0.66 \\
\hline TWA 4 & 1 & 45 & K6.0 & $(0.10)$ & -11.84 & 0.00 & 0.02 \\
\hline TWA 5 & 1 & 49 & M2.7 & $(-0.20)$ & -12.58 & 0.00 & -0.61 \\
\hline TWA 8B & 4 & 39 & M5.2 & $(0.20)$ & -13.60 & 0.00 & -1.66 \\
\hline TWA 8A & 3 & 43 & M2.9 & $(0.05)$ & -12.82 & 0.00 & -0.96 \\
\hline TWA 9B & 1 & 52 & M3.4 & 0.00 & -13.43 & 0.00 & -1.38 \\
\hline TWA 9A & 1 & 47 & K6.0 & $(-0.05)$ & -12.73 & 0.00 & -0.83 \\
\hline TWA 23 & 1 & 49 & M3.5 & $(0.05)$ & -12.91 & 0.00 & -0.91 \\
\hline TWA 25 & 1 & 54 & M0.5 & $(0.05)$ & -12.68 & 0.00 & -0.65 \\
\hline HR 4796 & 1 & 73 & A0 & 0.00 & -11.13 & 0.00 & 1.20 \\
\hline Sz 65 & 1 & 150 & K6.0 & 0.80 & -12.95 & 0.00 & -0.05 \\
\hline Sz 66 & 1 & 150 & M4.3 & 0.50 & -13.77 & 0.04 & -0.73 \\
\hline $\mathrm{Sz} 68 \mathrm{~A}$ & 1 & 150 & $\mathrm{~K} 2.0$ & 1.00 & -12.14 & 0.00 & 0.74 \\
\hline Sz 68 B & 1 & 150 & M5.9 & $(-0.10)$ & -14.19 & 0.00 & -1.04 \\
\hline GW Lup & 1 & 150 & M2.3 & 0.55 & -13.57 & 0.08 & -0.63 \\
\hline HM Lup & 1 & 150 & M2.9 & 0.60 & -13.75 & 0.10 & -0.80 \\
\hline Sz 73 & 1 & 150 & K8.5 & 2.75 & -13.67 & 0.36 & -0.77 \\
\hline GQ Lup & 1 & 150 & K5.0 & 1.60 & -12.95 & 0.35 & -0.04 \\
\hline $\mathrm{Sz} 76$ & 1 & 150 & M3.2 & 0.90 & -13.69 & 0.03 & -0.73 \\
\hline Sz 77 & 1 & 150 & K5.5 & 0.70 & -13.04 & 0.06 & -0.14 \\
\hline Sz 81A & 1 & 150 & M4.4 & 0.05 & -13.84 & 0.01 & -0.80 \\
\hline Sz 81B & 1 & 150 & M5.1 & $(-0.10)$ & -14.11 & 0.00 & -1.01 \\
\hline RX J1556.1-3655 & 1 & 150 & M1.2 & 0.60 & -13.63 & 0.26 & -0.71 \\
\hline IM Lup & 1 & 150 & K6.0 & 0.40 & -12.94 & 0.00 & -0.03 \\
\hline Sz 84 & 1 & 150 & M4.4 & 0.80 & -13.89 & 0.04 & -0.85 \\
\hline UScoCTIO 33 & 1 & 145 & M4.5 & 0.40 & -14.60 & 0.25 & -1.58 \\
\hline HD 143006 & 2 & 145 & G3 & 0.45 & -12.45 & 0.00 & 0.39 \\
\hline $2 \mathrm{M} 1558-1758$ & 1 & 145 & K5.0 & 0.20 & -13.06 & 0.00 & -0.18 \\
\hline UScoCTIO 128 & 1 & 145 & M6.2 & 0.50 & -15.61 & 0.03 & -2.46 \\
\hline UScoCTIO 112 & 1 & 145 & M5.1 & 0.75 & -15.02 & 0.00 & -1.94 \\
\hline UScoCTIO 100 & 1 & 145 & M5.7 & 0.40 & -14.93 & 0.00 & -1.83 \\
\hline 2M 1605-1933 & 1 & 145 & M4.4 & 1.60 & -14.39 & 0.16 & -1.38 \\
\hline 2M 1606-2056 & 1 & 145 & M6.9 & 1.00 & -15.29 & 0.01 & -2.08 \\
\hline Sz 91 & 1 & 200 & M2.0 & 1.60 & -13.74 & 0.10 & -0.56 \\
\hline
\end{tabular}


Table 14

(Continued)

\begin{tabular}{lccccccc}
\hline \hline Object & $N$ & $\begin{array}{c}d \\
(\mathrm{pc})\end{array}$ & SpT & $\begin{array}{c}A_{V} \\
(\mathrm{mag})\end{array}$ & $\begin{array}{c}\log F \\
\mathrm{a}\end{array}$ & $\begin{array}{c}r \\
\mathrm{~b}\end{array}$ & $\begin{array}{c}\log L \\
\left(L_{\odot}\right)\end{array}$ \\
\hline Sz 96 & 1 & 200 & $\mathrm{M} 0.8$ & 0.95 & -13.39 & 0.07 & -0.23 \\
Sz 98 & 1 & 200 & M0.4 & 1.25 & -13.42 & 0.33 & -0.26 \\
Sz 102 & 1 & 200 & $\mathrm{c}$ & 0 & -15.35 & $\ldots$ & $\ldots$ \\
Sz 104 & 1 & 200 & M4.6 & 0.85 & -14.15 & 0.04 & -0.84 \\
Sz 111 & 1 & 200 & M1.2 & 0.85 & -13.64 & 0.05 & -0.48 \\
AS 205 B & 1 & 121 & M0.1 & 2.40 & -12.67 & 0.32 & 0.05 \\
AS 205 A & 1 & 121 & $\mathrm{c}$ & 1.75 & -12.38 & $\ldots$ & $\ldots$ \\
SST Lup3 1 & 1 & 200 & M4.9 & 0.85 & -14.44 & 0.04 & -1.10 \\
2M 1614-2305 & 1 & 121 & K4.0 & 0.40 & -12.50 & 0.00 & 0.21 \\
V892 Sco & 1 & 121 & K2.0 & 0.90 & -13.79 & 0.36 & -1.10 \\
DoAr 21 & 1 & 121 & G1 & 7.10 & -11.46 & 0.00 & 1.23 \\
SR 21 & 2 & 121 & F7 & 6.20 & -11.84 & 0.00 & 0.87 \\
SR 21 B & 1 & 121 & M3.6r & 5.40 & -13.89 & 0.00 & -1.09 \\
IRS 48 & 1 & 121 & A0r & 11.35 & -12.11 & 0.00 & 0.66 \\
SR 9 & 2 & 121 & K6.0 & 0.25 & -12.86 & 0.10 & -0.14 \\
RNO 91 & 1 & 121 & K3c & 3.10 & -12.88 & 0.45 & -0.18 \\
RXJ 1842.9-3532 & 1 & 130 & K3.0 & 0.60 & -13.13 & 0.11 & -0.37 \\
RXJ 1852.3-3700 & 1 & 130 & K4.0 & 0.25 & -13.26 & 0.18 & -0.49 \\
DG CrA & 1 & 130 & K5.0 & 1.0 & -13.30 & 0.14 & -0.52 \\
HBC 680 & 1 & 130 & M1.9 & 1.50 & -13.03 & 0.00 & -0.23 \\
VV CrA & 1 & 130 & $\mathrm{c}$ & 3.95 & -12.67 & $\ldots$ & $\ldots$ \\
\hline
\end{tabular}

Notes.

a $F$ : photospheric flux at $7510 \AA \mathrm{erg} \mathrm{cm}^{-2} \mathrm{~s}^{-1} \AA^{-1}$.

b $r$ : veiling at $7510 \AA$.

c $R_{V}=5.5$ for V892 Tau.

d Variable $A_{V}$, average listed here.

e $R_{V}=4$ for DoAr 21 and SR 21 and 5.5 for IRS 48 .

(This table is also available in a machine-readable form in the online journal.)

when calculating luminosities. Several stars only have red spectra because of either detector failure or the star was too faint, and are noted with " $r$ " after the spectral type. These red-only spectra have larger uncertainties in the spectral type and extinction measurements. For unresolved multiple stars systems (e.g., TWA 4 AabBab, GG Tau Aab, GG Tau Bab, LkCa $3 \mathrm{AabBab}$, etc.), the spectral type and stellar properties are global measurements of the entire system.

The mass and age estimates are obtained by comparing the photosphere temperature and luminosity to the Baraffe et al. (2003) pre-main-sequence tracks for masses $<0.2 M_{\odot}$, Tognelli et al. (2011) tracks for masses $>0.4 M_{\odot}$, and interpolated between those models for $0.2-0.4 M_{\odot}$. Multiplicity is not accounted for in these mass and age estimates. Severely underluminous stars located below the main sequence have no age listed and a mass (listed in parenthesis) assessed by assuming a $3 \mathrm{Myr}$.

For the 59 stars observed on multiple nights, in Table 14 the veiling and flux at $7510 \AA$ are averages, and the luminosity is calculated from the average flux. These values are each listed independently for each night in the electronic Table. The SpT and, when possible, the extinction are the average of those measured for each spectrum. In several cases, the listed extinctions are different, indicating that no one extinction could accurately explain all spectra from the object. The observed flux at $7510 \AA$ and consequent photospheric luminosity show variability, some of which is attributed to uncertainties in the absolute flux calibration.

Stars with heavy veiling and no spectral type are listed as continuum (c) stars. For these stars, extinction is calculated by assuming the continuum is flat. The listed $F_{7510}$ corresponds to the extinction-corrected flux rather than the photospheric flux, and is listed in parenthesis. In less extreme cases of heavily veiled stars, the spectral type may be estimated and is listed with a "c" following the spectral type.

Spectral types of $\mathrm{M}$ dwarfs are listed to 0.1 subclass, although our internal precision is $\sim 0.2-0.3$ subclasses. Larger differences are likely when comparing spectral types to other studies. Extinctions of stars later than $\mathrm{K} 0$ were measured against our spectral type grid and are listed to $0.05 \mathrm{mag}$ in $A_{V}$. Our extinctions are accurate to $\sim 0.2 \mathrm{mag}$ for stars with little or no veiling.

The extinction measurements assume an extinction law based on a total-to-selective extinction of $R_{V}=3.1$ for most targets. Targets with large extinctions $\left(A_{V}>5\right)$ typically required higher $R_{V}$, indicative of larger grains. V892 Tau could only be fit with an extinction law using $R_{V}>5$ and is assumed to be $R_{V}=5.5$. DoAr 21 and SR 21 (a star with a transition disk) required fits with extinction laws using $R_{V}=4$. For IRS 48, we assumed $R_{V}=5.5$ because of the high extinction.

When possible, we rely on parallax distances: $120 \mathrm{pc}$ for Ophiucus (Loinard et al. 2008), 131 pc for stars near the Lynds 1495 complex in Taurus (Torres et al. 2012), 147 pc for stars near T Tau (Loinard et al. 2007), 161 pc for the stars near the HP Tau complex in Taurus (Torres et al. 2009), 140 pc for all other Taurus objects, and 416 pc for Orion (Menten et al. 2007; Kim et al. 2008). Distances for TWA members are listed in Table 12. We also use $150 \mathrm{pc}$ for Lupus 1 and $200 \mathrm{pc}$ for Lupus 3 (Comeron 2008), 130 pc for CrA (Neuhäuser \& Forbrich 2008), 145 pc for Upper Sco OB Association (Preibisch \& Mamajek 2008), 275 pc for MBM 12 (Luhman 2001), and 200 pc for AT Pyx in the Gum Nebula (Kim et al. 2005).

The distance to RR Tau is not well constrained and is left blank here. The commonly cited distance of $800 \mathrm{pc}$ has often been credited to several more recent publications but was actually calculated by Herbig (1960). The distance to RR Tau was assumed to equal to the distance to the A6 star BD+26 887, located $3^{\prime}$ away. The distance to BD+26 887 was then calculated by comparing its magnitude to that of a main sequence B8 star, based on the spectral type at the time. Hernandez et al. (2004) later adjusted the distance of BD+26 887, but not RR Tau, to $2 \mathrm{kpc}$ based on rough proximity to molecular clouds with distances inferred by (Kawamura et al. 1998). If we assume that AB Aur and RR Tau have the same luminosity, RR Tau would be located at $\sim 670 \mathrm{pc}$. On the other hand, Slesnick et al. (2006) identified pre-main-sequence stars $3^{\circ}$ to the south of RR Tau (RR Tau was not covered in their survey) that have brightnesses consistent with the $\sim 140$ pc distance to Taurus.

\section{REFERENCES}

Alecian, E., Wade, G. A., Catala, C., et al. 2013, MNRAS, 429, 1001 Alencar, S. H. P., \& Batalha, C. 2002, ApJ, 571, 378

Alencar, S. H. P., Teixeira, P. S., Guimaraes, M. M., et al. 2010, A\&A, 519, 88 Allard, F., \& Hauschildt, P. H. 1995, ApJ, 445, 433

Allard, F., Homeier, D., \& Freytag, B. 2012, RSPTA, 370, 2765

Allen, L. E., \& Strom, K. M. 1995, AJ, 109, 1379

Allers, K. N., \& Liu, M. C. 2013, ApJ, 772, 79

Andrews, S. M., Rosenfeld, K. A., Kraus, A. L., \& Wilner, D. J. 2013, ApJ, 771,129

Ardila, D., Martin, E. L., \& Basri, G. 2000, AJ, 120, 479

Bacciotti, F., Mundt, R., Ray, T. P., et al. 2000, ApJL, 537, L49

Baraffe, I., \& Chabrier, G. 2010, A\&A, 521, A44

Baraffe, I., Chabrier, G., Allard, F., \& Hauschlidt, P. H. 1998, A\&A, 337, 403

Baraffe, I., Chabrier, G., Barman, T. S., Allard, F., \& Hauschildt, P. H. 2003, A\&A, 402, 701 
Baraffe, I., Vorobyov, E., \& Chabrier, G. 2012, ApJ, 756, 118

Basri, G., \& Batalha, C. 1990, ApJ, 363, 654

Basri, G., \& Bertout, C. 1989, ApJ, 341, 340

Bell, C. P. M., Naylor, T., Mayne, N. J., Jeffries, R. D., \& Littlefair, S. P. 2013, MNRAS, 434, 806

Beristain, G., Edwards, S., \& Kwan, J. 1998, ApJ, 499, 828

Bertout, C., Basri, G., \& Bouvier, J. 1988, ApJ, 330, 350

Bessell, M. S. 1979, PASP, 91, 589

Bessell, M. S. 1991, AJ, 101, 662

Biazzo, K., Alcala, J. M., Covino, E., et al. 2012, A\&A, 547, 104

Biazzo, K., Randich, S., Palla, F., \& Briceno, C. 2011, A\&A, 530, 19

Biller, B. A., \& Close, L. M. 2007, ApJL, 669, L41

Boden, A. F., Sargent, A. I., Akeson, R. L., et al. 2005, ApJ, 635, 442

Bonnefoy, M., Chauvin, G., Dumas, C., et al. 2009, A\&A, 506, 799

Bouvier, J., Grankin, K., Ellerbroek, L. E., Bouy, H., \& Barrado, D. 2013, A\&A, 557,77

Brandeker, A., Jayawardhana, R., \& Najita, J. 2003, AJ, 126, 2009

Briceno, C., Luhman, K. L., Hartmann, L., Stauffer, J. R., \& Kirkpatrick, J. D 2002, ApJ, 580, 317

Brown, J. M., Pontoppidan, K. M., van Dishoeck, E. F., et al. 2013, ApJ, 770, 94

Calvet, N., D’Alessio, P., Watson, D. M., et al. 2005, ApJL, 630, L185

Calvet, N., \& Gullbring, E. 1998, ApJ, 509, 802

Calvet, N., \& Hartmann, L. 1992, ApJ, 386, 239

Calvet, N., Muzerolle, J., Briceno, C., et al. 2004, AJ, 128, 1294

Cannon, A. J. 1912, AnHar, 56, 65

Cardelli, J. A., Clayton, G. C., \& Mathis, J. S. 1989, ApJ, 345, 245

Casagrande, L., Flynn, C., \& Bessell, M. 2008, MNRAS, 389, 585

Chapman, N. L., Lai, S.-P., Mundy, L.-G., et al. 2007, ApJ, 667, 288

Chapman, N. L., Mundy, L. G., Lai, S.-P., \& Evans, N. J. 2009, ApJ, 690, 496

Chauvin, G., Menard, F., Fusco, T., et al. 2002, A\&A, 394, 949

Cieza, L. A., Olofsson, J., Harvey, P. M., et al. 2013, ApJ, 762, 100

Coffey, D., Bacciotti, F., \& Podio, L. 2008, ApJ, 689, 1112

Cohen, M., \& Kuhi, L. 1979, ApJS, 41, 743

Comeron, F. 2008, in The Lupus Clouds, Handbook of Star Forming Regions, Vol. II, ed. B. Reipurth (San Francisco, CA: ASP), 295

Correia, S., Zinnecker, H., Ratzka, Th., \& Sterzik, M. F. 2006, A\&A, 459, 909

Covey, K. R., Ivezic, Z., Schlegel, D., et al. 2007, AJ, 134, 2398

Covey, K. R., Hillenbrand, L. A., Miller, A. A., et al. 2011, AJ, 141, 40

da Rio, N., Robberto, M., Soderblom, D. R., et al. 2010, ApJ, 722, 1092

Debes, J. H., Jang-Condell, H., Weinberger, A. J., Roberge, A., \& Schneider, G. 2013, ApJ, 771, 45

de la Reza, R., Torres, C. A. O., Quast, G., Castillo, B. V., \& Vieira, G. L. 1989, ApJL, 343, L61

Dodin, A. V., \& Lamzin, S. A. 2012, AstL, 38, 649

D’Orazi, V., Biazzo, K., \& Randich, S. 2011, A\&A, 526, A103

D’Orazi, V., Randich, S., Flaccomio, E., et al. 2009, A\&A, 501, 973

Ducourant, C., Teixeira, R., Galli, P. A. B., et al. 2014, A\&A, in press (arXiv:1401.1935)

Edwards, S., Kwan, J., Fischer, W., et al. 2013, ApJ, 778, 148

Eislöffel, J., \& Mundt, R. 1998, AJ, 115, 1554

Findeisen, K., Hillenbrand, L., Ofek, E., et al. 2013, ApJ, 768, 93

Fischer, W., Edwards, S., Hillenbrand, L., \& Kwan, J. 2011, ApJ, 730, 73

Folha, D. F. M., \& Emerson, J. P. 1999, A\&A, 352, 517

Forestini, M. 1994, A\&A, 285, 473

Furlan, E., Hartmann, L., Calvet, N., et al. 2006, ApJS, 165, 568

Furlan, E., Luhman, K. L., Espaillat, C., et al. 2011, ApJS, 195, 3

Gahm, G. F., Walter, F. M., Stempels, H. C., Petrov, P. P., \& Herczeg, G. J. 2008, A\&A, 482, L35

Gizis, J. E. 2002, ApJ, 575, 484

Gizis, J. E., Jao, W.-C., Subasavage, J. P., \& Henry, T. J. 2007, ApJL, 669, L45

Gray, D. F. 2005, The Observation and Analysis of Stellar Photospheres (3rd ed.; Cambridge: Cambridge Univ. Press)

Gullbring, E., Calvet, N., Muzerolle, J., \& Hartmann, L. 2000, ApJ, 544, 927

Gullbring, E., Hartmann, L., Briceno, C., \& Calvet, N. 1998, ApJ, 492, 323

Hamann, F., \& Persson, S. E. 1992, ApJS, 82, 247

Hartigan, P., Edwards, S., \& Ghandour, L. 1995, ApJ, 452, 736

Hartigan, P., \& Kenyon, S. J. 2003, ApJ, 583, 334

Hartigan, P., Kenyon, S. J., Hartmann, L., et al. 1991, ApJ, 382, 617

Hartigan, P., Strom, K. M., \& Strom, S. E. 1994, ApJ, 427, 961

Hartmann, L., Calvet, N., Gullbring, E., \& D'Alessio, P. 1998, ApJ, 495, 385

Hauschildt, P. H., Allard, F., \& Baron, E. 1999, ApJ, 512, 377

Herbig, G. H. 1960, ApJS, 4, 337

Herbig, G. H., \& Bell, K. R. 1988, Third Catalog of Emission-Line Stars of the Orion Population: 3 (Santa Cruz, CA: Lick Observatory)

Herczeg, G. J., Cruz, K. L., \& Hillenbrand, L. A. 2009, ApJ, 696, 1589

Herczeg, G. J., \& Hillenbrand, L. A. 2008, ApJ, 681, 594
Hernandez, J., Calvet, N., Briceno, C., Hartmann, L., \& Berlind, P. 2004, AJ, 127,1682

Heyer, M. H., \& Graham, J. A. 1989, PASP, 101, 816

Hillenbrand, L. A. 1997, AJ, 113, 1733

Hillenbrand, L. A., Bauermeister, A., \& White, R. J. 2008, in ASP Conf. Ser. 384, 14th Cambridge Workshop on Cool Stars, Stellar Systems, ed. G. van Belle (San Francisco, CA: ASP), 200

Hillenbrand, L. A., Knapp, G. R., Padgett, D. L., Rebull, L. M., \& McGehee, P. M. 2012, AJ, 143, 37

Horne, D., Gibb, E., Rettig, T. W., et al. 2012, ApJ, 754, 64

Indebetouw, R., Mathis, J. S., Babler, B. L., et al. 2005, ApJ, 619, 931

Ingleby, L., Calvet, N., Herczeg, G., et al. 2013, ApJ, 767, 112

Kastner, J. H., Zuckerman, B., \& Bessell, M. 2008, A\&A, 491, 829

Kawamura, A., Onishi, T., Yonekura, Y., et al. 1998, ApJS, 117, 387

Kenyon, S. J., Gomez, M., \& Whitney, B. A. 2008, in Handbook of Star Forming Regions, Vol. 1: The Northern Sky, ed. B. Reipurth (ASP Monograph Publ. Vol. 4; San Francisco, CA: ASP), 405

Kenyon, S. J., \& Hartmann, L. 1995, ApJS, 101, 117

Kim, J. S., Walter, F. M., \& Wolk, S. J. 2005, AJ, 129, 1564

Kim, M. K., Hirota, T., Honma, M., et al. 2008, PASJ, 60, 991

Kirkpatrick, J. D., Henry, T. J., \& McCarthy, D. W. 1991, ApJS, 77, 417

Kirkpatrick, J. D., Kelly, D. M., Rieke, G. H., et al. 1993, ApJ, 402, 643

Konopacky, Q. M., Ghez, A. M., Duchene, G., McCabe, C., \& Macintosh, B. A. 2007, AJ, 133, 2008

Kraus, A. L., \& Hillenbrand, L. A. 2009, ApJ, 704, 531

Kraus, A. L., Ireland, M. J., Hillenbrand, L. A., \& Martinache, F. 2012, ApJ, 745,19

Kraus, A. L., Ireland, M. J., Martinache, F., \& Hillenbrand, L. A. 2011, ApJ, 731,8

Laskar, T., Soderblom, D. R., Valenti, J. A., \& Stauffer, J. R. 2009, ApJ, 698,660

Leggett, S. K. 1992, ApJS, 82, 351

Loinard, L., Torres, R. M., Mioduszewski, A. J., \& Rodriguez, L. F. 2008, ApJL, 675, L29

Loinard, L., Torres, R. M., Mioduszewski, A. J., et al. 2007, ApJ, 671, 546

Looper, D. L., Bochanski, J. J., et al. 2010a, ApJL, 714, L1486

Looper, D. L., Mohanty, S., Bochanski, J. J., et al. 2010b, ApJL, 714, L45

Luhman, K. L. 1999, ApJ, 525, 466

Luhman, K. L. 2001, ApJ, 560, 287

Luhman, K. L. 2004, ApJ, 617, 1216

Luhman, K. L. 2006, ApJ, 645, 676

Luhman, K. L., Allen, P. R., Espaillat, C., Hartmann, L., \& Calvet, N. 2010, ApJS, 186, 111

Luhman, K. L., Mamajek, E. E., Allen, P. R., \& Cruz, K. L. 2009, ApJ, 703, 399

Luhman, K. L., Stauffer, J. R., Muench, A. A., et al. 2003, ApJ, 593, 1093

Magnani, L., Blitz, L., \& Mundy, L. 1985, ApJ, 295, 402

Malo, L., Doyon, R., Lafreniere, D., et al. 2013, ApJ, 762, 88

Mamajek, E. E. 2005, ApJ, 634, 1385

Manara, C. F., Beccari, G., Da Rio, N., et al. 2013a, A\&A, 558, 114

Manara, C. F., Robberto, M., Da Rio, N., et al. 2012, ApJ, 755, 154

Manara, C. F., Testi, L., Rigliaco, E., et al. 2013b, A\&A, 551, 107

McCarthy, J. K., Cohen, J. G., Butcher, B., et al. 1998, Proc. SPIE, 3355, 81

McClure, M. K., Calvet, N., Espaillat, C., et al. 2013, ApJ, 769, 73

McJunkin, M., France, K., Schneider, G. J., et al. 2014, ApJ, 780, 150

Meeus, G., Juhasz, A., Henning, Th., et al. 2009, A\&A, 497, 379

Menten, K. M., Reid, M. J., Forbrich, J., \& Brunthaler, A. 2007, A\&A, 474, 515

Meyer, M. R., Calvet, N., \& Hillenbrand, L. A. 1997, AJ, 114, 288

Mooley, K., Hillenbrand, L., Rebull, L., Padgett, D., \& Knapp, G. 2013, ApJ, 771,110

Mora, A., Merin, B., Solano, E., et al. 2001, A\&A, 378, 116

Muzerolle, J., Calvet, N., Briceno, C., Hartmann, L., \& Hillenbrand, L. 2000, ApJL, 535, L47

Naylor, T. 2009, MNRAS, 399, 432

Neuhä'user, R., \& Forbrich, J. 2008, in The Corona Australis Star Forming Region, Handbook of Star Forming Regions, Vol. II, ed. B. Reipurth (San Francisco, CA: ASP), 735

Nguyen, D. C., Brandeker, A., van Kerkwijk, M. H., \& Jayawardhana, R. 2012, ApJ, 745, 119

Oke, J. B. 1990, AJ, 99, 1621

Oke, J. B., Cohen, J. G., Carr, M., et al. 1995, PASP, 107, 375

Oke, J. B., \& Gunn, J. E. 1982, PASP, 94, 586

Oliveira, I., Merin, B., Pontoppidan, K. M., \& van Dishoeck, E. F. 2013, ApJ, 762,128

Padgett, D. L. 1996, ApJ, 471, 847

Pecaut, M. J., \& Mamajek, E. E. 2013, ApJS, 208, 9

Petrov, P. P., Gahm, G. F., Stempels, H. C., Walter, F. M., \& Artemenko, S. A. 2011, A\&A, 535, 6 
Phillips, A. C., Miller, J., Cowley, D., \& Wallace, V. 2006, Proc. SPIE, 6269, 56 Pickles, A. J. 1998, PASP, 110, 863

Prato, L., Ghez, A. M., Pina, R. K., et al. 2001, ApJ, 549, 590

Preibisch, T. 2012, RAA, 12, 1

Preibisch, T., Brown, A., Bridges, T., Guenther, E., \& Zinnecker, H. 2002, AJ, 124,404

Preibisch, T., \& Mamajek, E. 2008, in Handbook of Star Forming Regions, Vol. 2, The Southern Sky, ed. B. Reipurth (ASP Monograph Publications, Vol. 5; San Francisco, CA: ASP), 235

Rajpurohit, A. S., Reylé, C., Allard, F., et al. 2013, A\&A, 556, 15

Rebull, L. M., Padgett, D. L., McCabe, C.-E., et al. 2010, ApJS, 186, 259

Reggiani, M., Robberto, M., Da Rio, N., et al. 2011, A\&A, 534, 83

Reid, I. N., Hawley, S. L., \& Gizis, J. E. 1995, AJ, 110, 1838

Rich, R. M. 1988, AJ, 95, 828

Riddick, F. C., Roche, P. F., \& Lucas, P. W. 2007, MNRAS, 381, 1067

Santos, N. C., Melo, C., James, D. J., et al. 2008, A\&A, 480, 889

Schmidt-Kaler, T. 1982, in Landolt-Börnstein, Group VI, Vol. 2, ed. K.-H. Hellwege (Berlin: Springer), 454

Schneider, A., Melis, C., \& Song, I. 2012, ApJ, 754, 39

Shkolnik, E. L., Liu, M. C., Reid, I. N., Dupuy, T., \& Weinberger, A. J. 2011, ApJ, 727, 6

Sicilia-Aguilar, A., Henning, T., \& Hartmann, L. W. 2010, ApJ, 710, 597

Skrutskie, M. F., Cutri, R. M., Stiening, R., et al. 2006, AJ, 131, 1163

Slesnick, C. L., Carpenter, J. M., Hillenbrand, L. A., \& Mamajek, E. E. 2006, AJ, 132,2665

Stassun, K. G., Mathieu, R. D., \& Valenti, J. A. 2007, ApJ, 664, 1154

Stelzer, B., Frasca, A., Alcala, J. M., et al. 2013, A\&A, 558, 141
Straizys, V. 1992, Multicolor Stellar Photometry (Tucson, AZ: Pachart) Taguchi, Y., Itoh, Y., \& Mukai, T. 2009, PASJ, 61, 251

Teixeira, R., Ducuourant, C., Chauvin, G., et al. 2008, A\&A, 489, 825

Tognelli, E., Prada Moroni, P. G., \& Degl'Innocenti, S. 2011, A\&A, 533, 109

Torres, G., Ruiz-Rodriguez, D., Badenas, M., et al. 2013, ApJ, 773, 40

Torres, R. M., Loinard, L., Mioduszewski, A. J., \& Rodriguez, L. F. 2009, ApJ, 698, 242

Torres, R. M., Loinard, L., Mioduszewski, A. J., et al. 2012, ApJ, 747, 18

Vacca, W. D., \& Sandell, G. 2011, ApJ, 732, 8

van Leeuwen, F. 2007, A\&A, 414, 653

Valenti, J. A., Basri, G., \& Johns, C. M. 1993, AJ, 106, 2024

Valenti, J. A., \& Fischer, D. A. 2005, ApJS, 159, 141

von Fraunhofer, J. 1814, Denkschriften der Kniglichen Akademie der Wissenschaften zu München, 5, 193

Webb, R. A., Zuckerman, B., Platais, I., et al. 1999, ApJL, 512, L63

Weinberger, A. J., Anglada-Escudé, G., \& Boss, A. P. 2013, ApJ, 762, 118

Weingartner, J. C., \& Draine, B. T. 2001, ApJ, 548, 296

Weintraub, D. A., Saumon, D., Kastner, J. H., \& Forveille, T. 2000, ApJ, 530,867

White, R. J., Gabor, J. M., \& Hillenbrand, L. A. 2007, ApJ, 133, 2524

White, R. J., \& Ghez, A. M. 2001, ApJ, 556, 265

White, R. J., \& Hillenbrand, L. A. 2004, ApJ, 616, 998

Worthey, G., Faber, S. M., Gonzalez, J., \& Burstein, D. 1994, ApJ, 94, 687

Yang, H., Johns-Krull, C. M., \& Valenti, J. A. 2005, ApJ, 635, 466

Zuckerman, B., \& Song, I. 2004, ARA\&A, 42, 685

Zuckerman, B., Webb, R. A., Schwartz, M., \& Becklin, E. E. 2001, ApJL, 549, L233 